JOURNAL OF ETHNOBIOLOGY

AND ETHNOMEDICINE

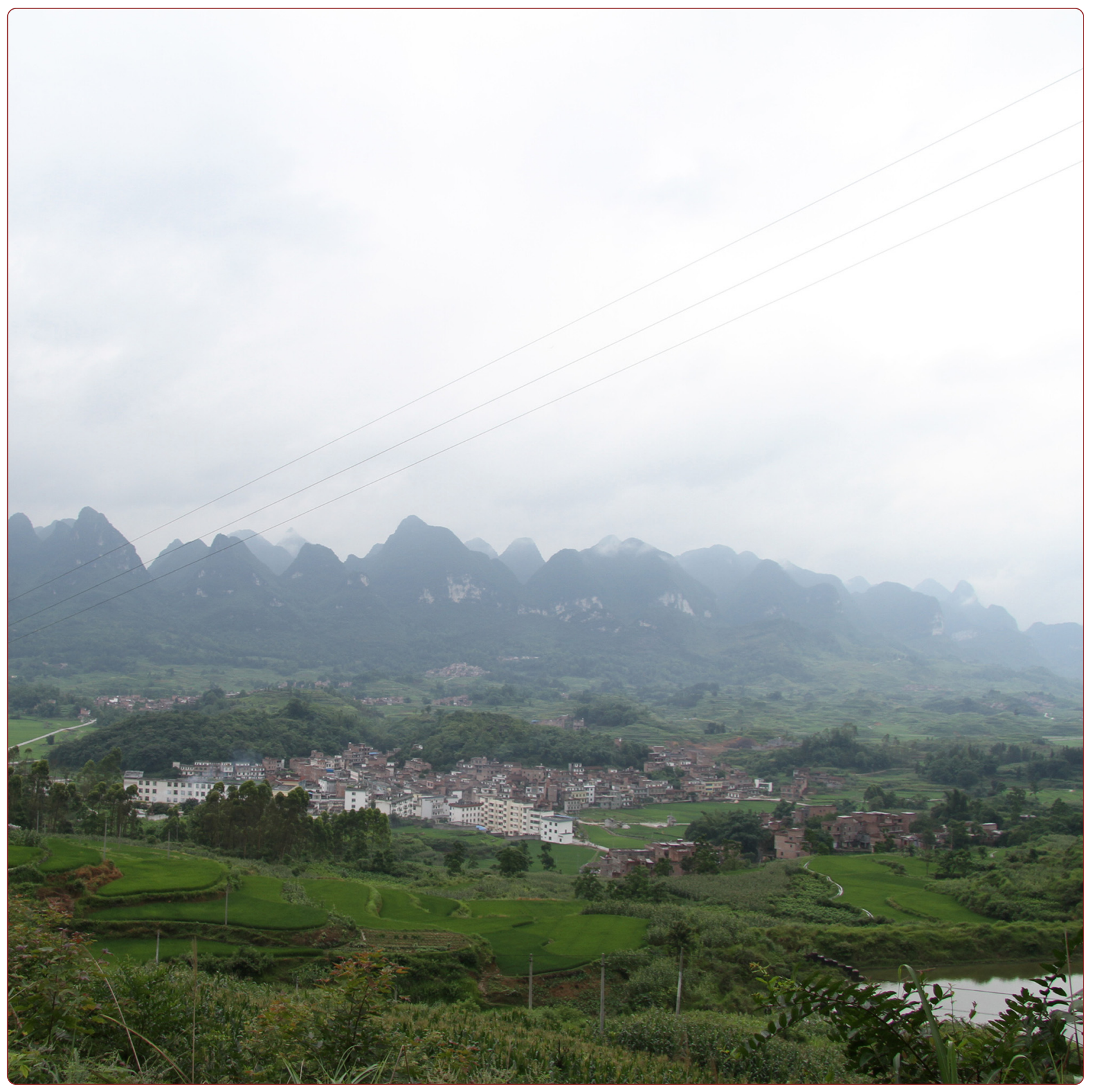

\title{
Ethnobotanical study on medicinal plants used by Maonan people in China
}

Hong et al. 


\title{
Ethnobotanical study on medicinal plants used by Maonan people in China
}

\author{
Liya Hong ${ }^{1}$, Zhiyong Guo ${ }^{1}$, Kunhui Huang ${ }^{1}$, Shanjun Wei ${ }^{1}$, Bo Liu', Shaowu Meng² and Chunlin Long ${ }^{1,3^{*}}$
}

\begin{abstract}
Background: This paper is based on an ethnobotanical investigation that focused on the traditional medicinal plants used by local Maonan people to treat human diseases in Maonan concentration regions. The Maonan people have relied on traditional medicine since ancient times, especially medicinal plants. The aim of this study is to document medicinal plants used by the Maonans and to report the status of medicinal plants and associated traditional knowledge.

Methods: Ethnobotanical data were collected from June 2012 to September 2014 in Huanjiang Maonan Autonomous County, northern Guangxi, southwest China. In total, 118 knowledgeable informants were interviewed. Following statistically sampling method, eighteen villages from 5 townships were selected to conduct field investigations. Information was collected through the approache of participatory observation, semi-structured interviews, ranking exercises, key informant interviews, focus group discussions, and participatory rural appraisals.
\end{abstract}

Results: A total of 368 medicinal plant species were investigated and documented together with their medicinal uses by the Maonans, most of which were obtained from the wild ecosystems. The plants were used to treat 95 human diseases. Grinding was a widely used method to prepare traditional herbal medicines. There were significant relationships between gender and age, and between gender and informants' knowledge of medicinal plant use. Deforestation for agricultural purposes was identified as the most destructive factor of medicinal plants, followed by drought and over-harvest.

Conclusions: The species diversity of medicinal plants used by the Maonans in the study area was very rich. Medicinal plants played a significant role in healing various human disorders in the Maonan communities. However, the conflicts between traditional inheriting system and recent socio-economic changes (and other factors) resulted in the reduction or loss of both medicinal plants and associated indigenous knowledge. Thus, conservation efforts and policies, and innovation of inheriting system are necessary for protecting the medicinal plants and associated indigenous knowledge. Awareness is also needed to be raised among local Maonans focusing on sustainable utilization and management of both medicinal plants and traditional knowledge.

Keywords: Medicinal plants, Traditional knowledge, The Maonans, Ethnomedicine, Huanjiang county

\section{Background}

Traditional medicine is used to maintain people's health, as well as to prevent, diagnose, improve or treat physical and mental illnesses all over the world [1,2]. Medicinal plants are believed to be with healing powers, and people have used them for many centuries. Aimed to

\footnotetext{
* Correspondence: long@mail.kib.ac.cn

'College of Life and Environmental Sciences, Minzu University of China,

Beijing 100081, People's Republic of China

${ }^{3}$ Kunming Institute of Botany, Chinese Academy of Sciences, Kunming 650201, People's Republic of China

Full list of author information is available at the end of the article
}

modern drug discovery, traditional medicinal plants have been studied and developed which is followed the ethnobotanical lead of indigenous cures used by traditional medical systems [3-5]. Traditional medicinal knowledge, especially using medicinal plants in the developing countries, has been in existence and use, and has been a part of therapeutic practices [6]. Therefore, the investigation of plants and their uses (especially medicinal purposes) is one of the most primary human concerns and has been practiced in the world [7-12].

\section{() Biomed Central}

(c) 2015 Hong et al.; licensee BioMed Central. This is an Open Access article distributed under the terms of the Creative Commons Attribution License (http://creativecommons.org/licenses/by/4.0), which permits unrestricted use, distribution, and reproduction in any medium, provided the original work is properly credited. The Creative Commons Public Domain Dedication waiver (http://creativecommons.org/publicdomain/zero/1.0/) applies to the data made available in this article, unless otherwise stated. 
The traditional use of medicinal plants in China is widely accepted. The population of 55 minorities is 11.2 millions occupying $8 \%$ of China's population, and these minorities distribute in $65 \%$ of the country's territory. Each minority has its own medicinal characteristic, and has various experiences of medicinal knowledge [13]. Traditional medicinal plants play an important role of protecting people's lives and health in minority regions, especially in remote and poor area $[14,15]$. Because of unique natural conditions and customs in the ethnic minority areas, long-term practices of using medicinal plants have formed various systems of treating diseases [16-18]. For example, Tibetan medicine is famous for treating digestive disorders, rheumatic diseases and wounds [19,20]. The Mongolians have a long history of horse riding, and their medicine is effective to deal with bone fracture and brain concussion. Yao medicine has special advantages in cancers and skin problems [21].

North Guangxi has been recognized as a rich biodiversity and world-famous karst area. With the elevation between $700-1500 \mathrm{~m}$, it is obviously affected by plateau terrain and subtropical monsoon climate. Thus, the temperature difference of four seasons is small but the vertical climate changes significantly. There are more than 10 ethnic groups living in north Guangxi and formed colorful ethnic characteristic. As one of the indigenous minorities, Maonan is mainly living in Huanjiang Maonan Autonomous County, Guangxi Zhuang Autonomous Region, southwest China. The exceptional altitudinal range, topography and climatic variability in this region have fostered a center of plant species endemism. Here the majority of Maonan people rely on medicinal plants for self-medication. The Maonan medicine has made a great contribution to protect the health of local people. This is due to free access to medicinal herbs, cultural traditions and high cost of hospital treatments in the town nearby. Local people widely utilize endemic species, and they have developed their own traditional medicinal knowledge. Without writing language, Maonan people pass on their indigenous knowledge from generation to generation orally. Nowadays, the Maonan children spend most of their time in schools, where they are taught in Han language. This decreases their chances to learn about the uses of the medicinal plants from the old people. Therefore, important information about medicinal plants is easily lost in the transfer process of indigenous knowledge. With the impact of increasing modern health facilities and modern civilization in Maonan area, indigenous knowledge is depleting rapidly. Although a number of ethnobotanical documentations about several ethnic groups have been published during the past decades in China, few field ethnobotanical studies have been conducted in Maonan society. It is therefore necessary to carry out a survey to document the medicinal plants and associated indigenous knowledge in Maonan region.

Thus, the purposes of the present work were as follows: (i) to document and analyze the knowledge and use of medicinal plants by Maonan people at the study area; (ii) to circulate the results within the scientific community in order to open a door for research in other disciplines; (iii) to document the medicinal plants that could be valuable in future's phytochemical and pharmacological discoveries, and (iv) to contribute to the knowledge and conservational possibilities of plant biodiversity, bearing in mind that biological diversity is also related to the use and applications of natural resources.

\section{Materials and methods}

\section{Study area and the people}

The study area covered 18 villages of Huanjiang County (the only Maonan autonomous County in China) in the northern part of Guangxi Zhuang Autonomous Region, southwest China (Figure 1). The villages are located in 5 townships, which were selected based on Maonan traditional settlements, namely: Chengnan, Chengbei, Luoyang, Shuiyuan, Shangnan, Youdong, Mulun, Xia'nan, Pochuan, Fengyi, Zhongnan, Tangba, Xiatang, Yuhuan, Caimen, Guzhou, Xiyuan, and Jingyang villages. Huanjiang County is situated in the subtropical zone, located between $24^{\circ} 83^{\prime}$ and $25^{\circ} 06^{\prime}$ east longitude and between $107^{\circ} 92^{\prime}$ and $108^{\circ}$ $26^{\prime}$ north latitude, with the annual average temperature of $20^{\circ} \mathrm{C}$ and annual rain fall of $1500 \mathrm{~mm}$. The vegetation of the county belongs to the subtropical evergreen montane forest. It is humid in summer and relatively dry in winter. The most Maonan villages are seated on the small strips of flat land or slopes in the rocky mountainous area at 5001000 meters above sea level. The sinkholes and underground caverns in the area have well developed because of karst landform. Despite abundant rainfall, there are no big rivers but only a small number of streams. Water shortage has been a major obstacle to economic and social development in the Maonan areas.

The Maonan minority, with a total population of about 107,200 , is one of the 55 officially recognized ethnic groups in China. With no written language [22], the Maonans' stories and traditions are remembered and passed down orally from generation to generation, but these are becoming less and less. The Maonan language belongs to the Dong-Shui branch of the Zhuang-Dong language group in the Chinese-Tibetan language family. The Maonan language is widely spoken in Maonan communities. Almost all of the Maonans know both Han and Zhuang languages, because they need to communicate with the Zhuang and Han people, the majorities in Guangxi. About $60 \%$ of the Maonan people live in Huanjiang County, which is the only Maonan autonomous county in China. The Maonans are polytheistic, 


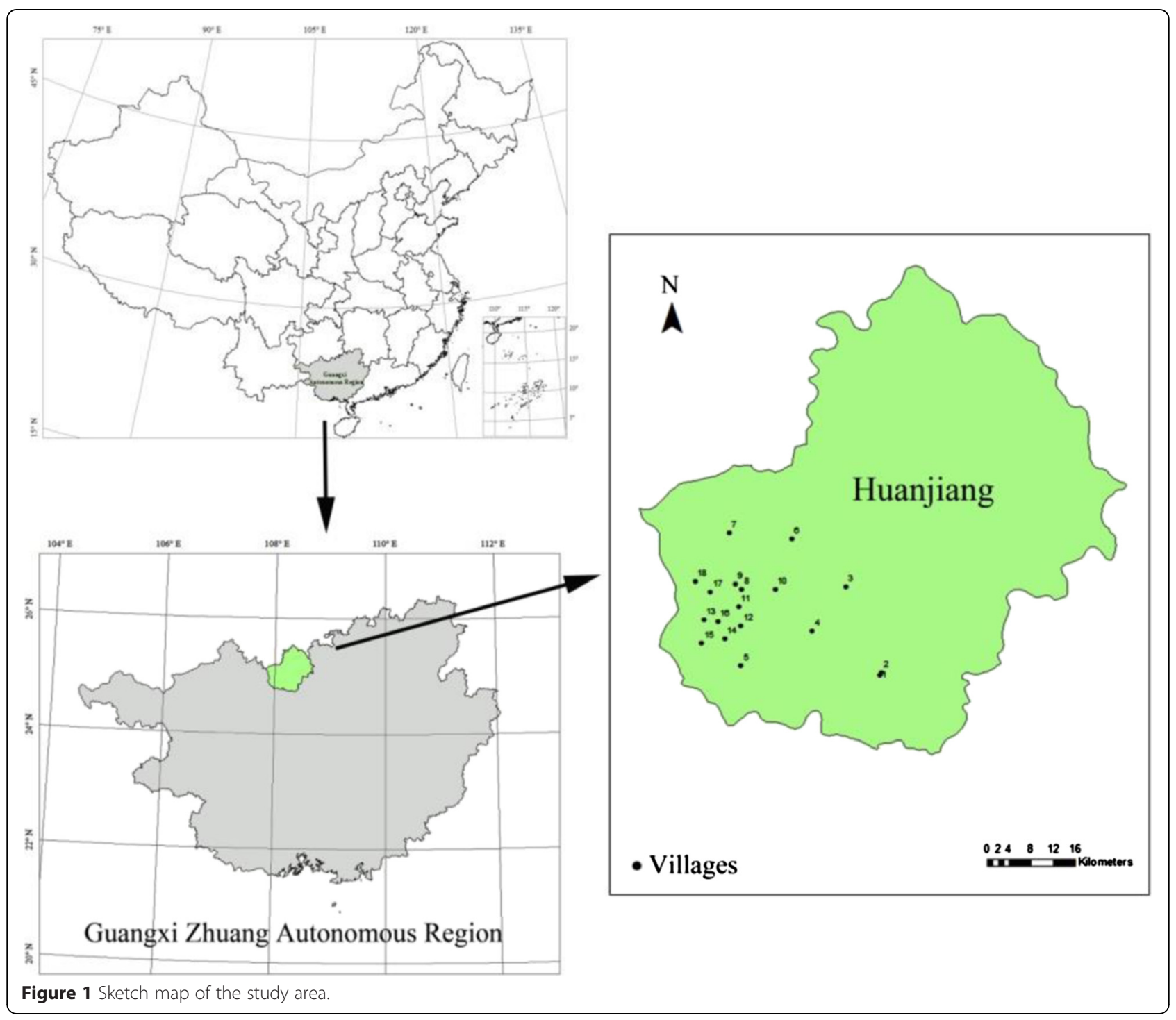

and they pay homage to dozens of deities or immortals on various occasions. These icons include figures from myths, legends, celebrities of historical events, divinities from Taoism or Buddhism, ancestors of the family and so on [22]. Due to remote mountainous regions and poor economic environment, traditional remedies of medicinal plants are the most important and sometimes the only source of therapeutics in the Maonan villages. The long utilization history and traditional knowledge of medicinal plants had supported their livelihoods. The Maonan healers and farmers have developed their own ethnomedicinal knowledge.

\section{Field works and ethnobotanical data collection}

A total of 118 (106 males and 12 females) informants were interviewed in the study area, in which 80 were selected using snowball technique and 38 key informants were selected purposively and systematically based on the recommendations of knowledgeable elders, local authorities and development agents. All of the informants were local inhabitants aged between 21 and 85 years. Local Maonan healers were surely identified as key informants, because they were important custodians and participants of indigenous knowledge of medicinal plants. Interestingly, all these traditional healers were males. A few women were also interviewed to examine their medicinal knowledge and opinions.

Ethnobotanical investigations were carried out to collect data on medicinal plants used to treat human ailments following standard methods in Maonan area. The methodological approaches were semi-structured interviews, field observations, group discussions and guided field walks. The data were collected from June 2012 to September 2014. Interviews and discussions were undertaken based on a checklist of questions prepared in Chinese and translated into Maonan language. Information was carefully 
recorded during interviews with each informant. Field observations were performed with traditional healers guided on the morphological features and habitats of each medicinal plant species. Voucher specimens of cited medicinal plants were collected and their local identity was reconfirmed by other informants. The information obtained was cross-checked with the other informants. The information such as the local name, habit, wild/ cultivated, availability of medicinal plants, need of conservation and efforts made by inhabitants, and traditional medicinal uses of plants were recorded. Group discussions were conducted on multipurpose, conservation, threats of the medicinal plants, and transferability of knowledge with the healers and local people in the villages. Also, the key informants were selected for preference ranking exercise.

\section{Specimen collection and identification}

The listed medicinal plants were collected from field and gardens, and the habits of these plants were recorded. The voucher specimens were made and deposited in the Herbarium, College of Life and Environmental Sciences, Minzu University of China, Beijing, China, for future references. The botanical identities of collected specimens were confirmed by the authors and other taxonomists at Minzu University of China. Plant names were checked with Flora of China and botanical websites (e.g. http://www.tropicos.org/).

\section{Data analysis}

The data were summarized using Microsoft Office Excel sheet. Descriptive statistical methods were applied to analyze and summarize the ethnobotanical data such as frequency and percentage.

Preference ranking exercise $[23,24]$ was conducted by 8 key informants on 7 medicinal plants used to treat traumatic injury in the study area. The highest number of medicinal plants was prescribed by informants to fight traumatic injury. The plants in this exercise were shortlisted by the key informants, and then their importance to manage traumatic injury was discussed. The plants were given to the informants and were ranked based on their efficacy. Medicinal plant that was believed to be the most effective was given the highest value 7 , and the one with the least effectiveness a value of 1 . Rank was determined based on the total score of each species. A total rank of preference exercise was obtained by summing the number of informant given.

The reported ailments were grouped into 21 categories based on the information gathered from the interviewees. Factor of informant consensus $\left(\mathrm{F}_{\mathrm{IC}}\right)$ was calculated for each category to test the agreements of the informants on the reported cures for the group of diseases. The $\mathrm{F}_{\mathrm{IC}}$ was calculated as follows: number of use citations in each category $\left(\mathrm{N}_{\mathrm{ur}}\right)$ minus the number of species used $\left(\mathrm{N}_{\mathrm{t}}\right)$, and divided by the numbers of use citations in each category minus one $[25,26]$. The formula was listed as below:

$$
\mathrm{F}_{\mathrm{IC}}=\left(\mathrm{N}_{\mathrm{ur}}-\mathrm{N}_{\mathrm{t}}\right) /\left(\mathrm{N}_{\mathrm{ur}}-1\right)
$$

\section{Results}

\section{Medicinal plants reported}

The study recorded 368 medicinal plant species (see Table 1). Ethnomedicinal information for each species, including scientific name, Chinese name, local name, family name, life form, habitat, plant parts used, preparation and uses, was listed in Table 1. The species belonged to 295 genera and 115 families were used by Maonan people to treat various human ailments. Among the families that contributed more medicinal species were Asteraceae, represented by 24 species (6.52\%), Fabaceae with 19 (5.16\%) species, and Rosaceae with 16 (4.35\%), while other 292 families contributed 309 (83.97\%) species were mostly represented by 1 or 2 species (Table 2).

The distribution of informants in age, gender and education class was shown in Table 3. The majority of informants interviewed were above 40 years old in this investigation. The male informants were $89.8 \%$ and less educated. There was a significant correlation between the informant age and phytomedicinal knowledge.

\section{Life forms, plant parts used, method of collection and administration}

The result of life form analysis of medicinal plants showed that herbaceous plants constituted the highest proportion represented by $203(55.16 \%)$ species, while there were 67 (18.21\%) shrubs species, 43 (11.68\%) lianas and 41 (11.14\%) tree species (Figure 2).

Informants of the study area used different plant parts for preparation of traditional drugs (e.g. leaves, roots, seeds, barks and fruits). The informants reported that more species (153) of medicinal plants were harvested for their whole plants, and these were followed by roots (83), leaves (45), stems (30), fruits (29), tubers (29), rhizomes (27) and 51 other parts (seed, bark, flower and so on) (Figure 3). The majority of remedies were prepared from fresh materials, and some were prepared from either dried or fresh materials while a few were only used from dried materials.

Of these 368 species of medicinal plants collected from the study area, most of them $(256,67.72 \%)$ were obtained from the wild habitats whereas 54 (14.67\%) were from home gardens, and only 58 (15.76\%) species were from both home gardens and wild habitats (see Table 1). The majority of plants used as medicine were freely harvested by healers from natural environment, 
Table 1 Inventory of Medicinal Plants Traditionally Used by Maonan People

\begin{tabular}{|c|c|c|c|c|c|c|c|c|}
\hline No. & Scientific name & Chinese name & Maonan name & Family & $\begin{array}{l}\text { Life } \\
\text { form }\end{array}$ & Habit & Parts used & Preparation and uses \\
\hline 1 & $\begin{array}{l}\text { Abelmoschus } \\
\text { sagittifolius (Kurz) Merr. }\end{array}$ & Jianyeqiukui箭叶秋葵 & - & Malvaceae & Herb & Wild & Root & Grinding, decoction; Taken orally for furuncle \\
\hline 2 & Abrus cantoniensis Hance & $\begin{array}{l}\text { Guangdong xiangsizi广 } \\
\text { 州相思子 }\end{array}$ & roun $^{2} \mathrm{ra}^{2} \mathrm{təp}^{7}$ & Fabaceae & Shrub & Wild & Whole plant & $\begin{array}{l}\text { Grinding, decoction; Taken orally for acute } \\
\text { and chronic hepatitis, stomachache, rheumatism, } \\
\text { ostealgia, traumatic injury, liver cirrhosis and } \\
\text { common cold }\end{array}$ \\
\hline 3 & $\begin{array}{l}\text { Abutilon indicum } \\
\text { (L.) Sweet }\end{array}$ & Mopancao磨盘草 & ruon $^{2}$ nan $^{3} l u i n^{5}$ & Malvaceae & Herb & Wild & Whole plant & $\begin{array}{l}\text { Boiled with meat; Taken orally soup, treating } \\
\text { for fever due to common cold, bronchitis, } \\
\text { epidemic parotitis and tuberculosis }\end{array}$ \\
\hline 4 & $\begin{array}{l}\text { Acanthopanax gracilistylus } \\
\text { W. W. Smith. }\end{array}$ & Wujia五加 & $m b a^{3} \operatorname{shhi}^{2} a n^{2} l a u^{4}$ & Araliaceae & Shrub & Both & Root, Bark & $\begin{array}{l}\text { Grinding, decoction; Taken orally for rheumatic } \\
\text { arthritis, traumatic injury, carminative, bone } \\
\text { fracture and pain of limbs }\end{array}$ \\
\hline 5 & $\begin{array}{l}\text { Acanthopanax } \\
\text { trifoliatus (L.) Merr. }\end{array}$ & Baile白簕 & $m b a^{3} \operatorname{tshi}^{6} \operatorname{man}^{2} n d i^{5}$ & Araliaceae & Shrub & Both & Stem, Root & $\begin{array}{l}\text { Medicinal liquor for treating rheumatic arthritis, } \\
\text { traumatic injury, waist and legs pain, ostealgia } \\
\text { and sciatica; Pound fresh part applied on the } \\
\text { affected area, treating for eczema, ulcer } \\
\text { and furuncle }\end{array}$ \\
\hline 6 & $\begin{array}{l}\text { Achillea wilsoniana } \\
\text { Heimerl ex Hand. -Mazz. }\end{array}$ & Yunnanshi云南著 & - & Asteraceae & Herb & Wild & Whole plant & $\begin{array}{l}\text { Pound fresh part applied on the affected area, } \\
\text { treating for ulcer }\end{array}$ \\
\hline 7 & $\begin{array}{l}\text { Achyranthes bidentata } \\
\text { Blume }\end{array}$ & Niuxi牛膝 & $m a^{6}$ wei $^{5}$ gou $^{2}$ you $^{1}$ & Amaranthaceae & Herb & Wild & Root & $\begin{array}{l}\text { Grinding and drink with wine for traumatic } \\
\text { injury, removing blood stasis }\end{array}$ \\
\hline 8 & $\begin{array}{l}\text { Aconitum carmichaeli } \\
\text { Debx. }\end{array}$ & Wutou乌头 & $\tan ^{5} \mathrm{gou}^{2} 7 \mathrm{no}^{2}$ & Ranunculaceae & Herb & Both & Tuber & $\begin{array}{l}\text { Grinding, decoction; Taken orally for scrofula, } \\
\text { perineum ache }\end{array}$ \\
\hline 9 & Acorus calamus $\mathrm{L}$. & Shuichangpu水菖蒲 & ba: $\eta^{5} s j \varepsilon m^{2} r \partial m^{3}$ & Acoraceae & Herb & Wild & Root & Powder; Taken orally for diarrhea \\
\hline 10 & Acorus tatarinowii Schott & Shichangpu石菖蒲 & ruon $^{2} j \varepsilon \eta^{3} v u^{2}$ & Acoraceae & Herb & Wild & Rhizome & $\begin{array}{l}\text { Grinding, decoction; Taken orally for epilepsy } \\
\text { and convulsion }\end{array}$ \\
\hline 11 & $\begin{array}{l}\text { Adenophora tetraphylla } \\
\text { (Thunb.) Fisch. }\end{array}$ & Lunyeshashen轮叶沙参 & mua $^{2} \operatorname{tcio}^{3} g 7 a i^{2}$ & Campanulaceae & Herb & Wild & Root & $\begin{array}{l}\text { Grinding, decoction; Taken orally for } \\
\text { complications after measles }\end{array}$ \\
\hline 12 & $\begin{array}{l}\text { Adiantum capillus-junonis } \\
\text { Rupr. }\end{array}$ & $\begin{array}{l}\text { tuanyutiexianjue团羽铁 } \\
\text { 线蕨 }\end{array}$ & $\mathrm{ya}^{2} \mathrm{bou}^{3}$ & Adiantaceae & Herb & Wild & $\begin{array}{l}\text { Whole plant, } \\
\text { Rhizome }\end{array}$ & $\begin{array}{l}\text { Boiled with meat and drunk the soup, treating } \\
\text { for piles }\end{array}$ \\
\hline 13 & Aeginetia indica $\mathrm{L}$. & Yegu野菰 & - & Orobanchaceae & Herb & Wild & Whole plant & $\begin{array}{l}\text { Grinding, decoction; Taken orally for swelling, } \\
\text { clearing away heat and toxic materials }\end{array}$ \\
\hline 14 & Ageratum conyzoides $\mathrm{L}$. & Huoxiangj嚾香薊 & - & Asteraceae & Herb & Wild & Whole plant & $\begin{array}{l}\text { Grinding, decoction; Taken orally for fever due } \\
\text { to common cold, empyrosis and abscess }\end{array}$ \\
\hline 15 & Agrimonia pilosa Ledeb. & Longyacao龙芽草 & ruon $^{2}$ hiu'cia $^{3}$ & Rosaceae & Herb & Wild & Root & $\begin{array}{l}\text { Boiled with meat or wine and drunk the soup, } \\
\text { treating for piles, enteritis, diarrhea, hemafecia, } \\
\text { hematuria }\end{array}$ \\
\hline 16 & $\begin{array}{l}\text { Ainsliaea bonatii } \\
\text { Beauverd }\end{array}$ & $\begin{array}{l}\text { Xinyetu'erfeng心叶兔儿 } \\
\text { 风 }\end{array}$ & $m a^{6} k a^{6} \mathrm{zai}^{2}$ & Asteraceae & Herb & Wild & Whole plant & $\begin{array}{l}\text { Grinding, decoction; Taken orally for cough, } \\
\text { asthma with throat itching }\end{array}$ \\
\hline
\end{tabular}


Table 1 Inventory of Medicinal Plants Traditionally Used by Maonan People (Continued)

\begin{tabular}{|c|c|c|c|c|c|c|c|c|}
\hline 17 & $\begin{array}{l}\text { Akebia quinata (Houtt.) } \\
\text { Decne. }\end{array}$ & Mutong木通 & - & Lardizabalaceae & Liana & Wild & Stem, Root, Fruit & $\begin{array}{l}\text { Grinding, decoction; Taken orally for rheumatism, } \\
\text { diuresis, promoting lactation }\end{array}$ \\
\hline 18 & $\begin{array}{l}\text { Alangium chinense } \\
\text { (Lour.) Harms }\end{array}$ & Bajiaofeng八角枫 & $m e i^{4} d a^{2}$ & Alangiaceae & Tree & Both & Fibrous root & $\begin{array}{l}\text { Grinding, decoction; Taken orally for rheumatic } \\
\text { arthritis, lumbar muscle degeneration, asthma } \\
\text { and bleeding }\end{array}$ \\
\hline 19 & Allium fistulosum L. & Cong葱 & $\operatorname{son}^{3} x_{i e n}^{3}$ nien $^{2}$ & Liliaceae & Herb & Homegarden & Whole plant & $\begin{array}{l}\text { Grinding, decoction; Taken orally for common } \\
\text { cold, pains, rheumatic headache, numbness } \\
\text { of limbs and replenishing the liver }\end{array}$ \\
\hline 20 & Allium sativum $\mathrm{L}$. & Suan蒜 & $k ⿰ \eta^{1} \mathrm{do}^{2}$ & Liliaceae & Herb & Homegarden & Bulb & $\begin{array}{l}\text { Grinding, dispersed in water and drunk for } \\
\text { pertussis cough, enteritis, tuberculosis, poor } \\
\text { appetite, indigestion, diarrhea }\end{array}$ \\
\hline 21 & $\begin{array}{l}\text { Allium tuberosum } \\
\text { Rottl. ex Spreng. }\end{array}$ & Jiu非 & $m b a^{3} k \partial n^{5}$ & Liliaceae & Herb & Homegarden & Whole plant & $\begin{array}{l}\text { Grinding, decoction; Taken orally for toothache, } \\
\text { piles, traumatic injury and insect bite }\end{array}$ \\
\hline 22 & $\begin{array}{l}\text { Alocasia macrorrhizos } \\
\text { (L.) G. Don }\end{array}$ & Reyahaiyu热亚海芋 & - & Araceae & Herb & Wild & Whole plant & $\begin{array}{l}\text { Pound fresh part applied on the affected area, } \\
\text { treating for snake bite and innominate } \\
\text { inflanunatory of unknown origin }\end{array}$ \\
\hline 23 & Alpinia katsumadai Hayata & Caodoukou草豆冦 & - & Zingiberaceae & Herb & Homegarden & Fruit & Grinding, decoction; Taken orally for indigestion \\
\hline 24 & Alpinia oxyphylla Miq. & Yizhi益智 & - & Zingiberaceae & Herb & Wild & Fruit & $\begin{array}{l}\text { Grinding, decoction; Taken orally for warming } \\
\text { the spleen, kidney deficiency, diarrhea and } \\
\text { spermatorrhea }\end{array}$ \\
\hline 25 & $\begin{array}{l}\text { Alternanthera sessilis } \\
\text { (L.) DC. }\end{array}$ & Lianzicao莲子草 & - & Amaranthaceae & Herb & Wild & Whole plant & $\begin{array}{l}\text { Grinding, decoction; Taken orally for reducing } \\
\text { fever and causing diuresis }\end{array}$ \\
\hline 26 & $\begin{array}{l}\text { Amomum tsaoko } \\
\text { Crevost et Lemarie }\end{array}$ & Caoguo草果 & - & Zingiberaceae & Herb & Homegarden & Fruit & $\begin{array}{l}\text { Grinding, decoction; Taken orally for eliminating } \\
\text { phlegm, indigestion, diarrhea and malaria }\end{array}$ \\
\hline 27 & $\begin{array}{l}\text { Amomum villosum } \\
\text { Lour. }\end{array}$ & Sharen砂仁 & - & Zingiberaceae & Herb & Homegarden & Fruit & Grinding, decoction; Taken orally for indigestion \\
\hline 28 & $\begin{array}{l}\text { Andrographis paniculata } \\
\text { (Burm. f.) Nees }\end{array}$ & Chuanxinlian穿心莲 & - & Acanthaceae & Herb & Wild & Whole plant & $\begin{array}{l}\text { Grinding, decoction; Taken orally for clearing } \\
\text { away heat and toxic materials }\end{array}$ \\
\hline 29 & $\begin{array}{l}\text { Androsace umbellata (Lour.) } \\
\text { Merr. }\end{array}$ & Diandimei点地梅 & - & Primulaceae & Herb & Wild & Whole plant & $\begin{array}{l}\text { Grinding, decoction; Taken orally for inflammation } \\
\text { and traumatic injury }\end{array}$ \\
\hline 30 & Anemone hupehensis Lem. & $\begin{array}{l}\text { Dapowanhuahua打破 } \\
\text { 碗花花 }\end{array}$ & $m a^{2} m i n^{5} y \varepsilon^{5}$ & Ranunculaceae & Herb & Wild & Root, Whole plant & $\begin{array}{l}\text { Grinding, decoction; Taken orally for biliary tract } \\
\text { ascariasis }\end{array}$ \\
\hline 31 & Aralia chinensis $\mathrm{L}$. & Songmu淴木 & - & Araliaceae & Tree & Wild & Seed & Grinding, decoction; Taken orally for snake bite \\
\hline 32 & $\begin{array}{l}\text { Aralia undulata } \\
\text { Hand.-Mazz. }\end{array}$ & $\begin{array}{l}\text { Boyuansongmu波缘淴 } \\
\text { 木 }\end{array}$ & $m e i^{5}$ sun $^{4}$ & Araliaceae & Shrub & Wild & Root & $\begin{array}{l}\text { Boiled with meat and drunk the soup, treating } \\
\text { for cough }\end{array}$ \\
\hline 33 & Arctium lappa L. & Niubang牛蒡 & ma: ${ }^{6} \mathrm{ka}: \mathrm{wei}^{5}$ & Asteraceae & Herb & Homegarden & Fruit & $\begin{array}{l}\text { Grinding, decoction; Taken orally for infantile } \\
\text { fever and cough }\end{array}$ \\
\hline 34 & $\begin{array}{l}\text { Ardisia gigantifolia } \\
\text { Stapf }\end{array}$ & Zoumatai走马胎 & ruon $^{2} \operatorname{lon}^{2} \mathrm{mia}^{4}$ & Myrsinaceae & Shrub & Wild & $\begin{array}{l}\text { Rhizome, Whole } \\
\text { plant }\end{array}$ & $\begin{array}{l}\text { Medicinal liquor for treating rheumatism, } \\
\text { rheumatic arthritis, waist and legs pain, } \\
\text { paralysis, hemiplegia and traumatic injury }\end{array}$ \\
\hline
\end{tabular}


Table 1 Inventory of Medicinal Plants Traditionally Used by Maonan People (Continued)

\begin{tabular}{|c|c|c|c|c|c|c|c|c|}
\hline 35 & $\begin{array}{l}\text { Ardisia japonica (Thunb.) } \\
\text { Blume }\end{array}$ & Zijinniu紫金牛 & $w a^{5} t_{6 i \varepsilon m^{2} w e i^{3}}$ & Myrsinaceae & Shrub & Wild & Whole plant & $\begin{array}{l}\text { Grinding, decoction; Taken orally for jaundiced } \\
\text { hepatitis, cough, traumatic injury and preventing } \\
\text { phlegm }\end{array}$ \\
\hline 36 & $\begin{array}{l}\text { Arisaema erubescens (Wall.) } \\
\text { Schott }\end{array}$ & $\begin{array}{l}\text { Yibasannanxing一把伞 } \\
\text { 南星 }\end{array}$ & ma'gəp $^{8}$ tai $^{5}$ & Araceae & Herb & Wild & Tuber & $\begin{array}{l}\text { Pound fresh part applied on the affected area, } \\
\text { treating for snake bite }\end{array}$ \\
\hline 37 & $\begin{array}{l}\text { Arisaema heterophyllum } \\
\text { Blume }\end{array}$ & Tiannanxing天南星 & $j \varepsilon k^{7} k h \omega^{6} d \ni \eta^{2}$ & Araceae & Herb & Wild & Tuber & $\begin{array}{l}\text { Grinding, decoction; Taken orally for traumatic } \\
\text { injury, cough, hypertension, acute inflammation } \\
\text { and abdomen pain }\end{array}$ \\
\hline 38 & $\begin{array}{l}\text { Arisaema rhizomatum } \\
\text { C. E. C. Fischer }\end{array}$ & Xuelijian雪里见 & kyou $^{2} \mathrm{nau}^{4}$ & Araceae & Herb & Wild & Tuber & $\begin{array}{l}\text { Medicinal liquor for treating scrofula and } \\
\text { perineum ache }\end{array}$ \\
\hline 39 & $\begin{array}{l}\text { Aristolochia fangchi } \\
\text { Y. C. Wu ex L. D. Chow } \\
\text { et S. M. Hwang }\end{array}$ & Guangfangji广防己 & ruon $^{2} d a k^{8} \mid o n^{2}$ & Aristolochiaceae & Liana & Wild & Tuber & $\begin{array}{l}\text { Grinding, decoction; Taken orally for acute } \\
\text { nephritis, urinary tract infection, hypertension, } \\
\text { rheumatic heart disease, edema }\end{array}$ \\
\hline 40 & $\begin{array}{l}\text { Aristolochia kwangsiensis } \\
\text { Chun et How ex C. F. Liang }\end{array}$ & $\begin{array}{l}\text { Guangximadouling广西 } \\
\text { 马咸铃 }\end{array}$ & - & Aristolochiaceae & Liana & Wild & Tuber & $\begin{array}{l}\text { Grinding, decoction; Taken orally for snake bite, } \\
\text { stomachache, diarrhea, strep throat, epidemic } \\
\text { parotitis, lymphnoditis }\end{array}$ \\
\hline 41 & $\begin{array}{l}\text { Aristolochia versicolor S. M. } \\
\text { Hwang }\end{array}$ & $\begin{array}{l}\text { Biansemadouling变色 } \\
\text { 马晁铃 }\end{array}$ & - & Aristolochiaceae & Liana & Wild & Whole plant & Grinding, decoction; Taken orally for snake bite \\
\hline 42 & $\begin{array}{l}\text { Armeniaca vulgaris } \\
\text { Lam. }\end{array}$ & Xing杏 & dəク'von ${ }^{5} z a^{2}$ & Rosaceae & Tree & Homegarden & Seed & $\begin{array}{l}\text { Grinding, decoction; Taken orally for chronic } \\
\text { trachitis, cough }\end{array}$ \\
\hline 43 & Artemisia annua L. & Huanghuahao黄花蒿 & ruon $^{2} n n a i^{6} \min ^{3}$ & Asteraceae & Herb & Wild & Whole plant & $\begin{array}{l}\text { Grinding, decoction; Taken orally for malaria, } \\
\text { fever, indigestion, tuberculosis hot flashes } \\
\text { and night sweat; washing for scab, pruritus } \\
\text { and mosquito bite }\end{array}$ \\
\hline 44 & $\begin{array}{l}\text { Artemisia capillaris } \\
\text { Thunb. }\end{array}$ & Yinchenhao茵陈蒿 & $m a^{6} 7 a i^{3}$ & Asteraceae & Herb & Wild & Whole plant & $\begin{array}{l}\text { Grinding, decoction; Taken orally for hepatitis } \\
\text { and jaundice }\end{array}$ \\
\hline 45 & $\begin{array}{l}\text { Artemisia japonica } \\
\text { Thunb. }\end{array}$ & Muhao牡蒿 & - & Asteraceae & Herb & Wild & Whole plant & $\begin{array}{l}\text { Grinding, decoction; Taken orally for clearing } \\
\text { away heat and toxic materials, inflammation } \\
\text { and blood stasis }\end{array}$ \\
\hline 46 & $\begin{array}{l}\text { Asarum longerhizomatosum } \\
\text { C. F. Liang et C. S. } \\
\text { Yang }\end{array}$ & $\begin{array}{l}\text { Xijingjin'erhuan长茎金 } \\
\text { 耳环 }\end{array}$ & - & Aristolochiaceae & Herb & Wild & Whole plant & $\begin{array}{l}\text { Grinding, decoction; Taken orally for headache, } \\
\text { toothache, cough, diarrhea, acute enteritis }\end{array}$ \\
\hline 47 & Asarum sieboldii Miq. & Xixin细辛 & ruon $^{2}$ nden ${ }^{5}$ hha $^{3}$ & Aristolochiaceae & Herb & Wild & Whole plant, Root & $\begin{array}{l}\text { Grinding, decoction; Taken orally for cough, } \\
\text { relieving pain }\end{array}$ \\
\hline 48 & $\begin{array}{l}\text { Asparagus } \\
\text { cochinchinensis } \\
\text { (Lour.) Merr. }\end{array}$ & Tianmendong天门冬 & $\operatorname{lak}^{5} \mathrm{~m}^{2} n^{2} \operatorname{tun}^{1}$ & Asparagaceae & Herb & Wild & Tuber & $\begin{array}{l}\text { Grinding, decoction; Taken orally for tuberculosis, } \\
\text { cough, constipation, diabetes and sore throat } \\
\text { after rash }\end{array}$ \\
\hline 49 & $\begin{array}{l}\text { Azolla imbricata } \\
\text { (Roxb.) Nakai }\end{array}$ & Manjianghong满江红 & - & Azollaceae & Herb & Wild & Whole plant & $\begin{array}{l}\text { Pound fresh part applied on the affected area, } \\
\text { treating for measles }\end{array}$ \\
\hline 50 & $\begin{array}{l}\text { Baphicacanthus } \\
\text { cusia (Nees) Bremek. }\end{array}$ & Banlan板蓝 & ruon ${ }^{2}$ wom 1 & Acanthaceae & Herb & Homegarden & Whole plant & $\begin{array}{l}\text { Grinding, decoction drunk for common cold, } \\
\text { sore throat, parotitis and epidemic cerebrospinal } \\
\text { meningitis }\end{array}$ \\
\hline
\end{tabular}


Table 1 Inventory of Medicinal Plants Traditionally Used by Maonan People (Continued)

\begin{tabular}{|c|c|c|c|c|c|c|c|c|}
\hline 51 & Bauhinia brachycarpa Wall. & $\begin{array}{l}\text { Anyeyangtijia鞍叶羊蹄 } \\
\text { 甲 }\end{array}$ & yanm ${ }^{3} g^{3} m^{3} d u 0^{5}$ & Fabaceae & Tree & Both & Root, Leaf & $\begin{array}{l}\text { Boiled with meat and drunk the soup, treating } \\
\text { for cough, hemoptysis }\end{array}$ \\
\hline 52 & $\begin{array}{l}\text { Bauhinia championii (Benth.) } \\
\text { Benth. }\end{array}$ & Longxuteng龙须藤 & bjeu $^{3} \mathrm{in}^{5}$ & Fabaceae & Liana & Both & Stem & $\begin{array}{l}\text { Grinding, decoction drunk for rheumatism, } \\
\text { traumatic injury, stomachache, waist and } \\
\text { legs pain }\end{array}$ \\
\hline 53 & $\begin{array}{l}\text { Belamcanda chinensis } \\
\text { (L.) Redoute }\end{array}$ & Shegan射干 & $m e i^{5} \operatorname{van}^{3} \mathrm{bi}^{2} \mathrm{n}^{2}$ & Iridaceae & Herb & Both & Root & Grinding, decoction drunk for sore throat \\
\hline 54 & $\begin{array}{l}\text { Berberis julianae } \\
\text { Schneid }\end{array}$ & Haozhuci豪猪刺 & ta: $m^{2} m \varepsilon n^{5} \operatorname{sem}^{5}$ & Berberidaceae & Shrub & Wild & Root & $\begin{array}{l}\text { Grinding, boiled with water and washed the } \\
\text { affected area for clearing away heat and } \\
\text { toxic materials, inflammation }\end{array}$ \\
\hline 55 & Bidens pilosa $\mathrm{L}$. & Guizhencao鬼针草 & wok $^{7}$ cut $^{7} n a^{5}$ & Asteraceae & Herb & Wild & Whole plant & $\begin{array}{l}\text { Grinding, decoction; Taken orally for nephritis, } \\
\text { jaundice, rheumatism, ostealgia, diarrhea, throat } \\
\text { ache, kidney deficiency and waist pain }\end{array}$ \\
\hline 56 & Bischofia javanica Bl. & Qiufeng秋枫 & - & Euphorbiaceae & Tree & Both & Stem, Leaf & $\begin{array}{l}\text { Grinding, decoction; Taken orallyfor removing } \\
\text { blood stasis, carminative, improving indigestion }\end{array}$ \\
\hline 57 & $\begin{array}{l}\text { Bletilla striata (Thunb. } \\
\text { ex A. Murray) Rchb. f. }\end{array}$ & Baiji白及 & kon'nat ${ }^{7}$ & Orchidaceae & Herb & Both & Bulb & $\begin{array}{l}\text { Powder swallowed for tuberculosis and } \\
\text { empyrosis }\end{array}$ \\
\hline 58 & $\begin{array}{l}\text { Blumea balsamifera } \\
\text { (L.) DC. }\end{array}$ & Ainaxiang艾纳香 & ruon ${ }^{2} n n a i^{6} l a u^{4}$ & Asteraceae & Herb & Wild & Whole plant & $\begin{array}{l}\text { Grinding, decoction; Taken orally for common } \\
\text { cold, rheumatic arthritis, traumatic injury, } \\
\text { dysmenorrhea and afterpains }\end{array}$ \\
\hline 59 & $\begin{array}{l}\text { Boehmeria nivea } \\
\text { (L.) Gaudich. }\end{array}$ & Zhuma草麻 & $m b a^{3} n a n^{3}$ & Urticaceae & Shrub & Wild & Root Bark, Leaf & $\begin{array}{l}\text { Grinding, decoction; Taken orally for miscarriage } \\
\text { prevention, hematuria, traumatic injury, bone } \\
\text { fracture, diuresis, measles, joint sprain }\end{array}$ \\
\hline 60 & Bombax malabaricum DC. & Mumian木棉 & wai ${ }^{5} e^{4}$ & Bombacaceae & Tree & Both & $\begin{array}{l}\text { Flower, Root bark, } \\
\text { Root }\end{array}$ & $\begin{array}{l}\text { Grinding, decoction; Taken orally; Flower is } \\
\text { treating for enteritis, stomach ulcer; Root } \\
\text { bark is treating for rheumatism, traumatic } \\
\text { injury; Root is treating for chronic nephritis } \\
\text { gastricism, stomach ulcer, tuberculosis of } \\
\text { cervical lymph nodes }\end{array}$ \\
\hline 61 & $\begin{array}{l}\text { Botrychium ternatum } \\
\text { (Thunb.) Sw. }\end{array}$ & Yindijue阴地蕨 & $\mathrm{do}^{5} \mathrm{~g} 7 \mathrm{~m}^{2} \mathrm{da}: \mathrm{n}^{3}$ & Botrychiaceae & Herb & Wild & Whole plant & Grinding, decoction; Taken orally for cough \\
\hline 62 & $\begin{array}{l}\text { Brucea javanica } \\
\text { (L.) Merr. }\end{array}$ & Yadanzi鸦胆子 & - & Simaroubaceae & Shrub & Wild & Seed & $\begin{array}{l}\text { Grinding, decoction; Taken orally for diarrhea, } \\
\text { malaria and chromic diarrhea }\end{array}$ \\
\hline 63 & $\begin{array}{l}\text { Bryophyllum pinnatum } \\
\text { (L. f.) Oken }\end{array}$ & $\begin{array}{l}\text { Luoyeshenggen落地生 } \\
\text { 根 }\end{array}$ & $r_{u o n}^{2} \mathrm{ra}^{2} \mathrm{pu}^{3}$ & Crassulaceae & Herb & Wild & Whole plant & $\begin{array}{l}\text { Pound fresh part applied on the affected area, } \\
\text { treating for detumescence by detoxification, } \\
\text { promoting blood circulation to arrest pain, } \\
\text { draw out pus and toxin }\end{array}$ \\
\hline 64 & $\begin{array}{l}\text { Buddleja officinalis } \\
\text { Maxim. }\end{array}$ & Mimenghua密蒙花 & $w a^{3} k_{n}{ }^{3} \mathrm{ruo}^{2}$ & Loganiaceae & Shrub & Wild & Flower & $\begin{array}{l}\text { Grinding, decoction; Taken orally for swelling } \\
\text { and pain of eye, hyperdacryosis and cloudness } \\
\text { of cornea }\end{array}$ \\
\hline 65 & $\begin{array}{l}\text { Caesalpinia sappan } \\
\text { Linn. }\end{array}$ & Sumu苏木 & $m e^{4} \mathrm{sam}^{3} \mathrm{mok}^{8}$ & Fabaceae & Tree & Wild & Heartwood & $\begin{array}{l}\text { Grinding, decoction; Taken orally for traumatic } \\
\text { injury, rheumatism, ostealgia, bleeding }\end{array}$ \\
\hline
\end{tabular}


Table 1 Inventory of Medicinal Plants Traditionally Used by Maonan People (Continued)

\begin{tabular}{|c|c|c|c|c|c|c|c|c|}
\hline 66 & $\begin{array}{l}\text { Caesalpinia sepiaria } \\
\text { Roxb. }\end{array}$ & Yunshi云实 & ?ทәn ?niao $^{2}$ & Fabaceae & Tree & Wild & Root, Seed & $\begin{array}{l}\text { Medicinal liquor for treating contraception in } \\
\text { the menstrual period }\end{array}$ \\
\hline 67 & Callicarpa macrophylla Vahl & Dayezizhu大叶紫珠 & ruon$^{2} l a k^{8}$ phau $^{5}$ & Verbenaceae & Shrub & Wild & Root, Leaf & $\begin{array}{l}\text { Grinding, decoction; Taken orally for hemafecia } \\
\text { and hemoptysis }\end{array}$ \\
\hline 68 & Campanumoea javanica Bl. & Jianqianbao金钱豹 & bieuthw $\omega p^{8}$ jou ${ }^{1}$ & Campanulaceae & Herb & Wild & Root & $\begin{array}{l}\text { Powdered and swallowed for tuberculosis, } \\
\text { enteritis, diarrhea, appendicitis, traumatic } \\
\text { injury and piles }\end{array}$ \\
\hline 69 & $\begin{array}{l}\text { Camptotheca acuminata } \\
\text { Decne. }\end{array}$ & Xishu喜树 & - & Nyssaceae & Tree & Both & Fruit, Root & $\begin{array}{l}\text { Grinding, decoction; Taken orally for cancer } \\
\text { and schistosome }\end{array}$ \\
\hline 70 & $\begin{array}{l}\text { Canscora lucidissima } \\
\text { (Levl. et Vaniot) Hand.-Mazz }\end{array}$ & Chuanxincao穿心草 & $m a^{6}$ chuan $^{2}$ & Gentianaceae & Herb & Wild & Whole plant & $\begin{array}{l}\text { Grinding, decoction; Taken orally for stranguria, } \\
\text { snake bite, stomachache, cough and jaundiced } \\
\text { hepatitis }\end{array}$ \\
\hline 71 & $\begin{array}{l}\text { Capsella bursa-pastoris } \\
\text { (L.) Medik. }\end{array}$ & Ji荠 & $m b a^{3} k ⿰ \eta^{1} p^{3}{ }^{3}$ & Cruciferae & Herb & Wild & Whole plant & $\begin{array}{l}\text { Grinding, decoction; Taken orally for catching } \\
\text { common cold, fever, nephritis, edema, } \\
\text { hypertension, enteritis }\end{array}$ \\
\hline 72 & Cassia tora Linn. & Jueming决明 & thou ${ }^{6} \operatorname{man}^{3} \times 13 \eta^{3}$ & Fabaceae & Herb & Both & Seed & $\begin{array}{l}\text { Grinding, decoction; Taken orally for } \\
\text { hyperlipidemia, hepatitis, stomachache, } \\
\text { acute conjunctivitis, habitual constipation, } \\
\text { dental ulcer }\end{array}$ \\
\hline 73 & Cassytha filiformis $\mathrm{L}$. & Wugenteng无根藤 & bieu $^{3}$ chim $^{6}$ cieu $^{1}$ & Lauraceae & Herb & Wild & Stem & $\begin{array}{l}\text { Grinding, decoction; Taken orally for vitiligo, } \\
\text { jaundice, constipation, waist and knees pain, } \\
\text { impotence and spermatorrhea }\end{array}$ \\
\hline 74 & $\begin{array}{l}\text { Cayratia japonica (Thunb.) } \\
\text { Gagnep. }\end{array}$ & Wulianmei乌誉莓 & - & Vitaceae & Herb & Wild & Whole plant, Root & Medicinal liquor for paralysis \\
\hline 75 & Celosia argentea $\mathrm{L}$. & Qingxiang青葙 & $m b a^{3} p w m^{3} p a^{5}$ & Amaranthaceae & Herb & Wild & Whole plant & $\begin{array}{l}\text { Grinding, decoction; Taken orally for trachitis, } \\
\text { gastricism }\end{array}$ \\
\hline 76 & $\begin{array}{l}\text { Cerastium glomeratum } \\
\text { Thuill. }\end{array}$ & Qiuxujuan'er球序卷耳 & ma: ${ }^{6} \operatorname{Tan}^{4} z_{a u^{2}}$ & Caryophyllaceae & Herb & Wild & Whole plant & $\begin{array}{l}\text { Pound fresh part applied on the affected area, } \\
\text { treating for febrile convulsion }\end{array}$ \\
\hline 77 & $\begin{array}{l}\text { Chaenomeles sinensis } \\
\text { (Thouin) Koehne }\end{array}$ & Mugua木瓜 & - & Rosaceae & Shrub & Homegarden & Fruit & $\begin{array}{l}\text { Grinding, decoction; Taken orally for smooth } \\
\text { the liver and stomach }\end{array}$ \\
\hline 78 & Chirita eburnea Hance & Niu'erduo牛耳朵 & $m a^{5} b a^{5}$ & Gesneriaceae & Herb & Wild & Whole plant & Grinding, decoction; Taken orally for bronchitis \\
\hline 79 & $\begin{array}{l}\text { Chloranthus holostegius } \\
\text { (Handel-Mazzetti) Pei \& Shan }\end{array}$ & $\begin{array}{l}\text { Quanyuanjinlilan全缘金 } \\
\text { 栗兰 }\end{array}$ & tei $^{3} k_{u a i}{ }^{5} \mathrm{wa}^{5}$ & Chloranthaceae & Herb & Wild & Root & $\begin{array}{l}\text { Boiled with meat and drunk the soup, treating } \\
\text { for weakness }\end{array}$ \\
\hline 80 & $\begin{array}{l}\text { Cinnamomum camphora } \\
\text { (L.) Presl }\end{array}$ & Xiangzhang香樟 & $\mathrm{mei}^{4} \mathrm{kau}^{1}$ & Lauraceae & Tree & Homegarden & Bark & $\begin{array}{l}\text { Grinding, decoction; Taken orally for acute } \\
\text { gastroenteritis, rheumatism, ostealgia, emesis, } \\
\text { diarrhea and bone fracture }\end{array}$ \\
\hline 81 & Cinnamomum cassia Presl & Rougui肉桂 & - & Lauraceae & Tree & Homegarden & Stem & $\begin{array}{l}\text { Grinding, decoction; Taken orally for cough, } \\
\text { dysmenorrhea and sweating }\end{array}$ \\
\hline 82 & Cinnamomum subavenium & Xianggui香桂 & - & Lauraceae & Tree & Both & Bark & Grinding, decoction; Taken orally for antiseptic \\
\hline
\end{tabular}


Table 1 Inventory of Medicinal Plants Traditionally Used by Maonan People (Continued)

\begin{tabular}{|c|c|c|c|c|c|c|c|c|}
\hline 83 & $\begin{array}{l}\text { Cirsium japonicum Fisch. } \\
\text { ex DC. }\end{array}$ & Daji大蓟 & $\mathrm{mba}^{3} \operatorname{tin}^{3} \mathrm{tsuok}^{7} \operatorname{lau}^{4}$ & Asteraceae & Herb & Wild & Root, Whole plant & $\begin{array}{l}\text { Grinding, decoction; Taken orally for jaundice, } \\
\text { scabies, hemafecia, muscle swelling and } \\
\text { gastroduodenal ulcer }\end{array}$ \\
\hline 84 & Clematis chinensis Osbeck. & Weilingxian威灵仙 & ruon $^{2}$ pek $^{7} \mathrm{mi}^{6} \operatorname{san}^{3}$ & Ranunculaceae & Liana & Wild & Root, Leaf & $\begin{array}{l}\text { Grinding, decoction; Taken orally for tonsillitis, } \\
\text { jaundice, migraine and rheumatism }\end{array}$ \\
\hline 85 & $\begin{array}{l}\text { Clerodendrum chinense } \\
\text { (Osbeck) Mabb. }\end{array}$ & Choumoli臭茉莉 & ruon $^{2}$ phun $^{6} \mathrm{hi}^{6}$ & Verbenaceae & Shrub & Wild & Whole plant & $\begin{array}{l}\text { Pound fresh part applied on the affected area, } \\
\text { treating for rheumatic arthritis, traumatic injury, } \\
\text { rheumatism and detumescence }\end{array}$ \\
\hline 86 & $\begin{array}{l}\text { Clerodendrum cyrtophyllum } \\
\text { Turcz. }\end{array}$ & Daqing大青 & - & Verbenaceae & Shrub & Wild & Leaf & $\begin{array}{l}\text { Ground, decoction; Taken orally for fever due } \\
\text { to common cold, tonsillitis, pharyngitis, parotitis, } \\
\text { enteritis and diarrhea }\end{array}$ \\
\hline 87 & Coix lacryma-jobi L. & Yiyi薏蔌 & yhou gan $^{5} y \partial^{4}$ & Gramineae & Herb & Both & Root & $\begin{array}{l}\text { Grinding, decoction; Taken orally for acute } \\
\text { nephritis }\end{array}$ \\
\hline 88 & Colocasia antiquorum Schott & Yeyu野芋 & phi $^{2} n i \partial \eta^{6}$ & Araceae & Herb & Wild & Tuber & $\begin{array}{l}\text { Pound fresh part applied on the affected area, } \\
\text { treating for bleeding, furuncle, empyrosis and } \\
\text { snake bite }\end{array}$ \\
\hline 89 & $\begin{array}{l}\text { Commelina } \\
\text { communis L. }\end{array}$ & Yazhicao鸭趾草 & $\mathrm{mba}^{3} \mathrm{ciap}^{7}$ & Commelinaceae & Herb & Wild & Whole plant & $\begin{array}{l}\text { Grinding, decoction; Taken orally for diarrhea, } \\
\text { influenza, acute tonsillitis, edema, enteritis, } \\
\text { urinary tract infection, empyrosis and bleeding }\end{array}$ \\
\hline 90 & Crataegus pinnatifidaBunge & Shanzha山楂 & $\mathrm{d} \varepsilon \eta^{1} m i \varepsilon^{52} y \partial^{2}$ & Rosaceae & Tree & Homegarden & Fruit & $\begin{array}{l}\text { Grinding, decoction; Taken orally for poor } \\
\text { appetite, blood stasis }\end{array}$ \\
\hline 91 & Croton tiglium L. & Badou巴豆 & ruon $^{2} \mathrm{mei}^{4} \mathrm{miət}^{7}$ & Euphorbiaceae & Tree & Homegarden & Root Bark, Leaf & $\begin{array}{l}\text { Pound fresh leaf applied on the affected } \\
\text { area, treating for bleeding, herpes zoster; } \\
\text { Pound root bark applied on the affected } \\
\text { area, treating for snake bite }\end{array}$ \\
\hline 92 & Cucumis sativus $\mathrm{L}$. & Huanggua黄瓜 & - & Cucurbitaceae & Herb & Homegarden & Fruit & $\begin{array}{l}\text { Pound fresh part applied on the affected } \\
\text { area, treating for bleeding and skin whitening }\end{array}$ \\
\hline 93 & Curculigo orchioides Gaertn. & Xianmao仙茅 & ruon $^{2} \operatorname{san}^{3}$ thok $^{8}$ & Amaryllidaceae & Herb & Homegarden & Whole plant & $\begin{array}{l}\text { Grinding, decoction; Taken orally for headache } \\
\text { due to common cold, rheumatic arthritis, } \\
\text { neurasthenia, chronic nephritis, erectile } \\
\text { dysfunction and seminal leakage }\end{array}$ \\
\hline 94 & Curcuma aromatica Salisb. & Yujin郁金 & - & Zingiberaceae & Herb & Wild & Tuber & $\begin{array}{l}\text { Grinding, decoction; Taken orally for bleeding, } \\
\text { jaundice and cooling blood }\end{array}$ \\
\hline 95 & Curcuma longa $\mathrm{L}$. & Jianghuang姜黄 & ruon $^{2}\left(\varepsilon \eta^{3}\right.$ won $^{2}$ & Zingiberaceae & Herb & Homegarden & Rhizome & $\begin{array}{l}\text { Grinding, decoction; Taken orally for abnormal } \\
\text { menstruation, amenorrhea, flatulence and } \\
\text { blood stasis }\end{array}$ \\
\hline 96 & $\begin{array}{l}\text { Curcuma zedoaria (Christm.) } \\
\text { Roscoe }\end{array}$ & E'zhu莪术 & $\mathrm{pi}^{6} \mathrm{c \varepsilon n}^{3} \mathrm{n} \mathrm{m}^{3}$ & Zingiberaceae & Herb & Homegarden & Rhizome & $\begin{array}{l}\text { Grinding, decoction; Taken orally for rheumatism, } \\
\text { ostealgia, traumatic injury, abdomen pain }\end{array}$ \\
\hline 97 & Cuscuta chinensis & Tusizi莬丝子 & - & Convolvulaceae & Herb & Wild & Whole plant & Grinding, decoction; Taken orally for hepatitis \\
\hline
\end{tabular}


Table 1 Inventory of Medicinal Plants Traditionally Used by Maonan People (Continued)

\begin{tabular}{|c|c|c|c|c|c|c|c|c|}
\hline 98 & $\begin{array}{l}\text { Cyclea hypoglauca } \\
\text { (Schauer) } \\
\text { Diels }\end{array}$ & $\begin{array}{l}\text { Fenyelunhuanteng粉叶 } \\
\text { 轮环藤 }\end{array}$ & - & Menispermaceae & Liana & Wild & Root & $\begin{array}{l}\text { Grinding, decoction; Taken orally for toothache, } \\
\text { urinary tract infection, rheumatism, diphtheria, } \\
\text { ostealgia; Pound fresh part applied on the } \\
\text { affected area, treating for carbuncle, snake bite }\end{array}$ \\
\hline 99 & Cynanchum atratum Bunge & Baiwei白薇 & $\left.\operatorname{lau}^{2} \operatorname{tgin}^{5} x\right|^{5}$ & Asclepiadaceae & Herb & Wild & Root & Medicinal liquor for treating rheumatic arthritis \\
\hline 100 & $\begin{array}{l}\text { Cynanchum auriculatum Royle } \\
\text { ex Wight }\end{array}$ & Niupixiao牛皮消 & $g ? \varepsilon^{2} \operatorname{lin}^{5} \times i a o^{5}$ & Asclepiadaceae & Shrub & Wild & Root & $\begin{array}{l}\text { Boiled with meat and drunk the soup, treating } \\
\text { for infantile dry-sickness and malnutrition }\end{array}$ \\
\hline 101 & $\begin{array}{l}\text { Cynanchum officinale (Hemsl.) } \\
\text { Tsiang \& H.D.Zhang }\end{array}$ & Zhushateng朱砂藤 & - & Asclepiadaceae & Shrub & Wild & Root & $\begin{array}{l}\text { Grinding, decoction; Taken orally for pain killer } \\
\text { and weakness }\end{array}$ \\
\hline 102 & $\begin{array}{l}\text { Cynanchum paniculatum } \\
\text { (Bunge) Kitag. }\end{array}$ & Xuchangqing徐长卿 & $\operatorname{ta}^{6} ? n u^{2}$ & Asclepiadaceae & Herb & Both & Root, Whole plant & $\begin{array}{l}\text { Grinding, decoction; Taken orally for enteritis } \\
\text { and diarrhea }\end{array}$ \\
\hline 103 & Cyperus rotundus $\mathrm{L}$. & Xiangfuzi香附子 & $\operatorname{lak}^{8} \mathrm{rut}^{8}$ & Cyperaceae & Herb & Wild & Tuber & $\begin{array}{l}\text { Grinding, decoction; Taken orally for clearing } \\
\text { and activating the channels and collaterals, } \\
\text { common cold, abnormal menstruation }\end{array}$ \\
\hline 104 & $\begin{array}{l}\text { Cyrtomium fortunei } \\
\text { J. Sm. }\end{array}$ & Guanzhong贯众 & $\operatorname{rin}^{3} \operatorname{tsi\varepsilon k}^{7} \operatorname{lau}^{4}$ & Dryopteridaceae & - & Wild & Whole plant & $\begin{array}{l}\text { Grinding, decoction; Taken orally for common } \\
\text { cold, parotitis, gastrorrhagia, hematuria, } \\
\text { postpartum lochiorrhea and body deficiency } \\
\text { disease }\end{array}$ \\
\hline 105 & Datura metel L. & Yangjinhua洋金花 & - & Solanaceae & Herb & Wild & Flower & $\begin{array}{l}\text { Pound and applied on the affected area for } \\
\text { ulcer and pains }\end{array}$ \\
\hline 106 & Datura stramonium $\mathrm{L}$. & Mantuoluo曼陀罗 & ruon $^{2}$ chou $^{6} \mathrm{dun}^{3}$ & Solanaceae & Herb & Wild & Leaf & $\begin{array}{l}\text { Pound fresh part applied on the affected area, } \\
\text { treating for furuncle and traumatic injury }\end{array}$ \\
\hline 107 & $\begin{array}{l}\text { Davallia mariesii } \\
\text { T. Moore ex Baker }\end{array}$ & Gusuibu骨碎补 & $x \operatorname{in}^{5} b o a^{5}$ & Davalliaceae & - & Wild & Rhizome & $\begin{array}{l}\text { Grinding, decoction; Taken orally for bone } \\
\text { fracture and fructus psoraleae }\end{array}$ \\
\hline 108 & $\begin{array}{l}\text { Desmodium } \\
\text { heterocarpon (L.) DC. }\end{array}$ & Jiadidou假地豆 & thou $\mathrm{ti}^{5} \mathrm{pa}^{5}$ & Fabaceae & Shrub & Wild & Whole plant & $\begin{array}{l}\text { Grinding, decoction; Taken orally for preventing } \\
\text { mumps, epidemic encephalitis B, kidney and } \\
\text { vesical stone }\end{array}$ \\
\hline 109 & $\begin{array}{l}\text { Dichondra repens J.R. Forst. } \\
\& \text { G. Forst. }\end{array}$ & Matijin马蹄金 & ruon$^{2} \operatorname{tin}^{3} \mathrm{mia}^{4}$ & Convolvulaceae & Herb & Wild & Whole plant & $\begin{array}{l}\text { Pound fresh part applied on the affected area, } \\
\text { treating for bleeding, urinary stone and } \\
\text { jaundiced hepatitis }\end{array}$ \\
\hline 110 & $\begin{array}{l}\text { Dicliptera chinensis } \\
\text { (L.) Juss. }\end{array}$ & Gougancai狗肝菜 & ruon $\operatorname{təp}^{7} \mathrm{ma}^{3}$ & Acanthaceae & Herb & Wild & Whole plant & $\begin{array}{l}\text { Grinding, decoction; Taken orally for fever due } \\
\text { to common cold, epidemic hepatitis B, rheumatic } \\
\text { arthritis, conjunctivitis, diuresis and measles }\end{array}$ \\
\hline 111 & $\begin{array}{l}\text { Dimocarpus longan } \\
\text { Lour. }\end{array}$ & Longyan龙眼 & ruon $^{2}$ kuei $^{4}$ juon $^{2}$ & Sapindaceae & Tree & Homegarden & Aril & $\begin{array}{l}\text { Medicinal liquor for cosmetic, insomnia, } \\
\text { forgetfulness, replenishing heart, tonic } \\
\text { and blood deficiency }\end{array}$ \\
\hline 112 & Dioscorea bulbifera L. & Huangdu黄独 & lak $^{8}$ phuo $^{2}$ & Dioscoreaceae & Liana & Homegarden & Tuber & $\begin{array}{l}\text { Grinding, decoction; Taken orally for cough, } \\
\text { hemoptysis and epistaxis }\end{array}$ \\
\hline 113 & $\begin{array}{l}\text { Dioscorea cirrhosa } \\
\text { Lour. }\end{array}$ & Shuliang薯莨 & dan ${ }^{5} g 7 u i^{5} p \varepsilon^{2}$ & Dioscoreaceae & Liana & Homegarden & Tuber & $\begin{array}{l}\text { Grinding, decoction; Taken orally for } \\
\text { gastroduodenal ulcer }\end{array}$ \\
\hline 114 & Dioscorea opposita Thunb. & Shuyu薯蓣 & - & Dioscoreaceae & Liana & Homegarden & Tuber & $\begin{array}{l}\text { Grinding, decoction; Taken orally for weakness, } \\
\text { cough and frequent urination }\end{array}$ \\
\hline
\end{tabular}


Table 1 Inventory of Medicinal Plants Traditionally Used by Maonan People (Continued)

\begin{tabular}{|c|c|c|c|c|c|c|c|c|}
\hline 115 & Diospyros kaki Thunb. & Shi柿 & $\operatorname{den}^{3} \operatorname{mian}^{5}$ & Ebenaceae & Tree & Homegarden & $\begin{array}{l}\text { Fruit, Persistent } \\
\text { calyx }\end{array}$ & $\begin{array}{l}\text { Pound fresh part mixing the rice wine applied on } \\
\text { the affected area, treating for lymphadenectasis }\end{array}$ \\
\hline 116 & $\begin{array}{l}\text { Dipsacus asperoides C.Y. } \\
\text { Cheng \& Ai }\end{array}$ & Chuanxuduan川续断 & $n_{n} n^{2} b u^{2} y \varepsilon^{5}$ & Dipsacaceae & Herb & Wild & Seed, Root & $\begin{array}{l}\text { Boiled with meat and drunk the soup, treating } \\
\text { for leucorrhoea and bone fracture }\end{array}$ \\
\hline 117 & $\begin{array}{l}\text { Disporum cantoniense } \\
\text { (Lour.) Merr. }\end{array}$ & Wanshouzhu万寿竹 & $m a^{6} m e^{5} v \varepsilon n^{3}$ & Liliaceae & Herb & Wild & Root, Rhizome & $\begin{array}{l}\text { Boiled with meat and drunk the soup, treating } \\
\text { for cough }\end{array}$ \\
\hline 118 & $\begin{array}{l}\text { Drynaria propinqua } \\
\text { (Wall. ex Mett.) J. Sm. }\end{array}$ & $\begin{array}{l}\text { Shilianjianghujue石莲姜 } \\
\text { 葪蕨 }\end{array}$ & - & Drynariaceae & - & Wild & Rhizome & $\begin{array}{l}\text { Medicinal liquor for treating rheumatic arthritis, } \\
\text { traumatic injury, bone fracture and blood stasis }\end{array}$ \\
\hline 119 & $\begin{array}{l}\text { Duchesnea indica (Andrews) } \\
\text { Teschem. }\end{array}$ & Shemei蛇莓 & ta:nm²bei²zen' & Rosaceae & Herb & Wild & Whole plant & $\begin{array}{l}\text { Pound fresh part applied on the affected area, } \\
\text { treating for empyrosis, snake bite, furuncle }\end{array}$ \\
\hline 120 & $\begin{array}{l}\text { Dysosma versipellis (Hance) } \\
\text { M. Cheng ex T.S. Ying }\end{array}$ & Bajiaolian八角莲 & va ${ }^{5}$ piat $^{7} \lim ^{6}$ & Berberidaceae & Herb & Both & Rhizome & $\begin{array}{l}\text { Grinding, decoction; Taken orally for mumps, } \\
\text { traumatic injury, lymphnoditis, snake bite, } \\
\text { breast carcinoma }\end{array}$ \\
\hline 121 & $\begin{array}{l}\text { Eclipta prostrata } \\
\text { (L.) L. }\end{array}$ & Lichang鳢肠 & $w o k^{7} m ə k^{8}$ & Asteraceae & Herb & Wild & Whole plant & $\begin{array}{l}\text { Grinding, decoction; Taken orally for infantile } \\
\text { diarrhea, enteritis, hemafecia, hematuria, } \\
\text { hemoptysis and bleeding }\end{array}$ \\
\hline 122 & Elephantopus scaber $\mathrm{L}$. & Didancao地胆草 & ruon ${ }^{2} \operatorname{tap}^{7} \mathrm{do}^{6}$ & Asteraceae & Herb & Wild & Whole plant & $\begin{array}{l}\text { Grinding, decoction; Taken orally for common } \\
\text { cold, acute tonsillitis, acute jaundiced hepatitis, } \\
\text { ascites due to cirrhosis, chronic gastricism and } \\
\text { furuncle }\end{array}$ \\
\hline 123 & $\begin{array}{l}\text { Eleusine indica (L.) } \\
\text { Gaertn. }\end{array}$ & Niujincao牛筋草 & ruon $^{2} \mathrm{su}^{5} \mathrm{chin}^{6}$ & Gramineae & Herb & Wild & Whole plant & $\begin{array}{l}\text { Grinding, decoction; Taken orally for traumatic } \\
\text { injury, rheumatism, ostealgia, infantile indigestion }\end{array}$ \\
\hline 124 & $\begin{array}{l}\text { Emilia sonchifolia } \\
\text { (L.) DC. }\end{array}$ & Yidianhong一点红 & $m b a^{3} k h a^{3} t^{5}$ & Asteraceae & Herb & Wild & Whole plant & $\begin{array}{l}\text { Grinding, decoction; Taken orally for } \\
\text { inflammation, sore throat, cough, fever } \\
\text { due to common cold, urticaria, herpes zoster }\end{array}$ \\
\hline 125 & $\begin{array}{l}\text { Epimedium brevicornu } \\
\text { Maximowicz Trudy } \\
\text { Imp. S.-Peterburgsk. }\end{array}$ & Yinyanghuo淫羊藿 & $m a^{5} g^{2} n^{2} u o^{3}$ & Berberidaceae & Herb & Both & Stem, Leaf & Medicinal liquor for treating rheumatism, tonic \\
\hline 126 & Epimeredi indica (L.) Rothm. & Guangfangfen广防风 & ruon$^{2} w o \eta^{2}$ & Lamiaceae & Herb & Wild & Whole plant & $\begin{array}{l}\text { Grinding, decoction; Taken orally for common } \\
\text { cold, acute gastroenteritis; boiled with water } \\
\text { and washed the affected area for snake bite, } \\
\text { furuncle, eczema }\end{array}$ \\
\hline 127 & Equisetum arvense $\mathrm{L}$. & Wenjing问荆 & $g O \eta^{2} d a u^{5}$ & Equisetaceae & - & Wild & Whole plant & Powder swallowed for headache \\
\hline 128 & Equisetum hyemale L. & Bitongcao笔筒草 & - & Equisetaceae & - & Wild & Whole plant & $\begin{array}{l}\text { Grinding, decoction; Taken orally for bleeding, } \\
\text { diuresis }\end{array}$ \\
\hline 129 & $\begin{array}{l}\text { Eriobotrya japonica (Thunb.) } \\
\text { Lindl. }\end{array}$ & Pipa枇杷 & $v a^{3} \mathrm{bi}^{2} \mathrm{ba} \mathrm{a}^{5}$ & Rosaceae & Tree & Homegarden & Leaf & $\begin{array}{l}\text { Grinding, decoction; Taken orally for pertussis } \\
\text { cough }\end{array}$ \\
\hline 130 & Eucalyptus robusta $\mathrm{Sm}$. & An桉 & $m e i^{4} \mathrm{Cau}^{5} \times u i^{4}$ & Myrtaceae & Tree & Homegarden & Leaf & $\begin{array}{l}\text { Grinding, decoction; Taken orally for influenza, } \\
\text { diarrhea }\end{array}$ \\
\hline 131 & Eucommia ulmoides Oliv. & Duzhong杜仲 & thu $^{6} \operatorname{tsun}^{5}$ & Eucommiaceae & Tree & Both & Bark & $\begin{array}{l}\text { Grinding, decoction; Taken orally for hypertension, } \\
\text { kidney deficiency, lumbago }\end{array}$ \\
\hline
\end{tabular}


Table 1 Inventory of Medicinal Plants Traditionally Used by Maonan People (Continued)

\begin{tabular}{|c|c|c|c|c|c|c|c|c|}
\hline 132 & Eupatorium chinense $\mathrm{L}$. & Duoxugong多须公 & - & Asteraceae & Herb & Wild & Root & $\begin{array}{l}\text { Grinding, decoction; Taken orally for clearing } \\
\text { away heat and toxic materials, blood stasis, } \\
\text { traumatic injury }\end{array}$ \\
\hline 133 & Euphorbia antiquorum L. & Huoyangle火殊勒 & ruon $^{2} \mathrm{ko}^{3} \mid \mathrm{In}^{2}$ & Euphorbiaceae & Shrub & Wild & Whole plant & $\begin{array}{l}\text { Pound fresh part and fried with wine, applied on } \\
\text { the affected area, treating for furuncle, } \\
\text { innominate inflanunatory of unknown origin }\end{array}$ \\
\hline 134 & $\begin{array}{l}\text { Euphorbia chrysocoma } \\
\text { H. Lév. \& Vaniot }\end{array}$ & Shuihuanghua水黄花 & $m a:^{3} n o m^{2} 7 a n^{2}$ & Euphorbiaceae & Herb & Wild & Root & $\begin{array}{l}\text { Grinding, decoction; Taken orally for infectious } \\
\text { hepatitis }\end{array}$ \\
\hline 135 & Euphorbia hirta L. & Feiyangcao 飞扬草 & ruon $^{2} j \varepsilon n^{3}$ thun $^{6}$ thin $^{6}$ & Euphorbiaceae & Herb & Wild & Whole plant & $\begin{array}{l}\text { Grinding, decoction; Taken orally for bacillary } \\
\text { diarrhea, enteritis, bronchitis, nephritis }\end{array}$ \\
\hline 136 & $\begin{array}{l}\text { Euphorbia humifusa } \\
\text { Willdenow }\end{array}$ & Dijin地锦 & - & Euphorbiaceae & Herb & Wild & Whole plant & $\begin{array}{l}\text { Grinding, decoction; Taken orally for malaria, } \\
\text { diuresis }\end{array}$ \\
\hline 137 & $\begin{array}{l}\text { Euphorbia milii Des } \\
\text { Moul. }\end{array}$ & Tiehaitang铁海棠 & ruon $^{2} n d u n n^{3}$ wan $^{3}$ & Euphorbiaceae & Shrub & Both & Whole plant & $\begin{array}{l}\text { Pound fresh part applied on the affected area, } \\
\text { treating for carbuncle }\end{array}$ \\
\hline 138 & Euphorbia thymifolia L. & Qian'gencao千根草 & - & Euphorbiaceae & Herb & Wild & Whole plant & $\begin{array}{l}\text { Grinding, decoction; Taken orally for bacillary } \\
\text { diarrhea, enteritis, diarrhea, piles, bleeding }\end{array}$ \\
\hline 139 & $\begin{array}{l}\text { Evodia lepta (Spreng.) } \\
\text { Merr. }\end{array}$ & Sanyaku三桠苦 & ruon $^{2} \operatorname{sam}^{3}$ tsha $^{3}$ & Rutaceae & Tree & Wild & Root, Leaf & $\begin{array}{l}\text { Grinding, decoction; Taken orally for epidemic } \\
\text { meningitis, influenza, fever, epidemic } \\
\text { encephalitis B }\end{array}$ \\
\hline 140 & $\begin{array}{l}\text { Evodia rutaecarpa } \\
\text { (Juss.) Benth. }\end{array}$ & Wuzhuyu吴茱英 & tsha $a^{6} a^{6}$ & Rutaceae & Shrub & Wild & Fruit & $\begin{array}{l}\text { Grinding, decoction; Taken orally for diarrhea, } \\
\text { abnormal menstruation, diseases of liver stasis, } \\
\text { emesis }\end{array}$ \\
\hline 141 & $\begin{array}{l}\text { Fagopyrum tataricum } \\
\text { (L.) Gaertn. }\end{array}$ & Kuqiao苦养 & - & Polygonaceae & Herb & Wild & Fruit & $\begin{array}{l}\text { Grinding, decoction; Taken orally for } \\
\text { stomachache, indigestion }\end{array}$ \\
\hline 142 & $\begin{array}{l}\text { Fallopia multiflora } \\
\text { (Thunb.) Haraldson }\end{array}$ & Heshouwu何首乌 & $m ə n^{6} d a n^{3} y \varepsilon^{5}$ & Polygonaceae & Herb & Both & Tuber, Stem & Grinding, decoction; Taken orally for weakness \\
\hline 143 & Fibraurea recisa Pierre & Tianxianteng天仙藤 & - & Menispermaceae & Liana & Wild & Root & $\begin{array}{l}\text { Grinding, decoction; Taken orally for headache, } \\
\text { fever, acute tonsillitis, strep throat, diarrhea, } \\
\text { jaundiced hepatitis, gastricism, enteritis }\end{array}$ \\
\hline 144 & Ficus microcarpa L. f. & Rongshu榕树 & ruon $^{2} \mathrm{mei}^{4} \mathrm{jon}^{2}$ & Moraceae & Tree & Homegarden & Leaf & $\begin{array}{l}\text { Grinding, decoction; Taken orally for flu, malaria, } \\
\text { bronchitis, acute enteritis, bacillary diarrhea, } \\
\text { pertussis cough, tonsillitis }\end{array}$ \\
\hline 145 & Ficus tikoua Bureau & Diguo地果 & - & Moraceae & Liana & Wild & Whole plant & $\begin{array}{l}\text { Grinding, decoction; Taken orally for jaundice, } \\
\text { diarrhea and internal injury }\end{array}$ \\
\hline 146 & $\begin{array}{l}\text { Flemingia prostrata } \\
\text { Roxb. }\end{array}$ & Qianjinba千斤拔 & ruon $^{2} s \omega t^{7} k^{2} i^{2}$ & Fabaceae & Shrub & Wild & Root & $\begin{array}{l}\text { Grinding, decoction or medicinal liquor drunk } \\
\text { for lumbar muscle degeneration, traumatic } \\
\text { injury, rheumatic arthritis and tonsillitis }\end{array}$ \\
\hline 147 & $\begin{array}{l}\text { Gardenia jasminoides } \\
\text { J. Ellis }\end{array}$ & Zhizi梔子 & $l a k^{8} k \mathrm{ei}^{3}$ & Rubiaceae & Shrub & Both & Fruit & $\begin{array}{l}\text { Grinding, decoction; Taken orally for jaundiced } \\
\text { hepatitis, fever, diarrhea, nephritis and edema }\end{array}$ \\
\hline 148 & Gastrodia elata Blume & Tianma天麻 & non ${ }^{5} \mathrm{bu}^{4} \mathrm{non}^{2}$ & Orchidaceae & Herb & Wild & Rhizome & $\begin{array}{l}\text { Grinding, decoction; Taken orally for headache } \\
\text { and stomachache }\end{array}$ \\
\hline
\end{tabular}


Table 1 Inventory of Medicinal Plants Traditionally Used by Maonan People (Continued)

\begin{tabular}{|c|c|c|c|c|c|c|c|c|}
\hline 149 & $\begin{array}{l}\text { Gaultheria leucocarpa var. } \\
\text { yunnanensis (Franch.) T.Z. } \\
\text { Hsu \& R.C. Fang }\end{array}$ & Baiguobaizhu白果白珠 & $m e^{5} 7 a^{5}$ & Ericaceae & Shrub & Wild & Whole plant, Root & $\begin{array}{l}\text { Grinding, decoction; Taken orally for rheumatic } \\
\text { arthritis and traumatic injury }\end{array}$ \\
\hline 150 & $\begin{array}{l}\text { Gelsemium elegans } \\
\text { (Gardner \& Champ.) Benth. }\end{array}$ & Gouwen钩吻 & ruon $^{2} \mathrm{sai}^{3} \mathrm{mu}{ }^{5}$ & Loganiaceae & Liana & Wild & Whole plant & $\begin{array}{l}\text { Pound fresh part applied on the affected area, } \\
\text { treating for furuncle, carbuncle }\end{array}$ \\
\hline 151 & Gentiana rhodantha Franch. & $\begin{array}{l}\text { Honghualongdan红花 } \\
\text { 龙胆 }\end{array}$ & $y a^{5} m a^{2} m ə n p^{8}$ & Gentianaceae & Herb & Wild & Root & Grinding, decoction; Taken orally for furuncle \\
\hline 152 & Geranium nepalense Sweet & $\begin{array}{l}\text { Nibo'er'laoguancao尼泊 } \\
\text { 尔老鹳草. }\end{array}$ & $m a^{6} z a n^{5} n a n^{5}$ & Geraniaceae & Herb & Wild & Whole plant & $\begin{array}{l}\text { Grinding, decoction; Taken orally for pertussis } \\
\text { cough }\end{array}$ \\
\hline 153 & $\begin{array}{l}\text { Gerbera piloselloides } \\
\text { (L.) Cass. }\end{array}$ & $\begin{array}{l}\text { Maodadingcao毛大丁 } \\
\text { 草 }\end{array}$ & ruon təp $^{7}$ thi $^{6}$ & Asteraceae & Herb & Wild & Whole plant & $\begin{array}{l}\text { Grinding, decoction; Taken orally for clearing } \\
\text { away heat and toxic materials, fever due to } \\
\text { common cold, cough, diarrhea, infantile } \\
\text { indigestion }\end{array}$ \\
\hline 154 & Geum aleppicum Jacq. & Lubianqing路边青 & $m b a^{3} m_{e n}^{2}$ & Rosaceae & Herb & Wild & Whole plant & $\begin{array}{l}\text { Boiled with meat and drunk the soup, treating } \\
\text { for deficiency of dizziness }\end{array}$ \\
\hline 155 & Ginkgo biloba L. & Yinxing银杏 & - & $\begin{array}{l}\text { Ginkgoaceae } \\
\text { Engler }\end{array}$ & Tree & Homegarden & Fruit, Leaf & $\begin{array}{l}\text { Grinding, decoction; Taken orally for moistening } \\
\text { lung, cough }\end{array}$ \\
\hline 156 & Gleditsia sinensis Lam. & Zaojia㿝荚 & - & Fabaceae & Tree & Both & Pod & $\begin{array}{l}\text { Grinding, decoction; Taken orally for apocenosis, } \\
\text { detumescence }\end{array}$ \\
\hline 157 & $\begin{array}{l}\text { Glochidion puberum } \\
\text { (Linnaeus) Hutchinson }\end{array}$ & Suanpanzi算盘子 & $m e i^{4} \operatorname{th}^{6} \operatorname{ten}^{5}$ & Euphorbiaceae & Shrub & Wild & Root, Leaf & $\begin{array}{l}\text { Grinding, decoction; Taken orally for bacillary } \\
\text { diarrhea, infantile indigestion, diarrhea, } \\
\text { abdomen pain, proctoptosis, migraine, } \\
\text { lymphnoditis }\end{array}$ \\
\hline 158 & Gomphrena globosa L. & Qianrihong千日红 & $x i e n^{3} v^{3} n^{3} \mid a n^{1}$ & Amaranthaceae & Herb & Wild & Flower & $\begin{array}{l}\text { Grinding, decoction; Taken orally for asthma, } \\
\text { bronchitis, pertussis cough, tuberculosis, } \\
\text { diarrhea and hemoptysis }\end{array}$ \\
\hline 159 & $\begin{array}{l}\text { Gonostegia hirta } \\
\text { (Blume ex Hassk.) Miq. }\end{array}$ & Nuomituan糯米团 & - & Urticaceae & Herb & Wild & Whole plant, Root & $\begin{array}{l}\text { Grinding, decoction or boiled with meat and } \\
\text { drunk for clearing away heat and removing } \\
\text { dampness, innominate inflanunatory of } \\
\text { unknown origin }\end{array}$ \\
\hline 160 & $\begin{array}{l}\text { Gymnotheca chinensis } \\
\text { Decne. }\end{array}$ & Luoshuo裸䕛 & $\operatorname{man}^{5} w \varepsilon \eta^{3} b o a^{5}$ & Saururaceae & Herb & Homegarden & Whole plant & $\begin{array}{l}\text { Boiled with meat and drunk the soup, treating } \\
\text { for weakness and cough }\end{array}$ \\
\hline 161 & $\begin{array}{l}\text { Gynostemma pentaphyllum } \\
\text { (Thunb.) Makino }\end{array}$ & Jiaogulan绞股蓝 & - & Cucurbitaceae & Liana & Wild & Whole plant & $\begin{array}{l}\text { Grinding, decoction; Taken orally for rheumatism, } \\
\text { bronchitis and stomachache }\end{array}$ \\
\hline 162 & Hedyotis diffusa Willd. & $\begin{array}{l}\text { Baihuasheshecao白花蛇 } \\
\text { 舌草. }\end{array}$ & ruon ${ }^{2} m a^{2} r u i^{2} s \varepsilon^{5}$ & Rubiaceae & Herb & Wild & Whole plant & $\begin{array}{l}\text { Grinding, decoction; Taken orally for hepatitis, } \\
\text { cough, bronchitis, tonsillitis }\end{array}$ \\
\hline 163 & $\begin{array}{l}\text { Hemsleya sphaerocarpa } \\
\text { Kuang \& A. M. Lu }\end{array}$ & Shelian蛇莲 & tei 7 na: $^{2}$ & Cucurbitaceae & Liana & Wild & Tuber & Powdered; Taken orally for appendicitis \\
\hline 164 & $\begin{array}{l}\text { Homalomena occulta } \\
\text { (Lour.) Schott }\end{array}$ & Qiannianjian千年健 & $m a^{6} \mathrm{mon}^{3} \mathrm{t} \epsilon \varepsilon^{5}$ & Araceae & Herb & Wild & Rhizome & $\begin{array}{l}\text { Grinding, decoction; Taken orally for rheumatism, } \\
\text { numbness of limbs, traumatic injury, bone } \\
\text { fracture }\end{array}$ \\
\hline
\end{tabular}


Table 1 Inventory of Medicinal Plants Traditionally Used by Maonan People (Continued)

\begin{tabular}{|c|c|c|c|c|c|c|c|c|}
\hline$\overline{165}$ & Houttuynia cordata Thunb. & Yuxingcao鱼腥草 & $\mathrm{mba}^{3} \mathrm{wət}^{8}$ & Saururaceae & Herb & Homegarden & Whole plant & $\begin{array}{l}\text { Grinding, decoction; Taken orally for edema, } \\
\text { bronchopneumonia, nephritis, enteritis, } \\
\text { diarrhea, cough }\end{array}$ \\
\hline 166 & $\begin{array}{l}\text { Hydrocotyle nepalensis } \\
\text { Hook }\end{array}$ & Hongmaticao红马蹄草 & - & Umbelliferae & Herb & Wild & Whole plant & $\begin{array}{l}\text { Pound fresh part mixing with hot liquor and } \\
\text { applied on the affected area, treating for } \\
\text { traumatic injury }\end{array}$ \\
\hline 167 & $\begin{array}{l}\text { Hydrocotyle } \\
\text { sibthorpioides Lam. }\end{array}$ & Tianhusui天胡荌 & $n a^{5} \operatorname{tgiao}{ }^{2} n \varepsilon m^{3}$ & Umbelliferae & Herb & Wild & Whole plant & $\begin{array}{l}\text { Grinding, decoction; Taken orally for headache } \\
\text { due to common cold }\end{array}$ \\
\hline 168 & $\begin{array}{l}\text { Hypericum japonicum } \\
\text { Thunb. }\end{array}$ & Tianjihuang田基黄 & ruon $^{2} \mathrm{kha}^{3} \mathrm{kai}^{5}$ & Guttiferae & Herb & Wild & Whole plant & $\begin{array}{l}\text { Grinding, decoction; Taken orally for hepatitis, } \\
\text { acute conjunctivitis, tonsillitis and forepart } \\
\text { hepatocirrhosis }\end{array}$ \\
\hline 169 & $\begin{array}{l}\text { Hypericum sampsonii } \\
\text { Hance }\end{array}$ & Yuanbaocao元宝草 & wa ${ }^{3} \operatorname{ci\varepsilon n}^{3}$ & Guttiferae & Herb & Wild & Whole plant & $\begin{array}{l}\text { Grinding, decoction; Taken orally for traumatic } \\
\text { injury, pain, indigestion, chest congestion }\end{array}$ \\
\hline 170 & $\begin{array}{l}\text { Illicium difengpi B.N. } \\
\text { Chang }\end{array}$ & Difengpi地枫皮 & - & Magnoliaceae & Shrub & Wild & Stem, Bark & $\begin{array}{l}\text { Grinding, decoction; Taken orally for rheumatism, } \\
\text { rheumatic arthralgia and lumbar muscle } \\
\text { degeneration }\end{array}$ \\
\hline 171 & Impatiens balsamina $\mathrm{L}$. & Fengxianhua 凤仙花 & $w a^{3} \mathrm{dip}^{7} \operatorname{sim}^{1}$ & Balsaminaceae & Herb & Homegarden & Whole plant, Seed & $\begin{array}{l}\text { Pound fresh part applied on the affected area, } \\
\text { treating for furuncle, carbuncle }\end{array}$ \\
\hline 172 & $\begin{array}{l}\text { Impatiens pinfanensis } \\
\text { Hook. f. }\end{array}$ & $\begin{array}{l}\text { Kuaijiefengxianhua块节 } \\
\text { 凤仙花 }\end{array}$ & $\operatorname{fan}^{4} m \varepsilon^{5} m a^{2}$ & Balsaminaceae & Herb & Homegarden & Tuber & $\begin{array}{l}\text { Pound fresh part applied on the affected area, } \\
\text { treating for scrofula }\end{array}$ \\
\hline 173 & $\begin{array}{l}\text { Imperata cylindrica } \\
\text { (L.) P. Beauv. }\end{array}$ & Baimao白茅 & $\tan ^{5} \mathrm{ya}^{3}$ guan $^{4}$ & Gramineae & Herb & Wild & Rhizome & $\begin{array}{l}\text { Grinding, decoction; Taken orally for nephritis, } \\
\text { edema, bleeding }\end{array}$ \\
\hline 174 & $\begin{array}{l}\text { Ipomoea cairica } \\
\text { (L.) Sweet }\end{array}$ & $\begin{array}{l}\text { Wuzhaojinlong五爪金 } \\
\text { 龙 }\end{array}$ & ruon $^{2} l a k^{8} o \eta^{5}$ & Convolvulaceae & Herb & Both & Leaf, Tuber & $\begin{array}{l}\text { Pound fresh part applied on the affected area, } \\
\text { treating for carbuncle, clearing away heat and } \\
\text { toxic materials }\end{array}$ \\
\hline 175 & $\begin{array}{l}\text { Ipomoea mauritiana } \\
\text { Jacq. }\end{array}$ & Qizhualong七爪龙 & miau $^{2} r n^{3} \operatorname{sen}^{5}$ & Convolvulaceae & Liana & Both & Tuber, Leaf & $\begin{array}{l}\text { Boiled with meat and drunk the soup, treating } \\
\text { for nephritis }\end{array}$ \\
\hline 176 & $\begin{array}{l}\text { Ipomoea pescaprae } \\
\text { (L.) R. Br. }\end{array}$ & Houteng厚藤 & ruon $^{2} a^{3} \mathrm{mia}^{4}$ & Convolvulaceae & Herb & Wild & Whole plant & $\begin{array}{l}\text { Grinding, decoction; Taken orally for rheumatic } \\
\text { lumbocrural pain and lumbar muscle } \\
\text { degeneration }\end{array}$ \\
\hline 177 & Iris tectorum Maxim & Yuanwei䒽尾 & zo $0^{5}$ wa:n $n^{1}$ & Iridaceae & Herb & Both & Rhizome & $\begin{array}{l}\text { Pound fresh part with water is taken as a drink } \\
\text { for improving indigestion }\end{array}$ \\
\hline 178 & Juglans regia $\mathrm{L}$. & Hutao胡桃 & den $^{3} \operatorname{van}^{5} k \partial \eta^{2}$ & Juglandaceae & Tree & Both & Fruit & $\begin{array}{l}\text { Grinding, decoction; Taken orally for tonic, } \\
\text { back pain }\end{array}$ \\
\hline 179 & Juncus effusus L. & Dengxincao灯心草 & $n_{6} a n^{6} d a: n^{5}$ & Juncaceae & Herb & Wild & Whole plant & $\begin{array}{l}\text { Grinding, decoction; Taken orally for jaundiced } \\
\text { hepatitis }\end{array}$ \\
\hline 180 & $\begin{array}{l}\text { Justicia gendarussa } \\
\text { Burm. f. }\end{array}$ & Xiaobogu小驳骨 & ruon $^{2}$ tiək $^{7} d a k^{8} s \varepsilon^{5}$ & Acanthaceae & Shrub & Wild & Stem, Leaf & $\begin{array}{l}\text { Pound fresh part applied on the affected area, } \\
\text { treating for bone fracture, traumatic injury, } \\
\text { 2rheumatic arthritis, ulcer }\end{array}$ \\
\hline 181 & $\begin{array}{l}\text { Justicia ventricosa Wall. } \\
\text { ex Hook. f. }\end{array}$ & $\begin{array}{l}\text { Heiyexiaobogu黑叶小 } \\
\text { 驳骨 }\end{array}$ & - & Acanthaceae & Shrub & Wild & Stem, Leaf & $\begin{array}{l}\text { Pound fresh part applied on the affected area, } \\
\text { treating for bone fracture, traumatic injury, } \\
\text { rheumatic arthritis, waist pain, bleeding }\end{array}$ \\
\hline
\end{tabular}


Table 1 Inventory of Medicinal Plants Traditionally Used by Maonan People (Continued)

\begin{tabular}{|c|c|c|c|c|c|c|c|c|}
\hline$\overline{182}$ & $\begin{array}{l}\text { Kadsura heteroclita (Roxb.) } \\
\text { Craib }\end{array}$ & $\begin{array}{l}\text { Yixingnanwuweizi异形 } \\
\text { 南五味子 }\end{array}$ & ruon ${ }^{2} i^{5} r w p^{8}$ & Magnoliaceae & Liana & Wild & Stem & $\begin{array}{l}\text { Grinding, decoction or infusion with wine } \\
\text { drunk for bone fracture, ostealgia, chronic } \\
\text { gastricism, acute gastroenteritis }\end{array}$ \\
\hline 183 & $\begin{array}{l}\text { Kadsura } \\
\text { longipedunculata } \\
\text { Finet \& Gagnep. }\end{array}$ & Nanwuweizi南五味子 & - & Magnoliaceae & Liana & Wild & Fruit & Decoctionn; Taken orally for cough, insomnia \\
\hline 184 & $\begin{array}{l}\text { Kalimeris indica } \\
\text { (L.) Sch. Bip. }\end{array}$ & Malan马兰 & ruon $^{2} \operatorname{xien}^{3} \mathrm{sok}^{7}$ & Asteraceae & Herb & Wild & Whole plant & $\begin{array}{l}\text { Grinding, decoction; Taken orally for pneumonia, } \\
\text { bronchitis }\end{array}$ \\
\hline 185 & $\begin{array}{l}\text { Kyllinga brevifolia } \\
\text { Rottb. }\end{array}$ & $\begin{array}{l}\text { Duanyeshuiwugong短 } \\
\text { 叶水蜈蚣 }\end{array}$ & - & Cyperaceae & Herb & Wild & Whole plant & $\begin{array}{l}\text { Grinding, decoction; Taken orally for infantile } \\
\text { malnutrition, helminth }\end{array}$ \\
\hline 186 & $\begin{array}{l}\text { Laggera alata } \\
\text { (D. Don) Sch. Bip. } \\
\text { ex Oliv. }\end{array}$ & Liulengju六棱菊 & $r^{\prime u o n}{ }^{2} j \varepsilon n^{3} n \partial m^{1}$ & Asteraceae & Herb & Wild & Whole plant & $\begin{array}{l}\text { Grinding, decoction; Taken orally for rheumatic } \\
\text { arthritis, nephritis, edema }\end{array}$ \\
\hline 187 & $\begin{array}{l}\text { Laportea cuspidata } \\
\text { (Wedd.) Friis }\end{array}$ & Aima艾麻 & tuom ${ }^{2} r \varepsilon n^{5}$ & Urticaceae & Herb & Wild & Whole plant, Root & Medicinal liquor for rheumatic arthritis \\
\hline 188 & $\begin{array}{l}\text { Leonurus artemisia } \\
\text { (Lour.) S.Y. Hu }\end{array}$ & Yimucao益母草 & $\mathrm{ra}^{2} \mid \operatorname{lon}^{2} \mathrm{cit}^{7} v \partial n^{6}$ & Lamiaceae & Herb & Wild & Whole plant & $\begin{array}{l}\text { Grinding, decoction; Taken orally for edema, } \\
\text { nephritis, abnormal menstruation, promoting } \\
\text { blood circulation due to menstruation }\end{array}$ \\
\hline 189 & $\begin{array}{l}\text { Ligusticum chuanxiong } \\
\text { S.H. Qiu, Y.Q. Zeng, K.Y. Pan, } \\
\text { Y.C. Tang \& J.M. Xu }\end{array}$ & Chuanxiong川芦 & $\operatorname{ta}^{5}$ chuan $^{2} w o \eta^{5}$ & Umbelliferae & Herb & Both & Rhizome & $\begin{array}{l}\text { Grinding, decoction; Taken orally for carminative, } \\
\text { activate blood for acesodyne }\end{array}$ \\
\hline 190 & Ligusticum sinense Oliv. & Gaoben㩰本 & - & Umbelliferae & Herb & Wild & Whole plant & Medicinal liquor for waist pain, kidney deficiency \\
\hline 191 & $\begin{array}{l}\text { Ligustrum lucidum } \\
\text { W.T. Aiton }\end{array}$ & Nüzhen女贞 & - & Oleaceae & Tree & Homegarden & Fruit & $\begin{array}{l}\text { Grinding, decoction; Taken orally for tonifying } \\
\text { kieney and liver }\end{array}$ \\
\hline 192 & $\begin{array}{l}\text { Ligustrum robustum subsp. } \\
\text { chinense P.S. Green }\end{array}$ & $\begin{array}{l}\text { Cuzhuangnüzhen粗壮 } \\
\text { 女贞 }\end{array}$ & va ${ }^{3}$ zhe $\varepsilon^{5} \mathrm{ganm}^{2}$ & Oleaceae & Tree & Homegarden & Leaf & Drink like the tea for dizziness \\
\hline 193 & $\begin{array}{l}\text { Lilium brownii F.E. } \\
\text { Brown ex Miellez }\end{array}$ & Yebaihe野百合 & $k \supset \eta^{1} d \jmath^{2} \mathrm{pa}^{5}$ & Liliaceae & Herb & Both & Bulb & $\begin{array}{l}\text { Grinding, decoction; Taken orally for tuberculosis, } \\
\text { edema, insomnia, neurasthenia and palpitation }\end{array}$ \\
\hline 194 & $\begin{array}{l}\text { Lindera aggregata } \\
\text { (Sims) Kosterm. }\end{array}$ & Wuyao乌药 & - & Lauraceae & Shrub & Wild & Root & $\begin{array}{l}\text { Grinding, decoction; Taken orally for } \\
\text { stomachache, abdomon pain }\end{array}$ \\
\hline 195 & Litchi chinensis Sonn. & Lizhi荔枝 & - & Sapindaceae & Tree & Homegarden & Stone fruit & $\begin{array}{l}\text { Grinding, decoction; Taken orally for rheumatism, } \\
\text { pain and removing moping }\end{array}$ \\
\hline 196 & $\begin{array}{l}\text { Lithospermum erythrorhizon } \\
\text { Siebold } \\
\text { \& Zucc. }\end{array}$ & Zicao紫草 & gan $^{5}$ pat $^{8}$ & Boraginaceae & Herb & Wild & Root & Grinding, decoction; Taken orally for measles \\
\hline 197 & $\begin{array}{l}\text { Litsea cubeba (Lour.) } \\
\text { Pers. }\end{array}$ & Shanjijiao 山鸡椒 & - & Lauraceae & Shrub & Both & Fruit & $\begin{array}{l}\text { Pound fresh/dry fruit, decoction; Taken orally } \\
\text { for cough, diarrhea, stomachache, toothache, } \\
\text { bleeding }\end{array}$ \\
\hline 198 & Litsea pungens Hemsl. & Mujiangzi木姜子 & ruon $^{2} m e i^{4} \operatorname{san}^{1}$ & Lauraceae & Tree & Both & Root & Grinding, decoction; Taken orally for gastricism \\
\hline 199 & Livistona chinensis & Pukui蒲葵 & ruon $^{2} x i e n^{5}$ phu $^{2}$ & Palmae & Tree & Homegarden & Seed & Grinding, decoction; Taken orally for cancer \\
\hline
\end{tabular}


Table 1 Inventory of Medicinal Plants Traditionally Used by Maonan People (Continued)

\begin{tabular}{|c|c|c|c|c|c|c|c|c|}
\hline 200 & Lobelia chinensis Lour. & Banbianlian半边莲 & nun $^{3} \mathrm{mua}^{2} 2 \mathrm{n \varepsilon m} \mathrm{m}^{4}$ & Campanulaceae & Herb & Wild & Whole plant & $\begin{array}{l}\text { Grinding, boiled with water and washed the } \\
\text { affected area for snake bite }\end{array}$ \\
\hline 201 & $\begin{array}{l}\text { Lobelia sequinii } \\
\text { Levl. et Vant. }\end{array}$ & $\begin{array}{l}\text { Xi'nanshan'gengcai西南 } \\
\text { 山梗菜 }\end{array}$ & ruon$^{2}$ thai $^{6} \operatorname{tscn}^{1} \mathrm{cuwn}{ }^{3}$ & Campanulaceae & Herb & Wild & Whole plant & $\begin{array}{l}\text { Grinding, boiled with water and washed the } \\
\text { affected area for rheumatic arthritis, traumatic } \\
\text { injury, ulcer }\end{array}$ \\
\hline 202 & $\begin{array}{l}\text { Lonicera japonica } \\
\text { Thunb. }\end{array}$ & Rendong忍冬 & $w a^{3} \operatorname{cim}^{3} \operatorname{mon}^{2}$ & Caprifoliaceae & Liana & Both & Stem, Flower & $\begin{array}{l}\text { Grinding and decoction; Taken orally; Stem is } \\
\text { for jaundice, clearing away heat and toxic } \\
\text { materials, headache and fever; flower is for } \\
\text { enteritis, diarrhea, pneumonia, influenza }\end{array}$ \\
\hline 203 & $\begin{array}{l}\text { Lophatherum gracile } \\
\text { Brongn. }\end{array}$ & Danzhuye淡竹叶 & $m e i^{4} \operatorname{tim}^{1} s \varepsilon^{5}$ & Gramineae & Herb & Both & Leaf & $\begin{array}{l}\text { Grinding, decoction; Taken orally for urinary } \\
\text { tract infection, aphthous stomatitis, swelling, } \\
\text { aching of gum }\end{array}$ \\
\hline 204 & $\begin{array}{l}\text { Loropetalum chinense } \\
\text { (R. Br.) Oliv. }\end{array}$ & Jimu聯木 & ruon ${ }^{2} m e^{4}{ }^{4} i^{5}$ & Hamamelidaceae & Shrub & Wild & Leaf, Flower, Root & $\begin{array}{l}\text { Grinding, decoction; Taken orally; Leaf is for } \\
\text { abdomen pain, metrorrhagia; Flower is for } \\
\text { bleeding; Root is for traumatic injury, chronic } \\
\text { arthritis, amenorrhea, bleeding }\end{array}$ \\
\hline 205 & $\begin{array}{l}\text { Lycopodium japonicum } \\
\text { Thunb. }\end{array}$ & Shisong石松 & mlau $^{2}$ muan $^{4}$ & Lycopodiaceae & - & Wild & Whole plant & $\begin{array}{l}\text { Grinding, decoction; Taken orally for rheumatic } \\
\text { arthritis, arthralgia, leg cramp, hand and foot } \\
\text { numbness }\end{array}$ \\
\hline 206 & $\begin{array}{l}\text { Lycopus lucidus Turcz. } \\
\text { ex Benth. }\end{array}$ & Disun地笋 & - & Lamiaceae & Herb & Wild & Whole plant & $\begin{array}{l}\text { Grinding, decoction; Taken orally for abnormal } \\
\text { menstruation, amenorrhea, traumatic injury, } \\
\text { bone fracture }\end{array}$ \\
\hline 207 & $\begin{array}{l}\text { Lygodium japonicum } \\
\text { (Thunb.) Sw. }\end{array}$ & Haijinsha海金沙 & $m a^{2} g o n^{2} \mathrm{bou}^{3}$ & Lygodiaceae & - & Wild & $\begin{array}{l}\text { Whole plant, } \\
\text { Sporangium }\end{array}$ & $\begin{array}{l}\text { Pound fresh part applied on the affected area, } \\
\text { treating for chronic ulcer, skin infection, } \\
\text { furuncle, foot rot }\end{array}$ \\
\hline 208 & $\begin{array}{l}\text { Lysimachia christinae } \\
\text { Hance }\end{array}$ & Guoluhuang过路黄 & $m a^{6} g 7 o u^{2} 7 a n^{2}$ & Primulaceae & Herb & Wild & Whole plant & $\begin{array}{l}\text { Grinding, decoction; Taken orally for urinary } \\
\text { tract infection, jaundice, hepatitis }\end{array}$ \\
\hline 209 & $\begin{array}{l}\text { Lysimachia paridiformis } \\
\text { Franch. }\end{array}$ & Luodimei落地梅 & - & Primulaceae & Herb & Wild & Whole plant & $\begin{array}{l}\text { Grinding, decoction; Taken orally for infantile } \\
\text { convulsions }\end{array}$ \\
\hline 210 & $\begin{array}{l}\text { Lysionotus pauciflorus } \\
\text { Maxim. }\end{array}$ & Diaoshijutai吊石苣苔 & $b a^{5} d a u^{3} m a^{4}$ & Gesneriaceae & Shrub & Wild & Whole plant & $\begin{array}{l}\text { Grinding, decoction; Taken orally for bronchitis, } \\
\text { asthma }\end{array}$ \\
\hline 211 & $\begin{array}{l}\text { Magnolia officinalis } \\
\text { Rehder \& E.H. Wilson }\end{array}$ & Houpo厚朴 & - & Magnoliaceae & Tree & Both & Bark & $\begin{array}{l}\text { Grinding, decoction; Taken orally for emesis, } \\
\text { diarrhea }\end{array}$ \\
\hline 212 & $\begin{array}{l}\text { Mahonia bealei } \\
\text { (Fortune) Carrière }\end{array}$ & $\begin{array}{l}\text { Kuoyeshidagonglao阔 } \\
\text { 叶十大功劳 }\end{array}$ & ruon $^{2}$ wan $^{6}{ }^{6}$ ien $^{4}$ & Berberidaceae & Shrub & Wild & Root & $\begin{array}{l}\text { Grinding, decoction; Taken orally for pneumonia, } \\
\text { tuberculosis, infectious hepatitis, acute } \\
\text { gastroenteritis, bronchitis }\end{array}$ \\
\hline 213 & $\begin{array}{l}\text { Mallotus apelta } \\
\text { (Lour.) Müll. Arg. }\end{array}$ & Baibeiye白背叶 & mei $^{4}$ phiau $^{6}$ sei $^{1}$ & Euphorbiaceae & Shrub & Wild & Root, Leaf & $\begin{array}{l}\text { Grinding, decoction; Taken orally; Root is for } \\
\text { chronic hepatitis, hepatosplenomegaly, } \\
\text { gestational edema, enteritis, diarrhea; Leaf } \\
\text { is for traumatic injury, otitis media, furuncle, } \\
\text { bleeding, thrush }\end{array}$ \\
\hline 214 & $\begin{array}{l}\text { Mallotus barbatus } \\
\text { Müll. Arg. }\end{array}$ & Maotong毛桐 & - & Euphorbiaceae & Shrub & Wild & Leaf & $\begin{array}{l}\text { Pound fresh part and applied on the affected } \\
\text { area, treating for clearing away heat and toxic } \\
\text { materials, bed ulcer, eczema }\end{array}$ \\
\hline
\end{tabular}


Table 1 Inventory of Medicinal Plants Traditionally Used by Maonan People (Continued)

\begin{tabular}{|c|c|c|c|c|c|c|c|c|}
\hline 215 & Marsilea quadrifolia $\mathrm{L}$. & Ping苹 & phun $^{6}$ phieu $^{2} l a u^{4}$ & Marsileaceae & - & Wild & Whole plant & $\begin{array}{l}\text { Grinding, decoction; Taken orally for jaundiced } \\
\text { hepatitis, asthma, edema, hepatic ascites, fever } \\
\text { due to common cold }\end{array}$ \\
\hline 216 & $\begin{array}{l}\text { Melastoma candidum } \\
\text { D. Don }\end{array}$ & Yemudan野牡丹 & ruon $^{2} l a k^{8} \mathrm{ma}^{5} \mathrm{ndi}^{5}$ & Melastomataceae & Shrub & Wild & Whole plant & $\begin{array}{l}\text { Grinding, decoction; Taken orally for hemafecia, } \\
\text { watery diarrhea }\end{array}$ \\
\hline 217 & $\begin{array}{l}\text { Melastoma } \\
\text { dodecandrum Lour. }\end{array}$ & Dinie地菍 & $\operatorname{lak}^{8} \mathrm{nin}^{1}$ & Melastomataceae & Shrub & Wild & Whole plant & $\begin{array}{l}\text { Grinding, decoction; Taken orally for removing } \\
\text { blood stasis, traumatic injury, diarrhea, } \\
\text { lithangiuria, urinary obstruction }\end{array}$ \\
\hline 218 & Melia azedarach L. & Lian楝 & ruon $^{2} \mathrm{ku}^{1} \mathrm{lien}^{4}$ & Meliaceae & Tree & Both & Fruit, Leaf & $\begin{array}{l}\text { Grinding, boiled with water and washed the } \\
\text { affected area for scabies, tinea capitis and rice } \\
\text { paddies dermatitis }\end{array}$ \\
\hline 219 & Mimosa pudica $\mathrm{L}$. & Hanxiucao含羞草 & ruon $^{2} \mathrm{ra}^{2} n n \mathrm{i}^{3}$ & Fabaceae & Herb & Both & Whole plant & Grinding, decoction; Taken orally for insomnia \\
\hline 220 & Mirabilis jalapa L. & Zimol橴莱莉 & $r^{\prime} u n^{2} j \varepsilon n^{3} w a^{3}$ & Nyctaginaceae & Herb & Both & Root & $\begin{array}{l}\text { Boiled with meat and drunk the soup, treating } \\
\text { for leucorrhoea, abnormal menstruation, } \\
\text { prostatitis, metrorrhagia }\end{array}$ \\
\hline 221 & $\begin{array}{l}\text { Momordica cochinchinensis } \\
\text { (Lour.) Spreng. }\end{array}$ & Mubiezi木鳖子 & $\operatorname{tin}^{5} \mathrm{ndin}^{5} \mathrm{ka} \mathrm{n}^{3}$ & Cucurbitaceae & Liana & Wild & Seed, Leaf, Stem & $\begin{array}{l}\text { Grinding, decoction; Taken orally for innominate } \\
\text { inflanunatory of unknown origin, carbuncle, } \\
\text { lymphnoditis }\end{array}$ \\
\hline 222 & Morus alba L. & Sang桑 & ruon'tshan' & Moraceae & Tree & Both & Leaf, Bark & $\begin{array}{l}\text { Grinding, decoction; Taken orally for lung heat } \\
\text { panting and cough, hypertension, edema }\end{array}$ \\
\hline 223 & $\begin{array}{l}\text { Munronia henryi } \\
\text { Harms }\end{array}$ & Aituotuo矮陀陀 & - & Meliaceae & Shrub & Wild & Whole plant & Medicinal liquor for traumatic injury \\
\hline 224 & Murraya exotica L. & Jiulixiang九里香 & ruon $^{2} m i^{4} n d a n^{3}$ & Rutaceae & Tree & Both & Root, Leaf & $\begin{array}{l}\text { Grinding, decoction; Taken orally for rheumatism, } \\
\text { ostealgia, traumatic injury, toothache and } \\
\text { stomachache }\end{array}$ \\
\hline 225 & $\begin{array}{l}\text { Mussaenda pubescens W.T. } \\
\text { Aiton }\end{array}$ & Yuyejinhua玉叶金花 & ruon $^{2}$ phi $^{3} v^{5}$ phuok $^{8}$ & Rubiaceae & Shrub & Both & Stem, Leaf & $\begin{array}{l}\text { Grinding, decoction; Taken orally for } \\
\text { hyperthermia, influenza, tonsillitis, enteritis, } \\
\text { diarrhea and sphagitis }\end{array}$ \\
\hline 226 & $\begin{array}{l}\text { Myrica rubra (Lour.) Siebold } \\
\text { \& Zucc. }\end{array}$ & Yangmei杨梅 & $\operatorname{lak}^{8} \mathrm{se}^{5}$ & Myricaceae & Tree & Homegarden & Root Bark & $\begin{array}{l}\text { Grinding, decoction; Taken orally for traumatic } \\
\text { injury, bone fracture, diarrhea, stomach and } \\
\text { duodenal ulcer }\end{array}$ \\
\hline 227 & Nandina domestica Thunb. & Nantianzhu南天竹 & $w a \eta^{6} l i \varepsilon n^{4} s \varepsilon^{5}$ & Berberidaceae & Shrub & Wild & Root, Stem, Fruit & $\begin{array}{l}\text { Grinding, decoction; Taken orally; Root and stem } \\
\text { are for cough, fever, conjunctivitis, diarrhea, } \\
\text { jaundice, hepatitis, traumatic injury. Fruit is for } \\
\text { cough, asthma, pertussis }\end{array}$ \\
\hline 228 & Nepeta cataria L. & Jingjie荆芥 & - & Lamiaceae & Herb & Homegarden & Whole plant & $\begin{array}{l}\text { Grinding, decoction; Taken orally for common } \\
\text { cold }\end{array}$ \\
\hline 229 & $\begin{array}{l}\text { Nephrolepis cordifolia } \\
\text { (L.) C. Presl }\end{array}$ & Shenjue肾硕 & $\operatorname{lak}^{8} n \partial n^{4}$ & Davalliaceae & - & Wild & $\begin{array}{l}\text { Rhizome, Leaf, } \\
\text { Whole plant }\end{array}$ & $\begin{array}{l}\text { Grinding, decoction; Taken orally for fever due } \\
\text { to common cold, cough, diarrhea, acute enteritis, } \\
\text { jaundiced hepatitis }\end{array}$ \\
\hline 230 & Oenanthe javanica (Blume) & Shuiqin水芹 & ma: ${ }^{6}$ toip ${ }^{7} z_{a m}{ }^{5}$ & Umbelliferae & Herb & Wild & Whole plant & Grinding, decoction; Taken orally for hypertension \\
\hline
\end{tabular}


Table 1 Inventory of Medicinal Plants Traditionally Used by Maonan People (Continued)

\begin{tabular}{|c|c|c|c|c|c|c|c|c|}
\hline 231 & $\begin{array}{l}\text { Ophioglossum } \\
\text { reticulatum L. }\end{array}$ & $\begin{array}{l}\text { Xinyeping'erxiaocao心 } \\
\text { 叶瓶尔小草 }\end{array}$ & ruon $^{2} m a^{2} r u i^{2}$ & Ophioglossaceae & - & Wild & Whole plant & $\begin{array}{l}\text { Pound fresh part applied on the affected area, } \\
\text { treating for furuncle, snake bite and acute } \\
\text { conjunctivitis }\end{array}$ \\
\hline 232 & $\begin{array}{l}\text { Ophiopogon japonicus } \\
\text { (L. f.) Ker Gawl. }\end{array}$ & Maidong麦冬 & ruon $^{2} l a k^{8} j u^{3}$ & Liliaceae & Herb & Both & Tuber & $\begin{array}{l}\text { Grinding, decoction; Taken orally for chronic } \\
\text { bronchitis, cough }\end{array}$ \\
\hline 233 & $\begin{array}{l}\text { Opuntia stricta (Haw.) } \\
\text { Haw. var. dillenii (Ker-Gawl.) } \\
\text { Benson }\end{array}$ & Xianrenzhang仙人掌 & $m a^{2} m \omega m^{4}$ & Cactaceae & Shrub & Both & Stem & $\begin{array}{l}\text { Pound fresh part applied on the affected area, } \\
\text { treating for parotitis, carbuncle, empyrosis }\end{array}$ \\
\hline 234 & $\begin{array}{l}\text { Oroxylum indicum } \\
\text { (L. ) Kurz }\end{array}$ & Muhudie木蝴蝶 & $m e i^{4} \partial n^{3} \mathrm{eu}^{5}$ & Bignoniaceae & Tree & Homegarden & Bark, Seed & $\begin{array}{l}\text { Grinding, decoction; Taken orally for acute } \\
\text { bronchitis, tuberculosis, jaundiced hepatitis, } \\
\text { sore throat }\end{array}$ \\
\hline 235 & $\begin{array}{l}\text { Osbeckia opipara } \\
\text { C.Y. Wu \& C. Chen }\end{array}$ & Chaotianguan朝天罐 & - & Melastomataceae & Shrub & Wild & Root & $\begin{array}{l}\text { Boiled with meat and drunk the soup, treating } \\
\text { for tonic, bleeding, diarrhea }\end{array}$ \\
\hline 236 & Oxalis corniculata $\mathrm{L}$. & Cujiangcao酢浆草 & $m^{3} a^{3}$ thwm sou $^{1}$ & Oxalidaceae & Herb & Wild & Whole plant & $\begin{array}{l}\text { Pound fresh part applied on the affected area, } \\
\text { treating for febrile convulsion, enteritis, diarrhea, } \\
\text { parotitis }\end{array}$ \\
\hline 237 & $\begin{array}{l}\text { Paederia scandens } \\
\text { (Lour.) Merr. }\end{array}$ & Jishiteng鸡矢藤 & $b i e u^{3} t \omega t^{7} m a^{3}$ & Rubiaceae & Liana & Wild & Whole plant, Root & $\begin{array}{l}\text { Medicinal liquor for treating flu, cough, pertussis } \\
\text { cough, diarrhea, stomachache, chest stuffiness }\end{array}$ \\
\hline 238 & Paeonia lactiflora Pall. & Shaoyao莐药 & - & Ranunculaceae & Herb & Homegarden & Root & Powder tied for pain and blood stasis \\
\hline 239 & Paeonia suffruticosa Andrew & Mudan牡丹 & ma $^{5}$ muan $^{4}$ & Ranunculaceae & Shrub & Homegarden & Root Bark & $\begin{array}{l}\text { Grinding, decoction; Taken orally for lobar } \\
\text { pneumonia }\end{array}$ \\
\hline 240 & $\begin{array}{l}\text { Palhinhaea cernua } \\
\text { (L.) Vasc. \& Franco }\end{array}$ & $\begin{array}{l}\text { Chuisuishisong垂穗石 } \\
\text { 松 }\end{array}$ & - & Lycopodiaceae & - & Wild & Whole plant & $\begin{array}{l}\text { Grinding, decoction; Taken orally for relaxing } \\
\text { tendons and activating collaterals, carminative, } \\
\text { blood stasis, bleeding }\end{array}$ \\
\hline 241 & Paris polyphylla Sm. & $\begin{array}{l}\text { Qiyeyizhihua七叶一枝 } \\
\text { 花 }\end{array}$ & $w a^{6} \operatorname{ten}^{5} \mathrm{va}^{1}$ & Trilliaceae & Herb & Wild & Whole plant & $\begin{array}{l}\text { Pound fresh part applied on the affected area, } \\
\text { treating for traumatic injury and snake bite }\end{array}$ \\
\hline 242 & $\begin{array}{l}\text { Pentasacme } \\
\text { championii Benth. }\end{array}$ & Shiluomo石萝藦 & ruon $^{2} \operatorname{san}^{3}$ nut $^{8}$ & Asclepiadaceae & Herb & Wild & Whole plant & $\begin{array}{l}\text { Grinding, decoction; Taken orally for rheumatism, } \\
\text { ostealgia, traumatic injury and ascites due to } \\
\text { cirrhosis; Pound fresh part applied on the } \\
\text { affected area, treating for snake bite, herpes } \\
\text { zosters }\end{array}$ \\
\hline 243 & $\begin{array}{l}\text { Perilla frutescens } \\
\text { (L.) Britton }\end{array}$ & Zisu紫苏 & $m b a^{3} h a^{5} \operatorname{lan}^{1}$ & Lamiaceae & Herb & Homegarden & Whole plant & $\begin{array}{l}\text { Grinding, decoction; Taken orally for common } \\
\text { cold, cough, asthma, emesis }\end{array}$ \\
\hline 244 & $\begin{array}{l}\text { Periploca forrestii } \\
\text { Schltr. }\end{array}$ & Heilonggu黑龙骨 & $m e i^{5} \mathrm{za}^{2} n a m^{5}$ & Asclepiadaceae & Shrub & Wild & Whole plant & Medicinal liquor for treating rheumatic arthritis \\
\hline 245 & $\begin{array}{l}\text { Phellodendron } \\
\text { amurense Rupr. }\end{array}$ & Huangbo黄檗 & $m e^{5} b \varepsilon^{2} 7 a n^{3}$ & Rutaceae & Tree & Wild & Bark & $\begin{array}{l}\text { Grinding, decoction; Taken orally for diabetes } \\
\text { insipidus }\end{array}$ \\
\hline 246 & Pholidota chinensis Lindl. & Shixiantao石仙桃 & ruon ${ }^{2} \times i e n^{3}$ thui $i^{2}$ & Orchidaceae & Herb & Wild & Whole plant & $\begin{array}{l}\text { Grinding, decoction; Taken orally for cough, } \\
\text { tuberculosis, scrofula, diuresis, infantile } \\
\text { malnutrition }\end{array}$ \\
\hline 247 & $\begin{array}{l}\text { Phragmites australis } \\
\text { (Cav.) Trin. ex Steud. }\end{array}$ & Luwei芦苇 & $\operatorname{gan}^{5} \mathrm{di}^{2} \mathrm{nau}^{5}$ & Gramineae & Herb & Wild & Root & $\begin{array}{l}\text { Grinding, decoction; Taken orally for infantile } \\
\text { whitish aphthae }\end{array}$ \\
\hline
\end{tabular}


Table 1 Inventory of Medicinal Plants Traditionally Used by Maonan People (Continued)

\begin{tabular}{|c|c|c|c|c|c|c|c|c|}
\hline 248 & Phyllanthus urinaria $\mathrm{L}$. & Yexiazhu叶下珠 & thun $^{6}$ thin $^{6}$ sei $^{1}$ & Euphorbiaceae & Herb & Wild & Whole plant & $\begin{array}{l}\text { Grinding, decoction; Taken orally for jaundiced } \\
\text { hepatitis, diarrhea, enteritis, nephritis, edema } \\
\text { and lithangiuria }\end{array}$ \\
\hline 249 & $\begin{array}{l}\text { Phyllodium pulchellum } \\
\text { (L.) Desv. }\end{array}$ & Paiqianshu排钱树 & ruon $^{2} v^{2} k^{8}$ rjen $^{2}$ & Fabaceae & Shrub & Wild & Leaf, Root & $\begin{array}{l}\text { Grinding, decoction; Taken orally for fever, } \\
\text { diarrhea, malaria, hepatitis, rheumatic ostealgia, } \\
\text { traumatic injury, schistosome }\end{array}$ \\
\hline 250 & Physalis angulata $\mathrm{L}$. & Kuzhi苦蘵 & - & Solanaceae & Herb & Wild & Whole plant & $\begin{array}{l}\text { Grinding, decoction; Taken orally for epidemic } \\
\text { parotitis, cough, jaundice, hepatitis, diarrhea }\end{array}$ \\
\hline 251 & $\begin{array}{l}\text { Phytolacca acinosa } \\
\text { Roxb. }\end{array}$ & Shanglu商陆 & $\left.\operatorname{lak}^{8} \mathrm{ph} \mathrm{k}^{8} \mathrm{don}\right)^{2}$ & Phytolaccaceae & Herb & Both & Root & $\begin{array}{l}\text { Grinding, decoction; Taken orally for cervical } \\
\text { erosion, digestibility ulcer, liver ascites, } \\
\text { constipation, diuresis }\end{array}$ \\
\hline 252 & Pilea cavaleriei H. Lév. & Shiyoucai石油菜 & bma ju'thui ${ }^{2}$ & Urticaceae & Herb & Wild & Whole plant & $\begin{array}{l}\text { Grinding, decoction; Taken orally for lung heat } \\
\text { panting, cough, tuberculosis, traumatic injury, } \\
\text { empyrosis, furuncle }\end{array}$ \\
\hline 253 & Piper hancei Maxim. & Shanju山蒟 & tshuon ${ }^{5}$ pifun $^{6}$ & Piperaceae & Liana & Wild & Stem, Leaf & $\begin{array}{l}\text { Grinding, decoction; Taken orally for lumbar } \\
\text { muscle degeneration, chronic gastricism, cough, } \\
\text { ostealgia, rheumatic arthritis, heatstroke, } \\
\text { numbness of limbs }\end{array}$ \\
\hline 254 & $\begin{array}{l}\text { Pistia stratiotes } \\
\text { Linnaeus Sp. }\end{array}$ & Dapiao大漂 & - & Araceae & Herb & Wild & Whole plant & $\begin{array}{l}\text { Pound fresh part applied on the affected area, } \\
\text { treating for removing blood stasis }\end{array}$ \\
\hline 255 & Plantago asiatica L. & Cheqian车前 & $m b a^{3} b o k^{8}$ & Plantaginaceae & Herb & Wild & Whole plant & $\begin{array}{l}\text { Grinding, decoction; Taken orally for urinary } \\
\text { tract infection, urinary stone, fever and cough } \\
\text { due to common cold, nephritis, edema, } \\
\text { bronchitis, hypertension }\end{array}$ \\
\hline 256 & $\begin{array}{l}\text { Platycodon grandiflorus } \\
\text { (Jacq.) A. DC. }\end{array}$ & Jiegeng桔梗 & - & Campanulaceae & Herb & Both & Root & $\begin{array}{l}\text { Grinding, decoction; Taken orally for } \\
\text { inflammation, cough }\end{array}$ \\
\hline 257 & Plumbago zeylanica L. & Baihuadan白花丹 & ruon $^{2} \mathrm{ra}^{2} \mathrm{vok}^{7}$ & Plumbaginaceae & Herb & Wild & Whole plant & $\begin{array}{l}\text { Grinding, decoction; Taken orally for traumatic } \\
\text { injury }\end{array}$ \\
\hline 258 & $\begin{array}{l}\text { Pogonia japonica } \\
\text { Rchb. f. }\end{array}$ & Zhulan朱兰 & $m a^{6} z a^{4} z a o^{2}$ & Orchidaceae & Herb & Wild & Whole plant & $\begin{array}{l}\text { Boiled with meat and drunk the soup, treating } \\
\text { for enuresis }\end{array}$ \\
\hline 259 & $\begin{array}{l}\text { Polygala japonica } \\
\text { Houtt. }\end{array}$ & Guazijin瓜子金 & ya $^{1} \mathrm{yin}^{4} z \varepsilon m^{2}$ & Polygalaceae & Herb & Wild & Whole plant & Grinding, decoction; Taken orally for neurasthenia \\
\hline 260 & $\begin{array}{l}\text { Polygonatum } \\
\text { cyrtonema Hua }\end{array}$ & $\begin{array}{l}\text { Duohuahuangjing多花 } \\
\text { 黄精 }\end{array}$ & $x \operatorname{in}^{2} z a^{2}$ & Liliaceae & Herb & Wild & Rhizome & $\begin{array}{l}\text { Pound fresh part mixed with rice wine, applied } \\
\text { on the affected area, treating for } \\
\text { lymphadenectasis }\end{array}$ \\
\hline 261 & $\begin{array}{l}\text { Polygonatum odoratum } \\
\text { (Mill.) Druce }\end{array}$ & Yuzhu玉竹 & - & Liliaceae & Herb & Homegarden & Rhizome & $\begin{array}{l}\text { Grinding, decoction; Taken orally for moistening } \\
\text { lung for rresting cough }\end{array}$ \\
\hline 262 & $\begin{array}{l}\text { Polygonatum sibiricum } \\
\text { Redouté }\end{array}$ & Huangjing黄精 & $r^{\prime u o n}{ }^{2} \sin ^{3} m \omega m n^{4}$ & Liliaceae & Herb & Both & Rhizome & $\begin{array}{l}\text { Grinding, decoction; Taken orally for tuberculosis, } \\
\text { diabetes, hypertension, weakness after ill, } \\
\text { invigorating spleen, reinforcing stomach }\end{array}$ \\
\hline 263 & Polygonum aviculare $\mathrm{L}$. & Shegan射干 & $\operatorname{lan}^{5} \mid u^{5} \mathrm{kun}^{2}$ & Polygonaceae & Herb & Wild & Whole plant & $\begin{array}{l}\text { Grinding, decoction; Taken orally for stranguria } \\
\text { due to hematuria }\end{array}$ \\
\hline
\end{tabular}


Table 1 Inventory of Medicinal Plants Traditionally Used by Maonan People (Continued)

\begin{tabular}{|c|c|c|c|c|c|c|c|c|}
\hline 264 & Polygonum chinense $\mathrm{L}$. & Huotanmu火炭母 & $v a^{5} \mathrm{mba}^{3} \mathrm{~s} \omega \mathrm{m}^{1}$ & Polygonaceae & Herb & Wild & $\begin{array}{l}\text { Whole plant, } \\
\text { Rhizome }\end{array}$ & $\begin{array}{l}\text { Grinding, decoction; Taken orally for diarrhea, } \\
\text { enteritis, indigestion, hepatitis, pharyngitis. } \\
\text { Pound fresh part applied on the affected area, } \\
\text { treating for traumatic injury, furuncle, eczema, } \\
\text { dermatitis, pruritus }\end{array}$ \\
\hline 265 & Polygonum hydropiper L. & Shuiliao辣蓼 & $m b a^{3} w e^{5}$ & Polygonaceae & Herb & Both & Whole plant & $\begin{array}{l}\text { Grinding, decoction; Taken orally for diarrhea, } \\
\text { acute ulcer, common cold, typhoid, rheumatism, } \\
\text { ostealgia, traumatic injury. Pound fresh part } \\
\text { applied on the affected area, treating for } \\
\text { eczema, centipede bite }\end{array}$ \\
\hline 266 & $\begin{array}{l}\text { Polygonum perfoliatum } \\
\text { (L.) L. }\end{array}$ & gangban'gui杜板归 & ruon $^{2} \operatorname{tin}^{3} \operatorname{di} k^{8}$ & Polygonaceae & Herb & Wild & Whole plant & $\begin{array}{l}\text { Grinding, decoction; Taken orally for jaundice, } \\
\text { diarrhea, malaria, nephritis, edema. Pound fresh } \\
\text { part applied on the affected area, treating for } \\
\text { furuncle, eczema, carbuncle }\end{array}$ \\
\hline 267 & Portulaca oleracea $\mathrm{L}$. & Machixian马齿苋 & ruon ${ }^{2} m b a^{3} n ә m^{1}$ & Portulacaceae & Herb & Wild & Whole plant & $\begin{array}{l}\text { Grinding, decoction; Taken orally for acute } \\
\text { cystitis, diarrhea, hypertension }\end{array}$ \\
\hline 268 & Potentilla chinensis Ser. & Weilingcai委陵菜 & $m a^{6} 7 \mathrm{gou}^{2} \mathrm{dui}{ }^{3}$ & Rosaceae & Herb & Wild & Whole plant & $\begin{array}{l}\text { Grinding, decoction; Taken orally for clearing } \\
\text { away heat and toxic materials, diarrhea }\end{array}$ \\
\hline 269 & Potentilla reyniana Bornm. & $\begin{array}{l}\text { Sanyeweilingcai三叶委 } \\
\text { 陵菜 }\end{array}$ & - & Rosaceae & Herb & Wild & Root & $\begin{array}{l}\text { Grinding, decoction; Taken orally for preventing } \\
\text { rabies }\end{array}$ \\
\hline 270 & $\begin{array}{l}\text { Potentilla kleiniana } \\
\text { Wight \& Arn. }\end{array}$ & $\begin{array}{l}\text { Shehanweilingcai蛇含 } \\
\text { 委陵菜 }\end{array}$ & 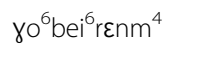 & Rosaceae & Herb & Wild & Whole plant & $\begin{array}{l}\text { Grinding the fresh part, decoction drunk for } \\
\text { infantile fever }\end{array}$ \\
\hline 271 & Prunella vulgaris $\mathrm{L}$. & Xiakucao夏枯草 & - & Lamiaceae & Herb & Wild & Whole plant & $\begin{array}{l}\text { Grinding, decoction; Taken orally for clearing } \\
\text { away heat and toxic materials }\end{array}$ \\
\hline 272 & Psoralea corylifolia L. & Buguzhi补骨脂 & - & Fabaceae & Herb & Wild & Seed & $\begin{array}{l}\text { Medicinal liquor for treating rheumatism and } \\
\text { kidney deficiency }\end{array}$ \\
\hline 273 & Pteris multifida Poir. & $\begin{array}{l}\text { Jinglanbiancao井栏边 } \\
\text { 草 }\end{array}$ & ruon $^{2} s \omega t^{7} k a i^{5}$ & Pteridaceae & - & Wild & Whole plant & $\begin{array}{l}\text { Grinding, decoction; Taken orally for diarrhea, } \\
\text { jaundiced hepatitis, hemafecia, hematuria }\end{array}$ \\
\hline 274 & $\begin{array}{l}\text { Pueraria lobata (Willd.) } \\
\text { Ohwi }\end{array}$ & Ge葛 & bieu $^{3} \mathrm{chai}^{5}$ & Fabaceae & Liana & Wild & Tuber & $\begin{array}{l}\text { Grinding, decoction; Taken orally for fever, } \\
\text { hypertension, protecting the liver, promoting } \\
\text { salivation }\end{array}$ \\
\hline 275 & $\begin{array}{l}\text { Pulsatilla chinensis (Bunge) } \\
\text { Regel }\end{array}$ & Baitouweng白头翁 & wok fian $^{3}$ puok $^{8}$ & Ranunculaceae & Herb & Wild & Rhizome & $\begin{array}{l}\text { Grinding, decoction; Taken orally for diarrhea, } \\
\text { malaria, dysmenorrhea, uterine bleeding }\end{array}$ \\
\hline 276 & Punica granatum L. & Shiliu石榴 & $\left|a k^{8}\right| i u^{2}$ & Punicaceae & Shrub & Homegarden & Pericarp & $\begin{array}{l}\text { Grinding, decoction; Taken orally for diarrhea, } \\
\text { acute enteritis, piles, proctoptosis }\end{array}$ \\
\hline 277 & Pyrola calliantha Andres & Luticao鹿蹄草 & - & Pyrolaceae & Herb & Wild & Whole plant & $\begin{array}{l}\text { Grinding, decoction; Taken orally for cough, } \\
\text { weakness }\end{array}$ \\
\hline 278 & $\begin{array}{l}\text { Pyrrosia lingua (Thunb.) } \\
\text { Farw. }\end{array}$ & Shiwei石韦 & $m b a^{3} m e i^{4} \mathrm{ri}^{2}$ & Polypodiaceae & - & Wild & Whole plant & $\begin{array}{l}\text { Grinding, decoction; Taken orally for senile } \\
\text { chronic bronchitis, pneumonia, nephritis, edema, } \\
\text { urinary tract infection }\end{array}$ \\
\hline 279 & Quisqualis indica $\mathrm{L}$. & Shijunzi使君子 & $1 a k^{6} r \partial m^{2}$ & Combretaceae & Liana & Wild & Seed & $\begin{array}{l}\text { Chewed for infantile malnutrition product, } \\
\text { depriving ascarid }\end{array}$ \\
\hline
\end{tabular}


Table 1 Inventory of Medicinal Plants Traditionally Used by Maonan People (Continued)

\begin{tabular}{|c|c|c|c|c|c|c|c|c|}
\hline 280 & $\begin{array}{l}\text { Rabdosia ternifolia (D. Don) } \\
\text { H. Hara }\end{array}$ & Niuweicao牛尾草 & $t \varepsilon^{5} \vee \varepsilon n^{3} \eta \eta^{2}$ & Lamiaceae & Herb & Wild & Whole plant, Leaf & $\begin{array}{l}\text { Grinding, decoction; Taken orally for hepatitis, } \\
\text { enteritis, common cold }\end{array}$ \\
\hline 281 & Raphanus sativus L. & Luobo萝卜 & $v \varepsilon^{3} \mid o \eta^{5} \mathrm{bu}^{3}$ & Cruciferae & Herb & Homegarden & Seed & $\begin{array}{l}\text { Grinding, decoction; Taken orally for senile } \\
\text { chronic bronchitis }\end{array}$ \\
\hline 282 & $\begin{array}{l}\text { Rauvolfia verticillata } \\
\text { (Lour.) Baill. }\end{array}$ & Luofumu萝芙木 & - & Apocynaceae & Shrub & Both & Root & $\begin{array}{l}\text { Pound fresh part applied on the affected area, } \\
\text { treating for bleeding, pain killer, hypertension, } \\
\text { dispersing blood stasis }\end{array}$ \\
\hline 283 & $\begin{array}{l}\text { Rehmannia glutinosa } \\
\text { (Gaertn.) Libosch. ex } \\
\text { Fisch. \& C.A. Mey. }\end{array}$ & Dihuang地黄 & $m^{6}{ }^{6} l i a o^{2} l i p^{7}$ & Scrophulariaceae & Herb & Wild & Tuber & $\begin{array}{l}\text { Grinding, decoction; Taken orally for removing } \\
\text { heat to promote salivation }\end{array}$ \\
\hline 284 & $\begin{array}{l}\text { Reineckia carnea (Andr.) } \\
\text { Kunth. }\end{array}$ & Jixiangcao吉祥草 & $\tan ^{6} k \partial p^{8}$ & Liliaceae & Herb & Wild & Whole plant & Grinding, decoction; Taken orally for bronchitis \\
\hline 285 & Reynoutria japonica Houtt. & Huzhang虎杖 & ruon$^{2}$ wan $^{6} \mathrm{chin}^{6}$ & Polygonaceae & Herb & Wild & Rhizome & $\begin{array}{l}\text { Grinding, decoction; Taken orally for cough, } \\
\text { blood stasis, rheumatism, traumatic injury, } \\
\text { jaundice, amenorrhea }\end{array}$ \\
\hline 286 & $\begin{array}{l}\text { Rhoeo discolor (L'Hér.) } \\
\text { Hance ex Walp. }\end{array}$ & $\begin{array}{l}\text { Zibeiwannianqing紫背 } \\
\text { 万年青 }\end{array}$ & ruon $^{2}$ phuon $^{2} w a^{3}$ & Commelinaceae & Herb & Wild & Flower & $\begin{array}{l}\text { Grinding, decoction; Taken orally for cough, } \\
\text { pertussis cough, diarrhea, hemoptysis, sore } \\
\text { throat, scrofula }\end{array}$ \\
\hline 287 & Rhus chinensis Mill. & Yanfumu盐麸木 & $m e^{4} w \omega t^{7}$ & Anacardiaceae & Shrub & Both & Cecidium & $\begin{array}{l}\text { Grinding, decoction; Taken orally for bleeding, } \\
\text { arrest sweating, piles, pharyngitis, inflammation }\end{array}$ \\
\hline 288 & Ricinus communis $\mathrm{L}$. & Bima菼麻 & thun ${ }^{6} \mathrm{ju}^{6}$ & Euphorbiaceae & Herb & Homegarden & Seed & $\begin{array}{l}\text { Pound fresh part applied on the affected area, } \\
\text { treating for scabies }\end{array}$ \\
\hline 289 & $\begin{array}{l}\text { Rorippa indica (L.) } \\
\text { Hiern }\end{array}$ & Hancai草菜 & $m a^{2} y o u^{5} y \varepsilon^{5}$ & Cruciferae & Herb & Wild & Whole plant & $\begin{array}{l}\text { Pound fresh part and mixed with rapeseed oil, } \\
\text { applied on the affected area, treating for } \\
\text { dermatitis }\end{array}$ \\
\hline 290 & Rosa chinensis Jacq. & Yuejihua月季花 & $\eta \varepsilon \eta^{4} \eta \varepsilon \eta^{4} Z \partial n^{5}$ & Rosaceae & Shrub & Homegarden & Flower & $\begin{array}{l}\text { Grinding, decoction; Taken orally for abnormal } \\
\text { menstruation }\end{array}$ \\
\hline 291 & Rosa laevigata Michx. & Jinyingzi金樱子 & $\operatorname{lak}^{8} \mathrm{man}^{4}$ & Rosaceae & Shrub & Both & Root, Fruit & $\begin{array}{l}\text { Grinding, decoction; Taken orally for bone } \\
\text { fracture, traumatic injury, appendicitis, diarrhea, } \\
\text { enteritis, stomachache }\end{array}$ \\
\hline 292 & Rosa multiflora Thunb. & Yeqiangwei野蔷薇 & - & Rosaceae & Shrub & Wild & Root, Seed & $\begin{array}{l}\text { Grinding, decoction; Taken orally for clearing } \\
\text { and activating the channels and collaterals, } \\
\text { diuresis }\end{array}$ \\
\hline 293 & Rosa roxburghii Tratt. & Saosihua缫丝花 & $\tan ^{5} \mathrm{~d} \varepsilon n m^{3} \operatorname{gan}^{4}$ & Rosaceae & Shrub & Wild & Root & $\begin{array}{l}\text { Grinding, decoction; Taken orally for indigestion, } \\
\text { stomachache }\end{array}$ \\
\hline 294 & Rubus parvifolius $\mathrm{L}$. & Maomei茅䒯 & $\operatorname{lak}^{8}$ thwm $^{6} \mathrm{pha}^{3}$ & Rosaceae & Herb & Wild & Whole plant & $\begin{array}{l}\text { Grinding the fresh part, decoction; Taken } \\
\text { orally for jaundice, toothache, chronic hepatitis, } \\
\text { stomachache, diarrhea, sphagitis }\end{array}$ \\
\hline 295 & Rumex nepalensis Spreng. & $\begin{array}{l}\text { Nibo'er'suanmo尼泊尔 } \\
\text { 酸模 }\end{array}$ & ma: ${ }^{6} 7 a n^{6} l o u^{5}$ & Polygonaceae & Herb & Wild & Root & Grinding, decoction; Taken orally for nephritis \\
\hline 296 & Salvia miltiorrhiza Bunge & Danshen丹参 & dan $\operatorname{sen}^{5}$ & Lamiaceae & Herb & Homegarden & Root & $\begin{array}{l}\text { Grinding, decoction; Taken orally for afterpains, } \\
\text { removing blood stasis }\end{array}$ \\
\hline
\end{tabular}


Table 1 Inventory of Medicinal Plants Traditionally Used by Maonan People (Continued)

\begin{tabular}{|c|c|c|c|c|c|c|c|c|}
\hline 297 & Sambucus chinensis Lindl. & Jiegucao接骨草 & - & Caprifoliaceae & Herb & Wild & Rhizome & $\begin{array}{l}\text { Grinding, decoction; Taken orally for rheumatic } \\
\text { arthritis, tonsillitis, rheumatoid arthritis, urinary } \\
\text { tract infection }\end{array}$ \\
\hline 298 & Sambucus williamsii Hance & Jiegumu接骨木 & ruon $^{2}$ raliem $^{2}$ & Caprifoliaceae & Shrub & Both & Leaf & $\begin{array}{l}\text { Grinding, decoction; Taken orally for traumatic } \\
\text { injury, rheumatic arthritis, waist and legs pain, } \\
\text { bone fracture, scapulohumeral periarthritis }\end{array}$ \\
\hline 299 & Sanguisorba officinalis L. & Diyu地榆 & $g a n^{5} g u^{2} v a^{3}$ & Rosaceae & Herb & Wild & Root & $\begin{array}{l}\text { Grinding the fresh part, decoction; Taken orally } \\
\text { for diarrhea }\end{array}$ \\
\hline 300 & Sapindus mukorossi Gaertn. & Wuhuanzi无患子 & ruon $^{2} l a k^{8}$ rək $^{7}$ & Sapindaceae & Tree & Wild & Seed & $\begin{array}{l}\text { Grinding, decoction; Taken orally for tuberculosis, } \\
\text { pertussis cough }\end{array}$ \\
\hline 301 & $\begin{array}{l}\text { Sapium discolor } \\
\text { (Champ. ex Benth.) Müll. Arg. }\end{array}$ & Shanwujiu山乌柏 & ruon ${ }^{2} m e i^{4} \partial k^{7}$ & Euphorbiaceae & Tree & Both & Leaf & $\begin{array}{l}\text { Grinding, decoction; Taken orally for traumatic } \\
\text { injury, snake bite, constipation, carbuncle }\end{array}$ \\
\hline 302 & $\begin{array}{l}\text { Sargentodoxa cuneata } \\
\text { (Oliv.) Rehder \& E.H. Wilson }\end{array}$ & Daxueteng大血藤 & bieu $^{3}$ phiat $^{7}$ & Lardizabalaceae & Liana & Wild & Root, Stem & $\begin{array}{l}\text { Medicinal liquor for treating rheumatic arthritis, } \\
\text { traumatic injury, ostealgia }\end{array}$ \\
\hline 303 & Saurauia tristyla DC. & Shuidongge水东哥 & - & Actinidiaceae & Shrub & Homegarden & Root & $\begin{array}{l}\text { Grinding, decoction; Taken orally for carbuncle, } \\
\text { cough, bronchitis, toothache }\end{array}$ \\
\hline 304 & Saururus chinensis (Lour.) Baill. & Sanbaicao三白草 & ruon $^{2} s \omega t^{7} \mathrm{mbei}^{1}$ & Saururaceae & Herb & Both & Whole plant & $\begin{array}{l}\text { Grinding, decoction; Taken orally for nephritis, } \\
\text { edema, lithangiuria, eczema, furuncle, carbuncle }\end{array}$ \\
\hline 305 & Saxifraga stolonifera Curtis & Hu'er'cao虎耳草 & ruon ${ }^{2} \mathrm{kha}^{3} \mathrm{~m} \omega \mathrm{m}^{4}$ & Saxifragaceae & Herb & Wild & Leaf & $\begin{array}{l}\text { Pound fresh part applied on the affected area, } \\
\text { treating for traumatic hemorrhage, furuncle, } \\
\text { parotitis, empyrosis }\end{array}$ \\
\hline 306 & $\begin{array}{l}\text { Schefflera heptaphylla (L.) } \\
\text { Frodin }\end{array}$ & E'zhangchai鹅掌柴 & $m e i^{5} \operatorname{dian}^{2} 7 \varepsilon p^{8}$ & Araliaceae & Tree & Both & $\begin{array}{l}\text { Root Bark, Stem } \\
\text { Bark, Leaf }\end{array}$ & $\begin{array}{l}\text { Grinding and decoction; Taken orally; Root } \\
\text { and Stem bark are for fever, rheumatism, } \\
\text { ostealgia, traumatic injury, sore throat; Leaf } \\
\text { is for eczema, allergic dermatitis }\end{array}$ \\
\hline 307 & $\begin{array}{l}\text { Schizocapsa plantaginea } \\
\text { Hance }\end{array}$ & Lieguoshu裂果薯 & suei $i^{1}{ }^{6} \mathrm{pu}^{4}$ & Taccaceae & Herb & Wild & Rhizome & $\begin{array}{l}\text { Grinding, decoction; Taken orally for cough, } \\
\text { traumatic injury, pharyngitis, heart and } \\
\text { stomach pain }\end{array}$ \\
\hline 308 & Scutellaria barbata D. Don & Banzhilian半枝莲 & ruon $^{2} w o k^{7} \lim ^{6} s \varepsilon^{5}$ & Lamiaceae & Herb & Wild & Whole plant & $\begin{array}{l}\text { Grinding, decoction; Taken orally for cancer, } \\
\text { appendicitis, hepatitis and hepatic ascites }\end{array}$ \\
\hline 309 & $\begin{array}{l}\text { Selaginella moellendorffii } \\
\text { Hieron. }\end{array}$ & $\begin{array}{l}\text { Jiangnanjuanbai江南卷 } \\
\text { 柏 }\end{array}$ & ?guit ${ }^{7} \varepsilon^{2}$ bua $^{5}$ & Selaginellaceae & - & Wild & Whole plant & $\begin{array}{l}\text { Pound fresh part applied on the affected } \\
\text { area, treating for hematoma after contusion }\end{array}$ \\
\hline 310 & $\begin{array}{l}\text { Selaginella tamariscina } \\
\text { (P. Beauv.) Spring }\end{array}$ & Juanbai卷柏 & ruon ${ }^{2}$ sai thui ${ }^{2}$ & Selaginellaceae & - & Wild & Whole plant & $\begin{array}{l}\text { Grinding, decoction; Taken orally for hemafecia, } \\
\text { epistaxis, metrorrhagia, traumatic injury, chronic } \\
\text { hepatitis, proctoptosis }\end{array}$ \\
\hline 311 & $\begin{array}{l}\text { Semiaquilegia adoxoides } \\
\text { (DC.) Makino }\end{array}$ & Tiankui天葵 & $m a^{3} \gamma e^{5} n o^{2}$ & Ranunculaceae & Herb & Wild & Tuber & $\begin{array}{l}\text { Grinding, decoction; Taken orally for } \\
\text { stomachache }\end{array}$ \\
\hline 312 & $\begin{array}{l}\text { Senecio scandens } \\
\text { Buch.-Ham. ex D. Don }\end{array}$ & Qianliguang千里光 & $w a^{3} n u k^{8} s^{5}$ & Asteraceae & Herb & Wild & Whole plant & $\begin{array}{l}\text { Grinding, decoction; Taken orally for fever, } \\
\text { jaundiced hepatitis, throat ache, mumps, } \\
\text { bleeding, eczema }\end{array}$ \\
\hline 313 & Senna occidentalis (L.) Link & Wangjiangnan望江南 & - & Fabaceae & Shrub & Both & Seed & $\begin{array}{l}\text { Grinding, decoction; Taken orally for habitual } \\
\text { constipation, hypertension, headache, } \\
\text { indigestion, epifolliculitis, oral mucosa ulcer }\end{array}$ \\
\hline
\end{tabular}


Table 1 Inventory of Medicinal Plants Traditionally Used by Maonan People (Continued)

\begin{tabular}{|c|c|c|c|c|c|c|c|c|}
\hline 314 & $\begin{array}{l}\text { Serissa japonica (Thunb.) } \\
\text { Thunb. }\end{array}$ & Liuyuexue六月雪 & $\tan ^{5}$ ?nui ${ }^{5}$ wai $^{3}$ & Rubiaceae & Shrub & Wild & Whole plant & $\begin{array}{l}\text { Grinding, decoction; Taken orally for infantile } \\
\text { convulsions }\end{array}$ \\
\hline 315 & Setcreasea purpurea Boom & Zizhumei紫竹梅 & - & Commelinaceae & Herb & Wild & Whole plant & $\begin{array}{l}\text { Pound fresh part applied on the affected area, } \\
\text { treating for bleeding, snake bite, activating } \\
\text { blood and herpes }\end{array}$ \\
\hline 316 & Sida szechuensis Matsuda & Badusan拔毒散 & - & Malvaceae & Shrub & Wild & Whole plant & $\begin{array}{l}\text { Pound fresh part applied on the affected area, } \\
\text { treating for traumatic injury and inflammation }\end{array}$ \\
\hline 317 & Sigesbeckia orientalis L. & Xixian狶莶 & wok ${ }^{7}$ cut $^{7}$ btio $^{1}$ & Asteraceae & Herb & Wild & Whole plant & $\begin{array}{l}\text { Grinding, decoction; Taken orally for insomnia, } \\
\text { hypertension, acute jaundiced hepatitis, } \\
\text { diarrhea, malaria, numbness of limbs }\end{array}$ \\
\hline 318 & Smilax glabra Roxb. & Tufuling土获苓 & $\mid a k^{8} \mathrm{~d} \mathrm{~m}^{4} \mathrm{sei}^{1}$ & Smilacaceae & Herb & Homegarden & Rhizome & $\begin{array}{l}\text { Grinding, decoction; Taken orally for nephritis, } \\
\text { diarrhea, detoxication, arthralgia }\end{array}$ \\
\hline 319 & Solanum capsicoides All. & Niuqiezi牛茄子 & - & Solanaceae & Herb & Wild & Whole plant & $\begin{array}{l}\text { Grinding, decoction; Taken orally for fever } \\
\text { due to common cold, headache, cough, } \\
\text { abscess, chest stuffiness }\end{array}$ \\
\hline 320 & Solanum violaceum $\mathrm{L}$. & Citianqie刺天茄 & lak $^{8} \mathrm{khat}^{8} \mathrm{se}^{5}$ & Solanaceae & Shrub & Wild & Leaf, Fruit & $\begin{array}{l}\text { Pound fresh part applied on the affected area, } \\
\text { treating for yellow-water ulcer, fingers ulcer } \\
\text { and ringworm }\end{array}$ \\
\hline 321 & Solidago decurrens Lour. & $\begin{array}{l}\text { Yizhihuanghua一枝黄 } \\
\text { 花 }\end{array}$ & wok $k^{7} a^{3} \operatorname{man}^{1}$ & Asteraceae & Herb & Wild & Whole plant & $\begin{array}{l}\text { Grinding, decoction; Taken orally for fever, } \\
\text { headache, jaundice, bronchitis, acute gastricism, } \\
\text { upper respiratory infection, swelling, throat ache }\end{array}$ \\
\hline 322 & Sophora flavescens Aiton & Kushen苦参 & ruon $^{2} \operatorname{yau}^{3} \mathrm{in}^{5}$ & Fabaceae & Herb & Wild & Rhizome & $\begin{array}{l}\text { Grinding, decoction; Taken orally for piles, } \\
\text { cutaneous pruritus }\end{array}$ \\
\hline 323 & Sophora tonkinensis Gagnep. & Yuenanhuai越南槐 & - & Fabaceae & Shrub & Wild & Root & $\begin{array}{l}\text { Grinding, decoction; Taken orally for acute } \\
\text { pharyngitis, tonsillitis, swelling and aching } \\
\text { of gum, cough, constipation }\end{array}$ \\
\hline 324 & $\begin{array}{l}\text { Sparganium stoloniferum } \\
\text { (Buch.-Ham. ex Graebn.) } \\
\text { Buch.-Ham. ex Juz. }\end{array}$ & Heisanleng黑三棱 & - & Sparganiaceae & Herb & Wild & Tuber & $\begin{array}{l}\text { Grinding, decoction; Taken orally for blood } \\
\text { stasis, pain killer }\end{array}$ \\
\hline 325 & $\begin{array}{l}\text { Spatholobus sinensis } \\
\text { Chun \& T.C. Chen }\end{array}$ & Hongxueteng红血藤 & ruon $^{2}$ pu'phiat $^{7}$ & Fabaceae & Liana & Wild & Stem & Medicinal liquor for treating traumatic injury \\
\hline 326 & Spatholobus suberectus Dunn & Mihuadou密花豆 & - & Fabaceae & Liana & Wild & Stem & $\begin{array}{l}\text { Grinding, decoction; Taken orally for } \\
\text { stomachache, enriching blood, waist and } \\
\text { knees pain }\end{array}$ \\
\hline 327 & $\begin{array}{l}\text { Spiranthes sinensis (Pers.) } \\
\text { Ames }\end{array}$ & Shoucao绶草 & ruon $^{2}$ thou $^{6}$ nen ${ }^{4}$ & Orchidaceae & Herb & Wild & Whole plant, Root & $\begin{array}{l}\text { Grinding, decoction; Taken orally for diabetes, } \\
\text { leucorrhoea, weakness, sore throat, } \\
\text { neurasthenia and erectile dysfunction }\end{array}$ \\
\hline 328 & $\begin{array}{l}\text { Stahlianthus involucratus } \\
\text { (King ex Baker) Craib }\end{array}$ & Tutianqi土田七 & ruon $^{2}$ in ${ }^{3}$ don ${ }^{2}$ & Zingiberaceae & Herb & Both & Tuber & $\begin{array}{l}\text { Grinding, decoction; Taken orally for } \\
\text { traumatic injury, rheumatism, ostealgia }\end{array}$ \\
\hline 329 & Stemona tuberosa Lour. & Dabaibu大百部 & $\operatorname{lak}^{8} \mathrm{ru}^{3} \mathrm{khui}{ }^{2}$ & Stemonaceae & Liana & Wild & Tuber & $\begin{array}{l}\text { Grinding, decoction; Taken orally for pertussis } \\
\text { cough, tuberculosis, bronchitis }\end{array}$ \\
\hline 330 & $\begin{array}{l}\text { Stephania cepharantha } \\
\text { Hayata }\end{array}$ & $\begin{array}{l}\text { Jinxiandiaowugui金线 } \\
\text { 吊乌龟 }\end{array}$ & $m \varepsilon i^{5} \mathrm{miu}^{2}$ & Menispermaceae & Liana & Wild & Tuber & $\begin{array}{l}\text { Pound fresh part applied on the affected } \\
\text { area, treating for carbuncle, snake bite }\end{array}$ \\
\hline
\end{tabular}


Table 1 Inventory of Medicinal Plants Traditionally Used by Maonan People (Continued)

\begin{tabular}{|c|c|c|c|c|c|c|c|c|}
\hline 331 & $\begin{array}{l}\text { Streptocaulon juventas (Lour.) } \\
\text { Merr. }\end{array}$ & Anxiaoteng暗消藤 & - & Asclepiadaceae & Liana & Wild & Root, Leaf & $\begin{array}{l}\text { Grinding, decoction; Taken orally; Root is for } \\
\text { diarrhea, piles, pneumonia, vitiligo and } \\
\text { arrhythmia; Pound fresh leaf applied on } \\
\text { the affected area, treating for snake bite, } \\
\text { eczema and vaginitis }\end{array}$ \\
\hline 332 & Striga asiatica (L.) Kuntze & Dujiaojin 独脚金 & ruon $^{2} \mathrm{ra}^{2} \mathrm{mei}^{3}$ & Scrophulariaceae & Herb & Wild & Whole plant & $\begin{array}{l}\text { Grinding, decoction; Taken orally for infantile } \\
\text { malnutrition, dampness-heat constitution, } \\
\text { diarrhea, jaundiced hepatitis }\end{array}$ \\
\hline 333 & $\begin{array}{l}\text { Strophanthus divaricatus } \\
\text { (Lour.) Hook. \& Arn. }\end{array}$ & Yangjiaoniu羊角拗 & - & Apocynaceae & Shrub & Wild & Stem, Leaf & $\begin{array}{l}\text { Grinding, decoction; Taken orally for rheumatic } \\
\text { arthritis, traumatic injury, snake bite, sprain }\end{array}$ \\
\hline 334 & $\begin{array}{l}\text { Tadehagi triquetrum } \\
\text { (L.) H. Ohashi }\end{array}$ & Hulucha葫芦茶 & tsha ${ }^{2} j a^{1}$ & Fabaceae & Shrub & Both & Whole plant & $\begin{array}{l}\text { Grinding, decoction; Taken orally for nephritis, } \\
\text { enteritis, diarrhea, hepatitis }\end{array}$ \\
\hline 335 & $\begin{array}{l}\text { Talinum paniculatum } \\
\text { (Jacq.) Gaertn. }\end{array}$ & Turenshen土人参 & $\mathrm{kau}^{5} \mathrm{Ii}^{6} s \omega n^{1}$ & Portulacaceae & Herb & Wild & Root & $\begin{array}{l}\text { Boiled with meat and drunk the soup, treating } \\
\text { for moistening lung, health tonic }\end{array}$ \\
\hline 336 & $\begin{array}{l}\text { Taraxacum mongolicum } \\
\text { Hand.-Mazz. }\end{array}$ & Pugongying蒲公英 & $\mathrm{mba}^{3} \mathrm{~kat}^{7} \mathrm{sei}^{1}$ & Asteraceae & Herb & Wild & Whole plant & $\begin{array}{l}\text { Grinding, decoction; Taken orally for } \\
\text { conjunctivitis, epidemic parotitis, enteritis, } \\
\text { gastricism, hepatitis, diarrhea, acute mastitis, } \\
\text { sphagitis }\end{array}$ \\
\hline 337 & Taxillus chinensis (DC.) Danser & Guangjisheng广寄生 & - & Loranthaceae & Shrub & Wild & Whole plant & $\begin{array}{l}\text { Grinding, decoction; Taken orally for numbness } \\
\text { of limbs, rheumatism, ostealgia, arthritis, lumbar } \\
\text { muscle degeneration }\end{array}$ \\
\hline 338 & $\begin{array}{l}\text { Tetrapanax papyrifer } \\
\text { (Hook.) K. Koch }\end{array}$ & Tongtuomu通脱木 & tai $^{5}$ pon $^{2}$ & Araliaceae & Shrub & Homegarden & Stem pith & $\begin{array}{l}\text { Boiled with meat and drunk the soup, treating } \\
\text { for promoting lactation }\end{array}$ \\
\hline 339 & $\begin{array}{l}\text { Tetrastigma planicaule } \\
\text { (Hook. f.) Gagnep. }\end{array}$ & Biandanteng扁担藤 & m7au $\mathrm{bi \varepsilon n}^{2}$ & Vitaceae & Liana & Both & Root, Stem & Fried the root or stem, fumigation for pinkeye \\
\hline 340 & $\begin{array}{l}\text { Tinospora sagittata (Oliv.) } \\
\text { Gagnep. }\end{array}$ & Qingniudan青牛胆 & $k \supset \eta^{1} p i \varepsilon \eta^{5} v i^{3}$ & Menispermaceae & Liana & Wild & Tuber & $\begin{array}{l}\text { Powder, dispersed in water and drunk for acute } \\
\text { gastroenteritis, acute pharyngitis, bacillary } \\
\text { diarrhea, appendicitis }\end{array}$ \\
\hline 341 & $\begin{array}{l}\text { Tinospora sinensis (Lour.) } \\
\text { Merr. }\end{array}$ & $\begin{array}{l}\text { Zhonghuaqingniudan中 } \\
\text { 华青牛胆 }\end{array}$ & yuon$^{2}$ son $^{3} \mathrm{jin}^{3}$ & Menispermaceae & Liana & Wild & Stem & $\begin{array}{l}\text { Grinding, decoction; Taken orally for } \\
\text { rheumatism, traumatic injury, lumbar } \\
\text { muscle degeneration, sciatica }\end{array}$ \\
\hline 342 & Toddalia asiatica (L.) Lam. & $\begin{array}{l}\text { Feilongzhangxue 飞龙 } \\
\text { 掌血. }\end{array}$ & $\operatorname{cim}^{3} \mathrm{ce}^{3} \operatorname{vin}^{1}$ & Rutaceae & Liana & Wild & Root Bark & $\begin{array}{l}\text { Pound fresh part applied on the affected area, } \\
\text { treating for traumatic injury, skin disease, } \\
\text { relieving pain, detumescence }\end{array}$ \\
\hline 343 & $\begin{array}{l}\text { Trachelospermum jasminoides } \\
\text { (Lindl.) Lem. }\end{array}$ & Luoshi络石 & - & Apocynaceae & Liana & Wild & Whole plant & $\begin{array}{l}\text { Pound fresh part applied on the affected area, } \\
\text { treating for bleeding, rheumatism, waist pain, } \\
\text { dispersing blood stasis }\end{array}$ \\
\hline 344 & $\begin{array}{l}\text { Trachycarpus fortunei } \\
\text { (Hook.) H. Wendl. }\end{array}$ & Zonglü区榈 & $w e i^{5}$ & Palmae & Tree & Homegarden & Leaf, Fruit & $\begin{array}{l}\text { Boiled with meat and drunk the soup, treating } \\
\text { for epilepsy }\end{array}$ \\
\hline 345 & Trichosanthes kirilowii Maxim. & Gualou栝楼 & - & Cucurbitaceae & Liana & Wild & Root & $\begin{array}{l}\text { Grinding, decoction; Taken orally for removing } \\
\text { heat to promote salivation, expel pus and } \\
\text { disperse swelling }\end{array}$ \\
\hline
\end{tabular}


Table 1 Inventory of Medicinal Plants Traditionally Used by Maonan People (Continued)

\begin{tabular}{|c|c|c|c|c|c|c|c|c|}
\hline 346 & Trichosanthes rosthornii Harms & $\begin{array}{l}\text { Zhonghuagualou中华 } \\
\text { 栝楼 }\end{array}$ & gua ${ }^{5} e^{5} m a^{2}$ & Cucurbitaceae & Liana & Wild & Shuck, Seed & Grinding, decoction; Taken orally for edema \\
\hline 347 & $\begin{array}{l}\text { Typhonium blumei Nicolson \& } \\
\text { Sivad. }\end{array}$ & Litoujian犁头尖 & $\operatorname{lak}^{8} \mathrm{chieu}^{4} \mathrm{~d} \partial \eta^{2}$ & Araceae & Herb & Wild & Tuber & $\begin{array}{l}\text { Pound fresh part applied on the affected area, } \\
\text { treating for snake bite, scrofula, traumatic injury, } \\
\text { hemangioma and furuncle }\end{array}$ \\
\hline 348 & Typhonium giganteum Engl. & Dujiaolian独角莲 & - & Araceae & Herb & Both & Tuber & $\begin{array}{l}\text { Grinding, decoction; Taken orally for } \\
\text { gastroduodenal ulcer }\end{array}$ \\
\hline 349 & $\begin{array}{l}\text { Uncaria rhynchophylla } \\
\text { (Miq.) Miq. ex Havil. }\end{array}$ & Gouteng钩藤 & $m e i^{5} g ? a u^{2} d a u^{3}$ & Rubiaceae & Liana & Wild & Hooked stem & $\begin{array}{l}\text { Grinding, decoction; Taken orally for jaundiced } \\
\text { hepatitis, dizziness, headach, calming the liver }\end{array}$ \\
\hline 350 & Urena lobata L. & Ditaohua地桃花 & ruon $^{2}$ wok $^{7} \mathrm{cut}^{7}$ & Malvaceae & Herb & Wild & Whole plant & $\begin{array}{l}\text { Grinding, decoction; Taken orally for fever, } \\
\text { diarrhea, enteritis, malaria; Pound fresh part } \\
\text { applied on the affected area, treating for } \\
\text { traumatic injury, bone fracture, snake bite, } \\
\text { mastitis }\end{array}$ \\
\hline 351 & Valeriana jatamansi Jones & Zhizhuxiang蜘蛛香 & $m a^{2} v a^{3}$ & Valerianaceae & Herb & Wild & Rhizome & $\begin{array}{l}\text { Pound fresh part applied on the affected } \\
\text { area, treating for furuncle }\end{array}$ \\
\hline 352 & Ventilago leiocarpa Benth. & Yiheguo翼核果 & - & Rhamnaceae & Shrub & Wild & Root & $\begin{array}{l}\text { Pound fresh part applied on the affected } \\
\text { area, treating for traumatic injury, rheumatism, } \\
\text { numbness of limbs, edema and menorrhagia }\end{array}$ \\
\hline 353 & Verbena officinalis $\mathrm{L}$. & Mabiancao马鞭草 & ruon $^{2}$ pien $^{3}$ mia $^{4}$ & Verbenaceae & Herb & Wild & Whole plant & $\begin{array}{l}\text { Grinding, decoction; Taken orally for hypertension, } \\
\text { diarrhea, malaria, nephritis, fever due to common } \\
\text { cold, urinary tract infection }\end{array}$ \\
\hline 354 & Vernonia cinerea (L.) Less. & Yexiangniu夜香牛 & ruon $\left.^{2} \mathrm{~m} \mathrm{~m}^{5} \mathrm{ndan}\right)^{3}$ & Asteraceae & Herb & Wild & Whole plant & $\begin{array}{l}\text { Pound fresh part applied on the affected } \\
\text { area, treating for snake bite, swelling, furuncle }\end{array}$ \\
\hline 355 & Viola inconspicua Blume & Chang'e'jincai长蘦堇菜 & $v a^{5} \mathrm{mba}^{3} k u e i^{3}$ & Violaceae & Herb & Wild & Whole plant & $\begin{array}{l}\text { Grinding, decoction; Taken orally for } \\
\text { pharyngitis, jaundice, diarrhea, swelling, } \\
\text { pain of eye }\end{array}$ \\
\hline 356 & Viola philippica Cav. & Zihuadiding紫花地丁 & $y a^{5} m \varepsilon p^{8} \mid i^{2}$ & Violaceae & Herb & Wild & Whole plant & $\begin{array}{l}\text { Grinding, decoction; Taken orally for } \\
\text { appendicitis, piles }\end{array}$ \\
\hline 357 & $\begin{array}{l}\text { Viscum liquidambaricolum } \\
\text { Hayata }\end{array}$ & $\begin{array}{l}\text { Fengxianghujisheng枫 } \\
\text { 香葪寄生 }\end{array}$ & $\operatorname{sap}^{7} m e^{4} h u^{3}$ & Loranthaceae & Shrub & Wild & Whole plant & $\begin{array}{l}\text { Grinding, decoction; Taken orally for lumbar } \\
\text { muscle degeneration, cough, traumatic injury, } \\
\text { rheumatic arthritis }\end{array}$ \\
\hline 358 & Vitex negundo L. & Huangjing黄荆 & $m e i^{4} \mathrm{cin}^{3}$ & Verbenaceae & Shrub & Wild & Stem, Leaf & $\begin{array}{l}\text { Grinding, decoction; Taken orally for diarrhea, } \\
\text { malaria, enteritis, common cold, heatstroke }\end{array}$ \\
\hline 359 & Vitex trifolia L. & Manjing蔓荆 & $m e^{5} t_{b i \varepsilon^{2}} z a^{2}$ & Verbenaceae & Shrub & Wild & Fruit & Powder, swallowed for headache \\
\hline 360 & $\begin{array}{l}\text { Wikstroemia indica (L.) } \\
\text { C.A. Mey. }\end{array}$ & Liaogewang了哥王 & ruon $^{2}|j \varepsilon n| j e u^{4}$ & Thymelaeaceae & Shrub & Wild & Stem, Leaf & $\begin{array}{l}\text { Grinding, decoction; Taken orally for clearing } \\
\text { away heat and toxic materials, traumatic injury, } \\
\text { hepatitis, parotitis }\end{array}$ \\
\hline 361 & $\begin{array}{l}\text { Woodwardia japonica } \\
\text { (L. f.) Sm. }\end{array}$ & Gouji狗脊 & $\operatorname{wan}^{6} \mathrm{cin}^{5} \mathrm{kou}^{1}$ & Blechnaceae & Herb & Wild & Rhizome & $\begin{array}{l}\text { Grinding, decoction; Taken orally for } \\
\text { neurasthenia, rheumatic arthralgia, diuresis, } \\
\text { waist and knees pain }\end{array}$ \\
\hline
\end{tabular}


Table 1 Inventory of Medicinal Plants Traditionally Used by Maonan People (Continued)

\begin{tabular}{|c|c|c|c|c|c|c|c|c|}
\hline 362 & Wrightia laevis Hook. f. & Lanshu蓝树 & - & Apocynaceae & Tree & Homegarden & Root, Leaf & $\begin{array}{l}\text { Grinding, decoction; Taken orally for bleeding, } \\
\text { traumatic injury, mumps }\end{array}$ \\
\hline 363 & $\begin{array}{l}\text { Xanthium sibiricum Patrin ex } \\
\text { Widder }\end{array}$ & Cang'er苍耳 & wok $^{7}$ cut $^{7} l a u^{4}$ & Asteraceae & Herb & Wild & Fruit & $\begin{array}{l}\text { Pound after fried and drunk with yellow wine } \\
\text { for enteritis, rheumatic arthralgia, headache }\end{array}$ \\
\hline 364 & Zanthoxylum armatum DC. & Zhuyehuajiao竹叶花椒 & $\operatorname{lak}^{8} x i e u^{3} n a^{4}$ & Rutaceae & Tree & Both & Fruit & $\begin{array}{l}\text { Grinding, decoction; Taken orally for traumatic } \\
\text { injury, chronic gastricism, cough, depriving } \\
\text { ascarid }\end{array}$ \\
\hline 365 & $\begin{array}{l}\text { Zanthoxylum nitidum } \\
\text { (Roxb.) DC. }\end{array}$ & Liangmianzhen两面针 & $\operatorname{lak}^{8} \mathrm{xieu}^{3} \mathrm{don} \eta^{2}$ & Rutaceae & Liana & Wild & Root, Stem, Leaf & $\begin{array}{l}\text { Grinding, decoction; Taken orally for duodena } \\
\text { ulcer, traumatic injury, rheumatism, diarrhea, } \\
\text { malaria, chronic gastricism }\end{array}$ \\
\hline 366 & Zea mays $\mathrm{L}$. & Yumi玉米 & nui ${ }^{5} w^{5} i^{5} \mathrm{die}^{3}$ & Gramineae & Herb & Homegarden & Column & Powder swallowed for diabetes \\
\hline 367 & $\begin{array}{l}\text { Zehneria indica (Lour.) } \\
\text { Keraudren }\end{array}$ & $\begin{array}{l}\text { Laoshuladonggua老鼠 } \\
\text { 拉冬瓜 }\end{array}$ & $\operatorname{lak}^{8} \mathrm{kua}^{3} \mathrm{no}^{1}$ & Cucurbitaceae & Liana & Wild & Whole plant & $\begin{array}{l}\text { Grinding, decoction; Taken orally for urinary } \\
\text { tract infection, tonsillitis, acute conjunctivitis, } \\
\text { carbuncle }\end{array}$ \\
\hline 368 & Ziziphus jujuba Mill. & Zao杳 & $z a: o^{3} z i:^{2}$ & Rhamnaceae & Tree & Homegarden & Fruit & $\begin{array}{l}\text { Grinding, decoction; Taken orally for infantile } \\
\text { diarrhea }\end{array}$ \\
\hline
\end{tabular}


Table 2 Taxonomic diversity of medicinal plants in the study area

\begin{tabular}{lllll}
\hline Family & $\begin{array}{l}\text { Number } \\
\text { of genera }\end{array}$ & $\begin{array}{l}\text { Percentage } \\
\text { (\%) }\end{array}$ & $\begin{array}{l}\text { Number } \\
\text { of species }\end{array}$ & $\begin{array}{l}\text { Percentage of } \\
\text { species (\%) }\end{array}$ \\
\hline Asteraceae & 22 & 7.46 & 24 & 6.52 \\
Fabaceae & 15 & 5.08 & 19 & 5.16 \\
Rosaceae & 11 & 3.73 & 16 & 4.35 \\
Euphorbiaceae & 8 & 2.71 & 14 & 3.80 \\
Liliaceae & 9 & 3.05 & 13 & 3.53 \\
Araceae & 7 & 2.37 & 11 & 2.99 \\
Lamiaceae & 9 & 3.05 & 9 & 2.45 \\
Polygonaceae & 4 & 1.36 & 8 & 2.17 \\
Zingiberaceae & 4 & 1.36 & 8 & 2.17 \\
Lauraceae & 4 & 1.36 & 7 & 1.90 \\
Ranunculaceae & 6 & 2.03 & 7 & 1.90 \\
Rutaceae & 5 & 1.69 & 7 & 1.90 \\
Asclepiadaceae & 4 & 1.36 & 7 & 1.90 \\
Cucurbitaceae & 6 & 2.03 & 7 & 1.90 \\
Gramineae & 6 & 2.03 & 6 & 1.63 \\
Araliaceae & 4 & 1.36 & 6 & 1.63 \\
Rubiaceae & 6 & 2.03 & 6 & 1.63 \\
Verbenaceae & 4 & 1.36 & 6 & 1.63 \\
Other families & 162 & 54.92 & 188 & 51.09 \\
Total & 295 & 100 & 368 & 100 \\
\hline & & & &
\end{tabular}

while some exotic or difficult-accessed species were bought from medicinal materials suppliers. Generally fresh parts were wild harvest. Most medicinal plants were not available from local market, only some species were found to be sold but mainly for their uses as spice or food, such as Zanthoxylum armatum, Nepeta cataria and Houttuynia cordata.

Table 3 Demographic profile of informants

\begin{tabular}{lll}
\hline Indicator & Description & Frequency (\%) \\
\hline Age & $20-29$ & $7(5.9)$ \\
& $30-39$ & $23(19.5)$ \\
& $40-49$ & $38(32.2)$ \\
& $50-59$ & $29(24.6)$ \\
& $60-69$ & $12(10.2)$ \\
& $70-79$ & $5(4.2)$ \\
Gender & $\geq 80$ & $4(3.4)$ \\
Education & Male & $106(89.8)$ \\
& Female & $12(10.2)$ \\
& None & $27(22.9)$ \\
& Primary & $72(61.0)$ \\
& Secondary & $13(11.0)$ \\
& Tertiary & $6(5.1)$ \\
\hline
\end{tabular}

\section{Diseases treated in the study area}

The medicinal plants were used to treat 95 human ailments in the study area. With regard to human diseases, traumatic injury was the one against which a high number of medicinal plants (67 species) were prescribed, followed by diarrhea (65 species), cough (44 species), hepatitis (37 species), enteritis (35 species), rheumatism (30 species), arthritis (27 species), bleeding (26 species), snake bite (24 species), furuncle (22 species) and nephritis (22 species).

The highest number of species $(139,37.57 \%)$ was used for the treatment of internal organs like liver (hepatitis, cirrhosis, jaundice, hepatic ascites, hepatosplenomegaly and so on), stomach (stomachache, stomach ulcer, gastroduodenal ulcer, flatulence, gastricism, indigestion and poor appetite), enteron (enteritis, proctoptosis, appendicitis and so on), spleen and diarrhea, with 251 (20.69\%) of all conditions (Table 4 Rheumatic problems (83 species used, 22.4\%) were mentioned as 140 (11.54\%) of all uses; 83 species $(22.4 \%)$ were used to treat respiratory problems, with 112 applications (9.23\%). Bone problems were treated with 72 species $(19.46 \%)$, with 85 conditions $(7.01 \%)$. Skin problems were mentioned in 87 uses (7.17\%), with 65 species (17.57\%) used for treatment. Inflammation was treated with 48 species (12.97\%), and comprised 67 (5.52\%) of all conditions (Table 4).

Ranking, informant consensus factor and multipurpose of medicinal plants

Among all ailments in the villages surveyed, traumatic injury was the most commonly disease against which a high number of medicinal plants (67 species) were prescribed. Seven medicinal plant species were used effectively for treating traumatic injury according to key informants. The results revealed Gaultheria leucocarpa was the most preferred medicinal plant, followed by Acanthopanax trifoliatus, and Sargentodoxa cuneata (Table 5).

Table 4 gave an overview of the main illness categories. The diseases that were prevalent in the study area had relatively higher $\mathrm{F}_{\mathrm{IC}}$ values. Medicinal plants to treat certain disease effectively and with reputation in Maonan communities also have higher $\mathrm{F}_{\mathrm{IC}}$ : traumatic injury and sprain (0.74), fever and malaria (0.73) and infantile diseases (0.75). Moreover, informants indicated the effectiveness of traditional medicines to get relief from certain diseases including traumatic injury, bone fracture, health problems associated with the liver disorder, snake bite, and spider poisoning.

The Maonans naturally relied on plants for multipurpose. Table 6 showed the most frequently inventoried medicinal plants had more functions used by the Maonans in local societies. In addition to medicinal value, most of medicinal plants were also valued for their economic, edible and ornamental values which were considered to serve an 


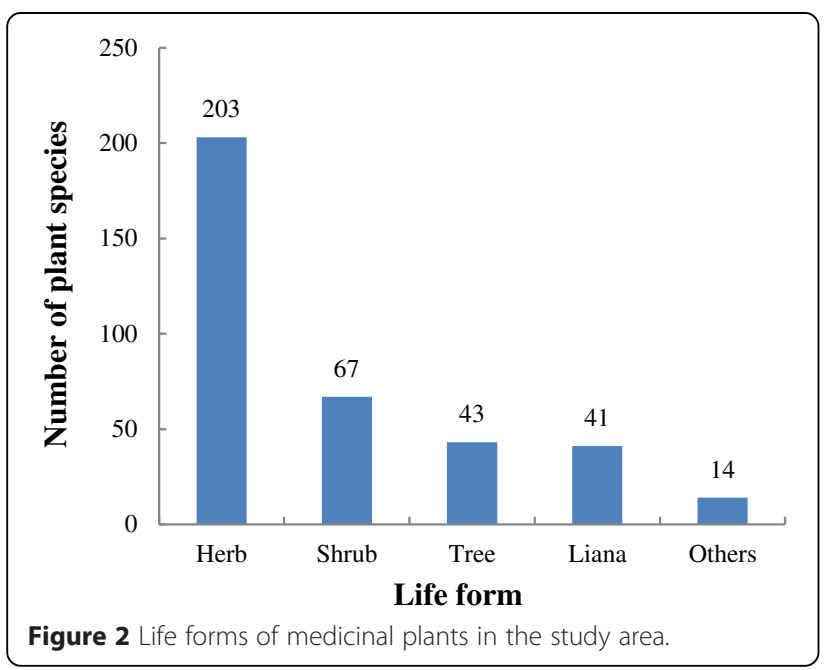

ecological role in the study sites. These plants included Acanthopanax trifoliatus, Litsea pungens, Platycodon grandiflorus, Rubus parvifolius, and Talinum paniculatum. Besides their medicinal purpose, these plants were sold in the local markets for the purposes of foods, spices and herbal teas, such as Allium fistulosum, Allium tuberosum, Cinnamomum cassia, Perilla frutescens, Oenanthe javanica, Gardenia jasminoides, Houttuynia cordata, and Juglans regia.

\section{Mode of preparation, condition, dosage of application}

Various plant species were collected and used immediately. Most of the medicinal formulations were administrated orally in ailment categories other than dermatological problems. In dermatological ailments, plants were administrated externally. Water and some additives were often used in the preparation of remedies, such as alcohol, oil, honey, salt, sugar, eggs, chicken, duck and meat. The additives were claimed to either increase nutrition or improve

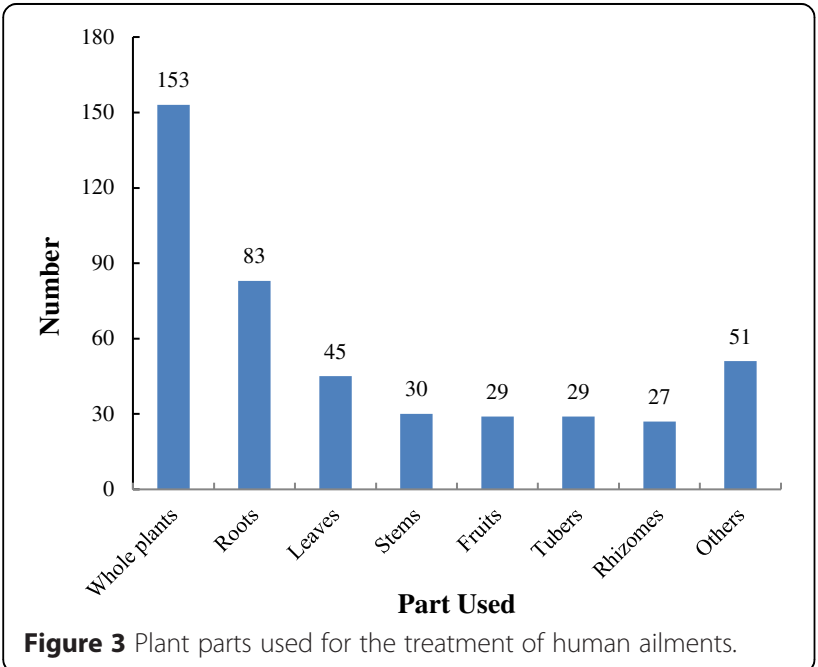

flavor. Most informants used measuring units such as cup, bowl, spoon, fingers and scale but still differed in the doses they administered. The various ways of measuring dosage were generally categorized under three major classes. One dosage was used for those medicinal plants which were expected to be highly toxic. For such medicines the measurement was undertaken by number or weight. The second was the dosage used for medicinal plants which have side effect. The dosage was measured by their hand and taken by container. The third case referred to the medicinal plants without any observable side effects. Medicines prepared were taken according to patients' personal preference.

Most of the medicinal plant preparations involved the use of single plant species or a single plant part while those mixing different plants or plant parts were less encountered in the study area excluding those for treating bone fracture, rheumatism and other difficult diseases. Suffering from common diseases (common cold, indigestion, mosquito bite and so on), the Maonans usually picked up some medicinal plants for treatments by themselves. Otherwise, they should turn to the Maonan healers for help, and the local healers usually prepared remedies by mixing various plants or plant parts. Lack of consistency regarding amount of medicines was observed among informants. There was no concise standard in measurement or unit used among the informants.

\section{Threats to medicinal plants and conservation practices}

Various factors that were considered as main threats for medicinal plants were recorded by discussion with the informants in the study area. The principal threats of medicinal plants were reported to include drought, deforestation, medicinal purpose, and firewood collection in this area. Informants ranked that the major factors were deforestation for the purpose of agricultural expansion (75\%), drought (10\%), collection of medicinal plant material $(10 \%)$ and fire wood (5\%). The Maonan people knew the benefits of conserving medicinal plants. However, the effort of conserving medicinal plants was very limited, because most medicinal plants were collected from wild. Even the local healers who frequently made use of medicinal plants for livelihood did not conserve medicinal plants very well, and they preferred to collect them from wild when using for patients.

\section{Discussion}

Medicinal plants and associated traditional knowledge

On the basis of field investigation and literature studies, 368 species of medicinal plants belonged to 295 genera and 115 families were cataloged. Chinese name, scientific name, local name, family name, used parts and the treatment of diseases were listed. Asteraceae (with 24 species) occupied the highest proportion (6.52\%), followed by 
Table 4 Informant consensus factor by categories of diseases in the study area

\begin{tabular}{|c|c|c|c|c|c|}
\hline Category & Number of spp. & Total of spp. (\%) & Number of use citation & Total of use citations (\%) & $F_{I C}$ \\
\hline $\begin{array}{l}\text { Stomach, intestine and liver diseases } \\
\text { (Internal Organ) }\end{array}$ & 139 & 37.57 & 236 & 10.69 & 0.41 \\
\hline Respiratory system & 83 & 22.43 & 153 & 6.93 & 0.46 \\
\hline Rheumatic problems & 83 & 22.43 & 217 & 9.83 & 0.62 \\
\hline Traumatic injury and sprain & 72 & 19.46 & 275 & 12.46 & 0.74 \\
\hline Skin diseases, skin cut and wound & 65 & 17.57 & 152 & 6.89 & 0.58 \\
\hline Urinary system & 47 & 12.70 & 105 & 4.76 & 0.56 \\
\hline Inflammation & 48 & 12.97 & 143 & 6.48 & 0.67 \\
\hline Infectious diseases & 40 & 10.81 & 78 & 3.53 & 0.49 \\
\hline Fever and malaria & 36 & 9.73 & 132 & 5.98 & 0.73 \\
\hline Bleeding and hemorrhages & 36 & 9.73 & 95 & 4.30 & 0.63 \\
\hline Pain & 30 & 8.11 & 64 & 2.90 & 0.54 \\
\hline $\begin{array}{l}\text { Animal bite (snake, centipede, } \\
\text { mosquito and bat) }\end{array}$ & 30 & 8.11 & 86 & 3.90 & 0.66 \\
\hline Gynecological problems & 29 & 7.84 & 54 & 2. 45 & 0.47 \\
\hline Infantile diseases & 28 & 7.57 & 110 & 4.98 & 0.75 \\
\hline Heart and circulatory system & 25 & 6.76 & 42 & 1.90 & 0.41 \\
\hline Male problems & 25 & 6.76 & 76 & 3.44 & 0.68 \\
\hline Nerves and psychosomatic problems & 12 & 3.24 & 14 & 0.63 & 0.15 \\
\hline Hyperlipidemia and diabetes & 6 & 1.62 & 13 & 0.59 & 0.58 \\
\hline Brain diseases & 5 & 1.35 & 7 & 0.32 & 0.33 \\
\hline Cancer and tumors & 4 & 1.08 & 6 & 0.27 & 0.40 \\
\hline Other Uses (edema, swelling and so on) & 87 & 23.51 & 149 & 6.75 & 0.42 \\
\hline
\end{tabular}

Fabaceae, Rosaceae and Euphorbiaceae. Moerman also found that species of plants in the sunflower family (Asteraceae) tended to be represented in ethnobotanical usage in excess of what would be expected by their occurrence in local environments [27-29]. In contrast, Moerman identified the greater number of families across North America in general. The most widely used plant remedies by the Maonans were obtained from herbaceous species which constituted the highest category of 203 species $(55.16 \%)$. Similar findings were reported by other studies throughout the world, and the authors reported that people derived their medicine from herbs partly because of the fact that forests had been degraded, and it took less time and effort to harvest plant material from medicinal herbs [6,30-32].

The special geographical environment results in the rich biodiversity of medicinal plants in the study area. The Maonans have learnt to use local medicinal plants for treatment and prevention in the course of struggling with the ailments. The number of reported medicinal plants and their uses by the Maonans indicate the depth of indigenous knowledge on the medicinal plants and

Table 5 Preference ranking to medicinal plants used to treat traumatic injury

\begin{tabular}{|c|c|c|c|c|c|c|c|c|c|c|}
\hline \multirow[t]{2}{*}{ List of medicinal plants } & \multicolumn{8}{|c|}{ Informants } & \multirow[t]{2}{*}{ Total } & \multirow[t]{2}{*}{ Rank } \\
\hline & $\mathrm{R}_{1}$ & $\mathbf{R}_{2}$ & $\mathbf{R}_{\mathbf{3}}$ & $\mathbf{R}_{\mathbf{4}}$ & $\mathrm{R}_{5}$ & $\mathbf{R}_{6}$ & $\mathbf{R}_{\mathbf{7}}$ & $\mathbf{R}_{\mathbf{8}}$ & & \\
\hline Acanthopanax trifoliatus & 4 & 7 & 5 & 5 & 7 & 5 & 4 & 5 & 42 & 2 \\
\hline Bauhinia championii & 3 & 4 & 6 & 7 & 1 & 3 & 1 & 2 & 27 & 5 \\
\hline Gaultheria leucocarpa & 5 & 5 & 7 & 6 & 5 & 6 & 6 & 3 & 43 & 1 \\
\hline Justicia ventricosa & 2 & 6 & 3 & 1 & 4 & 4 & 2 & 4 & 26 & 6 \\
\hline Polygonum chinense & 6 & 1 & 1 & 2 & 2 & 1 & 3 & 1 & 17 & 7 \\
\hline Sargentodoxa cuneata & 7 & 3 & 4 & 4 & 3 & 7 & 5 & 7 & 40 & 3 \\
\hline Sambucus williamsii & 1 & 2 & 2 & 3 & 6 & 2 & 7 & 6 & 29 & 4 \\
\hline
\end{tabular}

Key-R represented respondents; Scores in the table indicated ranks given to medicinal plants based on their scarcity. Highest number (7) is for the medicinal plants which informants thought most preferred in the area and the lowest number (1) for the least preferred medicinal plant. 
Table 6 Most frequently inventoried medicinal plants

\begin{tabular}{lllll}
\hline Species name & $\begin{array}{l}\text { Medicinal } \\
\text { value }\end{array}$ & $\begin{array}{l}\text { Edible } \\
\text { value }\end{array}$ & $\begin{array}{l}\text { Economic } \\
\text { value }\end{array}$ & $\begin{array}{l}\text { Ornamental } \\
\text { value }\end{array}$ \\
\hline Acanthopanax trifoliatus & $\sqrt{ }$ & $\sqrt{ }$ & $\sqrt{ }$ & $\sqrt{ }$ \\
Buddleja officinalis & $\sqrt{ }$ & $\sqrt{ }$ & $\sqrt{ }$ & \\
Houttuynia cordata & $\sqrt{ }$ & $\sqrt{ }$ & $\sqrt{ }$ & \\
Litsea pungens & $\sqrt{ }$ & $\sqrt{ }$ & $\sqrt{ }$ & $\sqrt{ }$ \\
Murraya exotica & $\sqrt{ }$ & & $\sqrt{ }$ & $\sqrt{ }$ \\
Nephrolepis cordifolia & $\sqrt{ }$ & & $\sqrt{ }$ & \\
Paederia scandens & $\sqrt{ }$ & $\sqrt{ }$ & & \\
Platycodon grandiflorus & $\sqrt{ }$ & $\sqrt{ }$ & $\sqrt{ }$ & $\sqrt{ }$ \\
Rauvolfia verticillata & $\sqrt{ }$ & & $\sqrt{ }$ & $\sqrt{ }$ \\
Rubus parvifolius & $\sqrt{ }$ & $\sqrt{ }$ & $\sqrt{ }$ & $\sqrt{ }$ \\
Sargentodoxa cuneata & $\sqrt{ }$ & & $\sqrt{ }$ & \\
Talinum paniculatum & $\sqrt{ }$ & $\sqrt{ }$ & $\sqrt{ }$ & $\sqrt{ }$ \\
Tetrapanax papyrifer & $\sqrt{ }$ & & $\sqrt{ }$ & \\
\hline
\end{tabular}

their applications. The Maonans have collected their indigenous knowledge and experience of medicinal plants. Without written language, the knowledge of medicinal plants is still taught orally in the Maonan communities. There is not data record or any illustrated identification which guides for the medicinal plants of Maonan people and their uses.

The Maonans have the traditional customs of disease prevention and emphasize on the function of medicinal food in ordinary life. They usually add medicinal plants into food for the purpose of enhancing the body's immunity and disease resistance, such as Talinum paniculatum, Gymnotheca chinensis, Osbeckia opipara and so on. The Maonans have the custom of collecting the medicinal plants for cooking and bathing in dragon-boat festival, such as Acorus calamus, Curcuma longa, Paederia scandens and Leonurus artemisia. They believe that it would be beneficial for their health. This is because many plants matured in the season of dragonboat festival [33].

\section{Preparation, dosage and route of administration of medicinal plants}

The most widely harvested part was the whole plant, followed by the roots, leaves, stems and others. The Maonan people used a lot of roots, stems, rhizomes and bark for medicinal purpose. They believed these parts were the most effective. However, such collection of the medicinal plants might kill or damage plants when harvesting. Utilization of leaves might not cause detrimental effect on the plants compared with plant species that root was utilized. Most of medicinal plants were claimed to be prepared from a single species or plant part in the present study, and the different parts of medicinal plant were used to treat disparate diseases. Although Maonan people preferred to treat illnesses with single species, it was observed that the healers mostly used multiple species or plant parts in order to increase the function and efficacy of the drug as they reported during the interviews. Representatively, the Maonan healers mostly used more than one plant species to prepare remedy for treating bone fracture and traumatic injury.

Grinding was the most widely used method of preparation for remedy in the study area. Pounding and powdering fresh plant materials were the other methods of preparation in the study area. Due to the efficiency and richness of the fresh medicinal plants in the study area, preference of application of fresh plant parts was observed. Moreover, internal and skin diseases were more prevalent in the study area. The fresh material use might be an attempt not to lose volatile oils, the concentration of which could decrease on drying. Moa et al. reported that the disadvantage was that utilization of fresh plant parts may threaten the plants through frequent collection including in dry seasons since local people made minimal efforts in storing dried plant material for later use [6].

The Maonans usually use the processing methods such as decoction, medicinal liquor, external application and medicated bath. The way of using herbs was benefit for the popularity in a simple and easy method. They used different additives like alcohol, oil, honey, salt, sugar, eggs, chicken, duck and meat in order to increase the flavor, taste and general acceptability of certain orally administered remedies. Because of poverty, eating animal meat and eggs could increase proteins and might be helpful for body recovery when the Maonans were ill. The Maonan healers considered that alcohol could promote the blood circulation and accelerate the absorption of exudates. In addition, the Maonan healers used different procedures to administer the medicinal plants and alcohol combinations. The medicinal plants were soaked in alcohol for nearly one month and then the patients could drink or applied externally on the affected parts. For example, Acanthopanax gracilistylus, Achyranthes bidentata, Ardisia gigantifolia, Ardisia japonica, Arisaema heterophyllum, Davallia mariesii, Dipsacus asperoides, Drynaria propinqua, Homalomena occulta, Sambucus williamsii, Bauhinia championii, Murraya exotica, and Paris polyphylla were usually soaked in alcohol for treating traumatic injury and bone fracture.

\section{Effectiveness and popularity of medicinal plants}

Due to the influence of geography, climate and food culture in Maonan areas, the Maonan healers understood the varieties of diseases, such as traumatic injury, snake bite, hepatitis, respiratory disease, digestive system disease, rheumatoid arthritis, and skin problems. The local people expressed they preferred to use 
traditional medicines rather than western drugs to get relief from some diseases including bone fracture, health problems associated with the liver, snake bite and those caused by hepatitis. The Maonan healers treated ailments based on the patients' physical conditions, lack of consistency regarding amount of medicines to be used was observed among informants during the interviews. The healers usually did not know which ingredients were important for the therapeutic effect in the multiple prescriptions. The lack of precise dosage was one shortage of traditional medicinal plant uses.

Most of Maonan people knew how to use several medicinal plants for treating ailments and health protection. Traditional medicine knowledge was not only in the hands of the Maonan healers and herbalists in the study area. Moreover, Maonan people grew medicinal plants in their home gardens. Plant species maintained by Maonan healers was found to be significantly distinct from plant species managed by farmers. The Maonan healers knew more than 30 medicinal plant species, while most of the non-healers reported less than 15 species. Ethnomedicinal usage of plants managed by healers was remarkably distinct from usage categories managed by farmers. The Maonan healers were reported to use a combination of multiple medicinal plants to treat an illness, but the farmers always used single plant species or a single plant part.

\section{Medicinal plant cultivation and trade}

The Maonan people in the study area knew the benefits of conserving medicinal plants. However, the effort of conserving medicinal plants was very limited. For example, only $20.75 \%$ of medicinal plants were collected from home gardens, and most of the plants from home gardens were used for foods, spices and substitutes for tea. The majority of medicinal plants were harvested from wild habitats. Even Maonan healers who made use of medicinal plants for a livelihood did not conserve the important medicinal plants in their home gardens, and they preferred to collect them from wild or hidden places when patients visited them. It was explained by informants that local healers did not let the other villagers know the identity of the medicinal plants they were using. Informants further explained that if healers planted the species in their home gardens, they worried that somebody else might recognize them when they were preparing the medicine from the plants. Thus the healers' income would be decreased.

Because of complex terrain and language barrier, the Maonans have been in the traditional self-sufficient agricultural economy in the karst areas. There is a seasonal medicinal market which opens 3 times each month. The sites of purchase and sale of local medicinal plants are located in the town. The medicinal plants grown by farmers were used for household healthcare and little was sold in herbal markets, while medicinal plants were cultivated by healers rarely for trading, either. Not many medicinal plants were solely cultivated for their medicinal purpose, except that the plants were multipurpose (Table 6). Lack of water and land, most Maonan people would prefer to cultivate foods or cash crops rather than medicinal plants. The other reason was that most medicinal plants were not sold at reasonable prices and therefore not profitable, providing very little incentives for their cultivation. The local medicinal markets were small-scaled and were not paid enough attention. The markets provided convenience for the exchange of local medicinal plants, but not providing a good place for indigenous knowledge. This trend might not be beneficial for maintaining traditional practices and giving traditional knowledge the respect it deserves.

\section{Threats to medicinal plants and conservation practices in the study area}

According to informants, nowadays it would take a lot of time and travel long distances to search for some medicinal plants, especially trees and some shrubs. The principal threats of medicinal plants were reported in the study area, including deforestation for agricultural purposes, urbanization, drought, over-harvesting of known medicinal species and firewood collection. Also, informants ranked deforestation for agricultural purposes as the most serious threat to medicinal plants followed by drought, collection of other different factors and firewood. The conservation of medicinal plants was less realized in the study area.

\section{Medicinal plants knowledge secrecy, mode of transfer, gender issue and threats between different social groups within the Maonan area}

This study highlighted the rich biodiversity of medicinal plants and ethnomedicinal practice in Maonan area to maintain wellbeing and support livelihoods. This study revealed that, most of the knowledge on herbal remedies was handled down to the younger members of the community by elders orally, who were over 40 years old and lesseducated. The Maonan herbalists and healers were male, and only men had the opportunities to study knowledge of traditional medicinal plants in the family. The conservative concept of Maonan healers made a systematic indigenous knowledge of Maonan traditional medicine, which had always been in the hands of a few people. The age structure and knowledge transmission system had the negative influence on the inheritance and development of indigenous knowledge. It dramatically exposed the vulnerability of traditional medicinal knowledge if its transmission was limited by acculturation or inter-ethnic exchange from generation to generation $[15,34,35]$. 
Nowadays, the fact is that inheritance of indigenous knowledge is difficulty from the elders to the young generation. Most young people do not believe that studying indigenous knowledge is beneficial for their life because it is less profited compared to working in the urban area. Furthermore, some young people think traditional medicine is anti-science. While male Maonan people work outside, women take responsibility to take care of their families and educate children. If women know how to use medicinal plants, it will be beneficial for training children. According to our interviews, the Maonan women are eager to learn the traditional herbal medicinal knowledge. They may become potential and effective inheritors in the Maonan area, if customary inheriting system allows them.

\section{Conclusions}

The paper is an ethnobotanical study on medicinal plants used by Maonan people. We documented 368 species (belonging to 295 genera and 115 families) of medicinal plants used by the Maonans in Huanjiang Maonan Autonomous County, northern Guangxi, southwest China. These plants were used to treat 95 human diseases, such as traumatic injury, bone fracture, health problems associated with the liver disorder, snake bite, and spider poisoning etc. Traditional knowledge about the use, preparation, and application of these medicinal plants is usually passed verbally from generation to generation. The valuable information about medicinal plants could be preserved while recording in the written form. Moreover, the documentation of medicinal plants can serve as a basis for future investigation of new medicinal resources.

Among the medicinal plant species, the whole plants of herbaceous species are harvested from field and constituted the highest proportion of medicinal plants to be utilized. More roots and barks are used than other plant parts, which imply that traditional medical culture in the Maonan area does threaten some species. Although high numbers of medicinal plant species have been reported to be used for human health problems, many wild species are being threatened by various anthropogenic factors while conservation efforts are less practiced in the study area. Deforestation for agricultural purposes is the major threat factor. To save and protect medicinal plants, the external help is necessary, by providing the Maonan people with both seedlings or seeds and cultivation techniques of medicinal plants.

The Maonan men are the only inheritors to transmit traditional medicinal knowledge to the next generations. Unfortunately, the knowledge on herbal remedies is held by elders, who are less educated and above 40 years old. Most young men prefer to look for jobs in urban areas instead of studying the Maonan's medicinal knowledge. It is urgent to find solution of conserving and transmitting the traditional medicinal knowledge in the study area.
Thus, government agencies should encourage the Maonan people to maintain the biodiversity and the ethnomedicinal knowledge by providing the local people with planting materials of the most threatened and preferred medicinal and multipurpose species so that they can grow these plants in their home gardens or farmlands. Public awareness is needed to encourage the local Maonan people to sustainably utilize and manage the medicinal plant resources. Ex situ and in situ conservation measures should be taken to protect the medicinal plants in the study areas from further loss.

\section{Consent}

Permissions were provided by all participants in this study, including the local Maonan people. Consent was obtained from the local communities prior to the field investigations. The authors have all copyrights.

\section{Competing interests}

The authors declare that they have no competing interests.

\section{Authors' contributions}

LYH designed the study, analyzed the data, and drafted the manuscript. SWM contributed to the preparation of the manuscript. CLL designed the study, revised and finalized the manuscript. All authors participated in the field surveys. All authors read and approved the final manuscript.

\section{Acknowledgements}

We are grateful to the administrative agencies of Huanjiang Maonan Autonomous County for their help while carrying out this study. We fully acknowledged the local informants and healers for participating the surveys and sharing their knowledge on the use of medicinal plants with us. Without their contribution, this study would have been impossible. We would like to thank Qingqing Bi from the Department of Minority Languages and Literatures at Minzu University of China for correcting and typing the Maonan names of medicinal plants. This work was financially supported by the National Natural Science Foundation of China (31161140345, 31070288), Minzu University of China (10301-01404031-84, YLDX01013), Ministry of Science and Technology of China (2012FY110300), Ministry of Education of China and State Administration of Foreign Experts Affairs of China (B08044).

\section{Author details}

${ }^{1}$ College of Life and Environmental Sciences, Minzu University of China, Beijing 100081, People's Republic of China. 'Lineberger Comprehensive Cancer Center, University of North Carolina at Chapel Hill, Chapel Hill, NC 27599, USA. ${ }^{3}$ Kunming Institute of Botany, Chinese Academy of Sciences, Kunming 650201, People's Republic of China.

Received: 29 November 2014 Accepted: 11 April 2015

Published online: 30 April 2015

\section{References}

1. Huai HY, Pei SJ. Medicinal ethnobotany and its advances. Chin Bull Bot. 2002;2(19):129-36.

2. Zhu YP, Woerdenbad HJ. Traditional Chinese herbal medicine. Pharm World Sci. 2005;4(17):103-12. doi:10.1007/BF01872386.

3. Pei SJ. Overview of medicinal plants and its conservation in China. J Xinjiang Univ (Nat Sci Ed). 2007;24:317-22.

4. Farnsworth NR. Ethnopharmacology and future dug development: the North American experience. J Ethnopharmcol. 1993;38:145-52. doi:10.1016/0378-8741(93)90009-T.

5. Samy RP, Gopalakrishnakone P. Current status of herbal and their future perspectives. Nat Proc. 2007;1176:1-13. hdl: 10101/npre.2007.1176.1: Posted 28 Sep 2007

6. Moa M, Zemede A, Ensermu K, Abebe B, Bizuneh W. An ethnobotanical study of medicinal plants in Wayu Tuka District, East Welega Zone of 
Oromia Regional State, West Ethiopia. J Ethnobiol Ethnomed. 2013;9:68. doi:10.1186/1746-4269-9-68.

7. Bussmann RW, Sharon D. Traditional medicinal plant use in Northern Peru: tracking two thousand years of healing culture. J Ethnobiol Ethnomed. 2006;2:47. doi:10.1186/1746-4269-2-47.

8. MacDonald I. Current trends in ethnobotany. Trop J Pharm Res. 2009;8(4):295-6.

9. Begossi A, Hanazaki N, Tamashiro JY. Medicinal plants in the Atlantic forest (Brazil): knowledge, use, and conservation. Hum Ecol. 2002;30:281-99. doi:10.1023/A:1016564217719.

10. Frei B, Sticher O, Heinrich M. Zapotec and Mixe use of tropical habitats for securing medicinal plants in Mexico. Econ Bot. 2000;54:73-8. doi:10.1007/BF02866601.

11. Thomas E, Vandebroek I, van Damme P. Valuation of different forests and plant species in indigenous territory and national park Isiboro-Secure Bolivia. Econ Bot. 2009:63:229-41. doi:10.1007/s12231-009-9084-5.

12. Liu YJ, Ahmed S, Liu B, Guo ZY, Huang WJ, Wu XJ, et al. Ethnobotany of dye plants in Dong communities of China. J Ethnobiol Ethnomed. 2014:10:23. doi:10.1186/1746-4269-10-23

13. Pei SJ. Brief discussion on ethno-medicine research and new-drug development of China. J Yunnan Coll Tradit Chin Med. 2007;30(3):1-7.

14. Ana LC, Cadena G, Marten S, Ida T. Use and valuation of native and introduced medicinal plant species in Campo Hermoso and Zetaquira, Boyacá Colombia. J Ethnobiol Ethnomed. 2013;9:23. doi:10.1186/1746-4269-9-23.

15. Muthu C, Ayyanar M, Rajan N, Ignacimuthu S. Medicinal plants used by traditional healers in Kaancheepuram District of Tamil Nadu India. J Ethnobiol Ethnomed. 2006;2:43. doi:10.1186/1746-4269-2-43.

16. Yang LX, Ahmed S, Stepp JR, Mi K, Zhao YQ, Ma JZ, et al. Comparative homegarden medical ethnobotany of Naxi healers and farmers in Northwestern Yunnan China. J Ethnobiol Ethnomed. 2014;10:6. doi:10.1186/1746-4269-10-6.

17. Huang J, Pei SJ, Long CL. An ethnobotanical study of medicinal plants used by the Lisu people in Nujiang, Northwest Yunnan China. Econ Bot. 2004;58(1):S253-64. doi:10.1663/0013-0001(2004)58[S253:AESOMP]2.0.CO;2.

18. Liu YC, Dao ZL, Yang CY, Liu YT, Long CL. Medicinal plants used by Tibetans in Shangri-la, Yunnan China. J Ethnobiol Ethnomed. 2009;5:15. doi:10.1186/ 1746-4269-10-23.

19. Meyer F. Theory and Practice of Tibetan Medicine. In: Alphen JV, Aris A, editors. Oriental Medicine: An Illustrated Guide to the Asian Arts of Healing. Chicago: Serindia Publications; 1995. p. 109-43. republished by Shambala Publ., Boston, 1997.

20. Ma L, Gu RH, Tang L, Chen ZE, Di R, Long CL. Important poisonous plants in Tibetan ethnomedicine. Toxins. 2015;7(1):138-55. doi:10.3390/toxins7010138.

21. Li SM, Long CL, Liu FY, Lee S, Guo Q, Li R, et al. Herbs for medicinal baths among the traditional Yao communities of China. J Ethnopharmacol. 2006;108(1):59-67. doi:10.1016/j.jep.2006.04.014.

22. Lu TQ. A Grammar of Maonan. Boca Raton, Florida: Universal Publishers; 2008.

23. Martin GJ. Ethnobotany: A Method Manual. London: Chapman and Hall; 1995

24. Teklay A, Abera B, Giday M. An ethnobotanical study of medicinal plants used in Kilte Awulaelo District Tigray Region of Ethiopia. J Ethnobiol Ethnomed. 2013;9:65. doi:10.1186/1746-4269-9-65.

25. Heinrich M, Ankli A, Frei B, Weimann C, Sticher O. Medicinal plants in Mexico: Healers' consensus and cultural importance. Soci Sci Med. 1998:47:1863-75. doi:10.1016/S0277-9536(98)00181-6.

26. Heinrich M. Ethnobotany and its role in drug development. Phytother Res. 2000;14:479-88.

27. Moerman DE. Symbols and selectivity: A statistical analysis of native American medicinal ethnobotany. J Ethnopharmacol. 1979;1:111. doi:10.1016/0378-8741(79)90002-3.

28. Moerman DE. Poisoned apples and honeysuckles: The medicinal plants of native America. Med Anthropol Q. 1989;3:52-61. doi:10.1525/ maq.1989.3.1.02a00040.

29. Moerman DE. Native American Ethnobotany. Portland, Oregan: Timber Press; 1998. 927 pp.

30. Giday M. An ethnobotanical study of medicinal plants used by the Zay people in Ethiopia. Skriftserie. 2001;3:81-99. doi:10.1016/S0378-8741(02)00359-8.

31. Stepp JR, Moerman DE. The importance of weeds in ethnopharmacology. J Ethnopharmacol. 2001;75(1):19-23. doi:10.1016/S0378-8741(00)00385-8.

32. Stepp JR. The role of weeds as sources of pharmaceuticals. J Ethnopharmacol. 2004;92(2):163-6. doi:10.1016/j.jep.2004.03.002.
33. Yang CY, Long CL, Shi YN, Wang YH, Wang HS. Ethnobotaniacal study on medicinal market during Dragon-boat Festival in Jingxi County, southwestern Guangxi region. J CUN (Nat Sci Ed). 2009;18(2):16-26.

34. Singh GA, Kumar A, Tewari DD. An ethnobotanical survey of medicinal plants used in Terai forest of western Nepal. J Ethnobiol Ethnomed. 2012;8:19. doi:10.1186/1746-4269-8-19.

35. Bekalo HT, Woodmatas DS, Woldemariam AZ. An ethnobotanical study of medicinal plants used by local people in the lowlands of Konta Special Woreda, southern nations, nationalities and peoples regional state Ethiopia. J Ethnobiol Ethnomed. 2009;5:26. doi:10.1186/1746-4269-5-26.

\section{Submit your next manuscript to BioMed Central and take full advantage of:}

- Convenient online submission

- Thorough peer review

- No space constraints or color figure charges

- Immediate publication on acceptance

- Inclusion in PubMed, CAS, Scopus and Google Scholar

- Research which is freely available for redistribution

Submit your manuscript at www.biomedcentral.com/submit
C BioMed Central 